Portland State University

PDXScholar

Spring 1-1-2010

\title{
Characterization of Mesoscopic Fluid-like Films with the Novel Shear-force/Acoustic Microscopy
}

Xiaohua Wang

Portland State University

Follow this and additional works at: https://pdxscholar.library.pdx.edu/open_access_etds Let us know how access to this document benefits you.

\section{Recommended Citation}

Wang, Xiaohua, "Characterization of Mesoscopic Fluid-like Films with the Novel Shear-force/Acoustic Microscopy" (2010). Dissertations and Theses. Paper 736.

https://doi.org/10.15760/etd.736

This Thesis is brought to you for free and open access. It has been accepted for inclusion in Dissertations and Theses by an authorized administrator of PDXScholar. Please contact us if we can make this document more accessible: pdxscholar@pdx.edu. 
Characterization of Mesoscopic Fluid-like Films with

the Novel Shear-force/Acoustic Microscopy

by

Xiaohua Wang

A thesis submitted in partial fulfillment of the requirements for the degree of

Master of Science

in

Physics

Thesis Committee:

Andres H. La Rosa, Chair

Raj Solanki

Mingdi Yan

Portland State University

(C) 2010 


\begin{abstract}
The shear force mechanism has been utilized as a distance regulation method in scanning probe microscopes. However, the origin of shear force is still unclear. One of the most important reasons for the shear-force damping is due to the presence of a water contamination layer at the sample surface in ambient conditions. Understanding the behavior of such mesoscopic fluid-like films is of significance for studies of not only scanning probe microscopy but also other complex surface phenomena, such as nanotribology, lubrication, adhesion, wetting, and the microfluidity of biological membranes. This thesis investigates, in particular, the dynamics of mesoscopic fluids confined between two sliding solid boundaries. When fluids are constrained to nanometer-sized regions, their physical properties can greatly differ from those displayed by bulk liquids. To gain an insight into the fundamental characteristics of the confined fluid films, we exploit the versatile capabilities of the novel shear-force/acoustic nearfield microscope (SANM), which is able to concurrently and independently monitor the effects of the fluid-mediated interactions acting on both the microscope's probe and the sample to be analyzed. Two signals are monitored simultaneously during each experimental cycle: the tuning fork signal, which is the oscillation amplitude of the probe and gives access to the shear force; and acoustic signal, which is detected by an acoustic sensor placed under the sample. Systematic experiments are carried out to investigate the effects of probe geometry, environmental humidity, and chemical properties of probe and sample surface (water affinity: hydrophobicity or hydrophilicity) on the probe-sample interactions, expressing the influence of the fluid-like contamination films.
\end{abstract}




\section{ACKNOWLEDGEMENTS}

I wish to express my warm and sincere thanks to my advisor Prof. Andres H. La Rosa. His wide knowledge and logical way of thinking have been of great value for me. His understanding, encouraging and personal guidance have provided a good basis for the present thesis.

I would like thank my labmates: Rodolfo Fernandez, Keith Parker, and others. It is a pleasure to work with you guys, and discuss about our research. All of you offered me lots of helps, as well as valuable suggestions to my works. I would like to thank Marc Nisenfeld for all of mechanical fabrications.

I also want to thank Prof. Mingdi Yan from Department of Chemistry and people

from her lab: Suji Uppalapati, Sailaja Chada, Dr. Lihong Liu, and Dr. Hui Wang. Without their kind efforts on sample preparation and generous help in chemistry, I cannot have chance to work on my projects.

I owe my loving thanks to my fiancé Xin Wang, currently a $\mathrm{PhD}$ candidate in Chemistry from Dr Yan's Group. Without his encouragement and great helps it would have been impossible for me to finish this work. 


\section{TABLE OF CONTENTS}

ACKNOWLEDGEMENTS

LIST OF FIGURES $\quad$ v

\section{CHAPTER}

1. INTRODUCTION $\ldots \ldots \ldots \ldots \ldots \ldots \ldots \ldots \ldots \ldots \ldots \ldots \ldots \ldots \ldots$

1.1 Motivations of the thesis $\ldots \ldots \ldots \ldots \ldots \ldots \ldots \ldots \ldots \ldots$

1.2 Overview of scanning probe microscopy $\ldots \ldots \ldots \ldots \ldots \ldots \ldots$

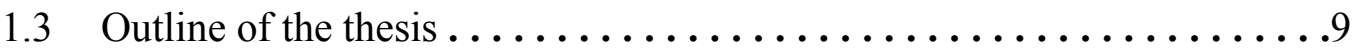

2. THEORETICAL STUDIES UNDERLYING THE EXPERIMENTS . . . 11

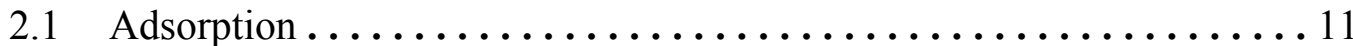

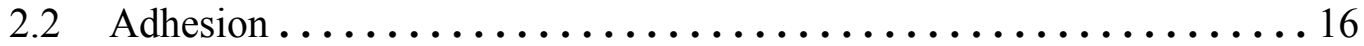

2.3 Effect of capillary condensation $\ldots \ldots \ldots \ldots \ldots \ldots \ldots \ldots \ldots \ldots \ldots \ldots \ldots \ldots$

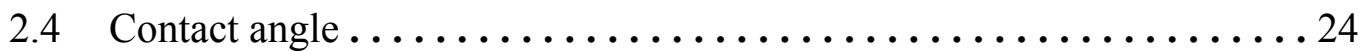

2.5 Hydrophobic/hydrophilic interactions .................... 26

3. FABRICATION OF METALLIC PROBES AND EXPERIMENTAL

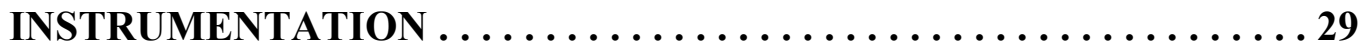

3.1 Fabrication of tapered gold tips . . . . . . . . . . . . . . . 29

3.2 Experimental instrumentation $\ldots \ldots \ldots \ldots \ldots \ldots \ldots \ldots \ldots \ldots$

4. INVESTIGATION OF INTERACTIONS BETWEEN DIFFERENT PROBES AND CHEMICALLY TREATED SAMPLES BASED ON SHEAR-FORCE/ACOUSTIC MEASUREMENTS . . . . . . . . . . .42

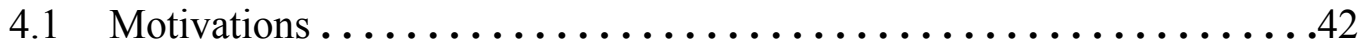

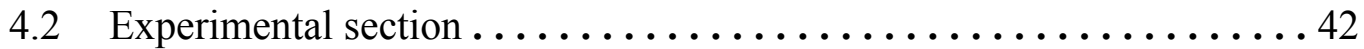

4.3 Results and discussions $\ldots \ldots \ldots \ldots \ldots \ldots \ldots \ldots \ldots \ldots \ldots$ 
4.4 Conclusions ...............................65

5. CONTACT CURRENT: THE INDICATOR OF SOLID-SOLID TIPSAMPLE MECHANICAL CONTACT ...............67

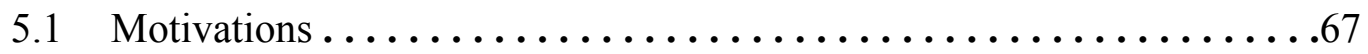

5.2 Experimental section $\ldots \ldots \ldots \ldots \ldots \ldots \ldots \ldots \ldots \ldots \ldots \ldots \ldots \ldots \ldots \ldots$

5.3 Results and discussions $\ldots \ldots \ldots \ldots \ldots \ldots \ldots \ldots \ldots$

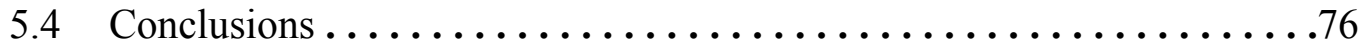

6. ELECTRIC FIELD-ASSISTED DIP-PEN NANOLITHOGRAPHY ON P4VP THIN FILMS ...................... 78

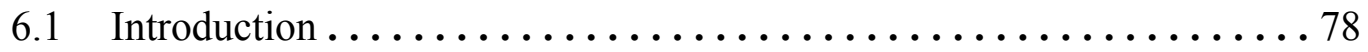

6.2 Experimental section $\ldots \ldots \ldots \ldots \ldots \ldots \ldots \ldots \ldots \ldots \ldots$

6.3 Results and discussions $\ldots \ldots \ldots \ldots \ldots \ldots \ldots \ldots \ldots \ldots \ldots$

6.4 Conclusions ............................ 89

7. FINAL CONCLUSIONS ......................90

8. FUTURE RECOMMENDATION . . . . . . . . . . . . . .92

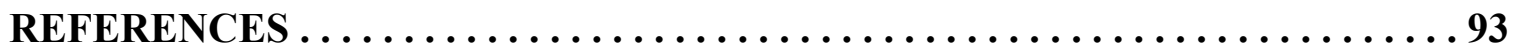




\section{LIST OF FIGURES}

Fig.1. 2.1 The principle of the scanning tunneling microscope as sketched by Binning and Rohrer.......................................4

Fig. 1.2.2 The principle of the atomic force microscope as sketched by Bhushan $\ldots . . .7$

Fig. 2.2.1 Definitions of the works of adhesion and cohesion $\ldots \ldots \ldots \ldots \ldots \ldots 17$

Fig. 2.2.2 (a) Elastic sphere on rigid surface in the absence (Hertz) and presence (JKR) of adhesion. (b) Elastic adhering sphere about to separate spontaneously from

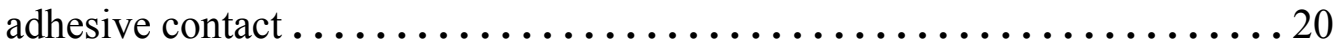

Fig. 2.3 A non-deforming sphere on a rigid flat surface: (a) In a vapor the capillary force arises from the Laplace pressure. (b) In the absence of condensing vapor, the adhesion force is due to the direct contact of the surfaces $\ldots \ldots \ldots \ldots \ldots \ldots .23$

Fig. 2.4 (a) Schematic of a droplet on a solid surface and the definition of the contact angle. (b-d) The various degrees of wetting: complete wetting, partial wetting,

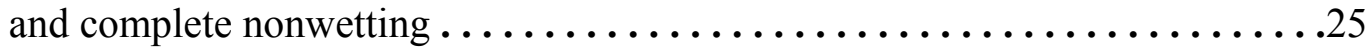

Fig. 3.1.1 Experimental setup used for electrochemically etching gold tips ........ 30

Fig. 3.1.2 Current intensity monitored during different etching processes: (left) etching of gold wire; (right) etching of tungsten wire $\ldots \ldots \ldots \ldots \ldots \ldots \ldots \ldots . . .32$

Fig. 3.1.3 Plots of current intensity vs. time for the processes of etching $0.076 \mathrm{~mm}$ gold wires with different immersion depths, and corresponding optical images of

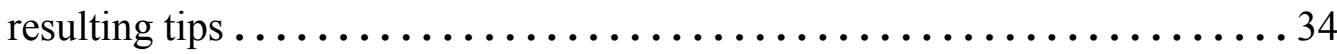

Fig. 3.1.4 Plots of current intensity vs. time for the processes of etching $0.100 \mathrm{~mm}$ gold wires with different immersion depths, and corresponding optical images of

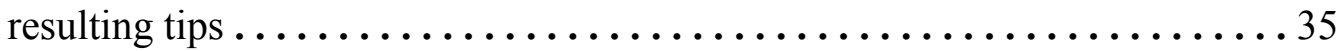


Fig. 3.2.1 A typical probe implemented in our SPM system, which consists of a quartz tuning fork and a tapered gold tip attached to one of its tines $\ldots \ldots \ldots \ldots 37$

Fig. 3.2.2 TF-based probe-sample distance control $\ldots \ldots \ldots \ldots \ldots \ldots \ldots \ldots \ldots$

Fig. 3.2.3 The equivalent circuit of the electrical terminal of $\mathrm{TF} \ldots \ldots \ldots \ldots \ldots \ldots 39$

Fig. 3.2 .4 The microscope stage $\ldots \ldots \ldots \ldots \ldots \ldots \ldots \ldots \ldots \ldots \ldots \ldots \ldots \ldots$

Fig. 4.2 Schematic of the settings used for detecting the the tuning fork (TF) signal along with the acoustic signal as the gold tip approaches to and withdraws from the sample surface $\ldots \ldots \ldots \ldots \ldots \ldots \ldots \ldots \ldots \ldots \ldots \ldots \ldots 44$

Fig. 4.3.1 Scanning electronic images of (a) a "chubby" gold tip and (b) a "sharp" gold

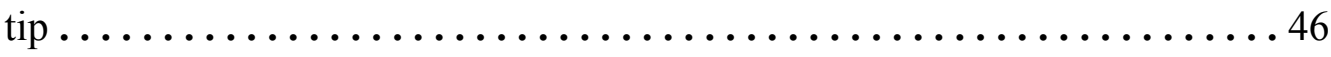

Fig. 4.3.2 Approach and retraction curves of (a) TF and (b) acoustic signals obtained using the chubby tip (humidity: $25 \%$ ) .................. 48

Fig. 4.3.3 Dependences of simultaneously measured TF damping and the acoustic response on the tip-sample distance during the approach process of the chubby

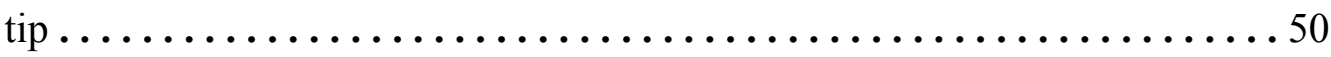

Fig. 4.3.4 Dependences of simultaneously measured TF damping and the acoustic response on the tip-sample distance during the retraction process of the chubby

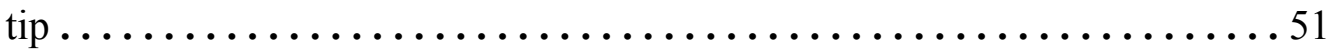

Fig. 4.3.5 Approach and retraction curves of (a) TF and (b) acoustic signals obtained using the sharp tip (humidity: $25 \%$ ) ...................... 52

Fig. 4.3.6 Dependences of simultaneously measured TF damping and the acoustic response on the tip-sample distance during the approach process of the sharp

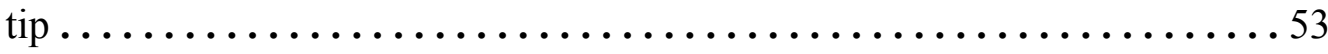


Fig. 4.3.7 Dependences of simultaneously measured TF damping and the acoustic response on the tip-sample distance during the retraction process of the sharp

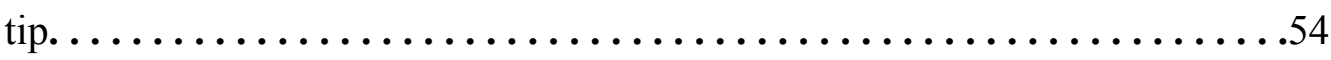

Fig. 4.3.8 Approach and retraction curves of (a) TF and (b) acoustic signals obtained using the chubby tip (humidity: $55 \%$ ) .................. 55

Fig. 4.3.9 Approach and retraction curves of (a) TF and (b) acoustic signals obtained using the sharp tip (humidity: $55 \%) \ldots \ldots \ldots \ldots \ldots \ldots \ldots \ldots$

Fig. 4.3.10 Scheme of the experimental procedure for preparing hydrophobic and hydrophilic surfaces on the same silicon substrate $\ldots \ldots \ldots \ldots . \ldots . \ldots 59$

Fig. 4.3.11 Results of contact angle measurements of the silicon substrate covered by PS and PEOX monolayers, respectively $\ldots \ldots \ldots \ldots \ldots \ldots \ldots \ldots$

Fig. 4.3.12 Approach and retraction curves of (a) TF and (b) acoustic signals obtained when the PS coated gold tip (hydrophobic) approaching to and retracting from the surface covered by PS monolayer (hydrophobic) $\ldots \ldots \ldots \ldots \ldots \ldots 6$

Fig. 4.3.13 Approach and retraction curves of (a) TF and (b) acoustic signals obtained when the PS coated gold tip (hydrophobic) approaching to and retracting from the surface covered by PEOX monolayer (hydrophilic) ...........63

Fig. 4.3.14 The description for an entire approach and retraction cycle obtained using the

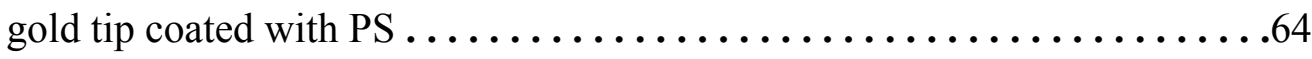

Fig. 4.3.15 Upper: TF (gray) and acoustic (blue) signals obtained when the PEOX-coated tip approaches (a) and retracts from (a') the hydrophilic surface. Lower: TF (gray) and acoustic (green) signals obtained by binging the same tip towards (b) and away from (b') the hydrophobic surface $\ldots \ldots \ldots \ldots \ldots$ 
Fig. 5.2 Schematic of the settings used for detecting the contact current, along with the tuning fork $(\mathrm{TF})$ and the acoustic signals $\ldots \ldots \ldots \ldots \ldots \ldots \ldots \ldots$

Fig. 5.3.1 The spectrum of the tuning fork oscillating in air obtained by performing the frequency sweep from $30200 \mathrm{~Hz}$ to $30800 \mathrm{~Hz}$. . . . . . . . . . . . . . 70

Fig. 5.3.2 The approach and retraction curves of TF and acoustic signals simultaneously recorder by the lock-in amplifiers $\ldots \ldots \ldots \ldots \ldots \ldots \ldots \ldots \ldots \ldots$

Fig. 5.3.3 Simultaneously measured TF damping, the acoustic response and "contact" current dependences on the tip-sample distance during the approach . . . . . 74

Fig. 5.3.4 Simultaneously measured TF damping, the acoustic response and "contact" current dependences on the tip-sample distance during retraction . . . . . . . 74

Fig. 5.3.5 Approach curves of TF, acoustic and current signals obtained by repeating the approach/retraction experiment at a different location $\ldots \ldots \ldots \ldots \ldots \ldots 75$

Fig. 5.3.6 Retraction curves of TF, acoustic and current signals obtained by repeating the approach/retraction experiment at a different location $\ldots \ldots \ldots \ldots \ldots \ldots 76$

Fig. 6.3.1 Different experimental settings used for patterning features on P4VP polymer films: A tip was inked with the acidic buffer and (a) not biased, (b) negatively biased, (c) positively biased. (d) An uncoated tip while applying a positive

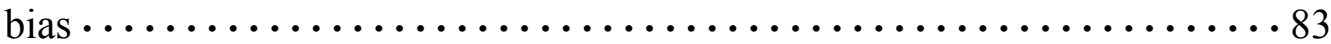

Fig. 6.3.2 (a-e) Topographic images of features drawn using the tip coated with acidic ink. The applied bias voltages $(\mathrm{V})$, contact forces $(\mu \mathrm{N})$ and measured heights of selected lines (nm) are the following: (a) 5, 1, 13; (b) 3, 1, 6; (c) 0, 1, 3; (d) 5, $0.8,8$; (e) 5, 0.5, 3. (f) A line feature with $60 \mathrm{~nm}$ wide. (g) A pattern of the letters: PSU. Image sizes: $8 \mu \mathrm{m} \times 8 \mu \mathrm{m}$ for (a-e); $5 \mu \mathrm{m} \times 5 \mu \mathrm{m}$ for (f-g). (h) 
Schematic showing that the swelling of P4VP film originates from the protonation of the pyridine units. (i) The pattern height v.s. bias voltages at various fixed contact forces. (j) The pattern height v.s. contact forces under different constant bias voltages $\ldots \ldots \ldots \ldots \ldots \ldots \ldots \ldots \ldots \ldots$

Fig. 6.3.3 Topographic images show the removal of the dot pattern at the center: (a) Initially, the dot height was $12 \mathrm{~nm}$; (b) After the first erasing performance, the height decreased to $7 \mathrm{~nm}$; (c) After the second erasing performance, the remaining height was only $3 \mathrm{~nm}$. Line profiles of (d), (e), and (f) correspond to the features selected in (a), (b), and (c), respectively $\ldots \ldots \ldots \ldots \ldots$ 


\section{CHAPTER 1. INTRODUCTION}

\subsection{Motivations of the Thesis}

The development of nanotechnologies and nanoscience implies a large effort to study and understand physical phenomena at the nanometer scale. Experiments using scanning probe methods based on shear-force control of the tip-sample distance provide great contributions to those studies. When a probe (in this case a tapered tip attached to a quartz tuning fork) is laterally oscillating and brought closer to a sample surface, its vibration amplitude is damped due to the shear-force effect. However, the origin of the shear-force damping mechanism is still not clear. Some authors suggest that one of the most important reasons for attenuation in the vibration amplitude is caused by the presence of a fluid-like contamination layer at the sample surface when working in ambient conditions.

Contamination layers are believed to be fluid-like films formed by adsorbed moisture and hydrocarbon molecules existent in the environment. The behavior of confined liquid films (i.e., the contamination layer constrained between probe and sample) is of critical importance in many fields. Examples include, among others, lubrication, adhesion, biological membranes, capillary forces in scanning probe microscopy and nanolithography. Herein, we have undertaken a further development of a novel tool for studying the nature of confined liquid layers including the mechanism of shear-force damping. By integrating acoustic transducers into the shear force microscope, the new shear-force/acoustic near-field microscope is capable of concurrently and independently monitoring the effects of liquid-mediated interactions acting on both microscope's probe 
and sample. It also provides an opportunity to have an insight into the properties of adsorbed fluid-like films on sample surfaces.

\subsection{Overview of Scanning Probe Microscopy}

Scanning probe microscopes are a family of instruments that allow scientists and engineers to measure properties of surfaces. The main characteristic of an SPM is its ability to accurately measure surface topography. This measurement is performed with a sharp probe, which is scanned over the sample surface while keeping a very small spacing to the surface. The family of SPMs encompasses scanning surface science tools for surface metrology. As opposed to optical microscopes and scanning electron microscopes (SEMs), they produce real space images with angstrom to nanometer resolution in all three dimensions: $\mathrm{x}, \mathrm{y}$ and $\mathrm{z}$.

\subsubsection{The Scanning Tunneling Current Microscope}

The Scanning Tunneling Microscope (STM) was the first SPM invented for imaging not only the structure of conducting layers but also electrical properties. The underlying physical principle of the STM is electron tunneling. ${ }^{[1]}$ It occurs between two conductors separated by a sufficiently thin insulating layer. For free electrons tunneling through a separating gap, the tunneling current I can be written as:

$$
I \propto V_{z} \exp \left(-A \Phi^{1 / 2} d\right)
$$

where $A=4 \pi(2 m)^{1 / 2} / h, I$ is the tunneling current, $V_{z}$ is the applied bias in volts, $\Phi$ is the average potential barrier height and $d$ is the spacing between the lowest atom on the tip and the highest atom on the sample. Thus, the operation of the STM is based on the strong exponential dependence of the tunneling current on the tip-sample separation, 
which is used to implement a feedback control that renders topographic images with nanometer precision. ${ }^{[2]}$

When performing an STM measurement, a very sharp metallic tip attached to the piezoelectric scanner is brought close enough to the substrate under investigation. The tip is scanned over the surface while the tunneling current (flowing between the probe and the sample) is simultaneously monitored. A bias voltage applied between the sample and the tip encourages the tunneling current to flow. The STM can be operated in either the constant current mode or the constant height mode. In the constant current mode, the STM tip closely tracks the sample surface, and a feedback circuit controls the height of the tip to maintain the tunneling current constant. The displacement of the tip given by the voltage applied to the piezoelectric scanner then yields a map of the surface topography. Alternatively, in the constant height mode, the feedback network responds only rapidly enough to keep the tip in an average (constant) distance from the sample surface. The tip can be scanned across the surface at nearly constant height and constant voltage, and the tunneling current is imaged. 


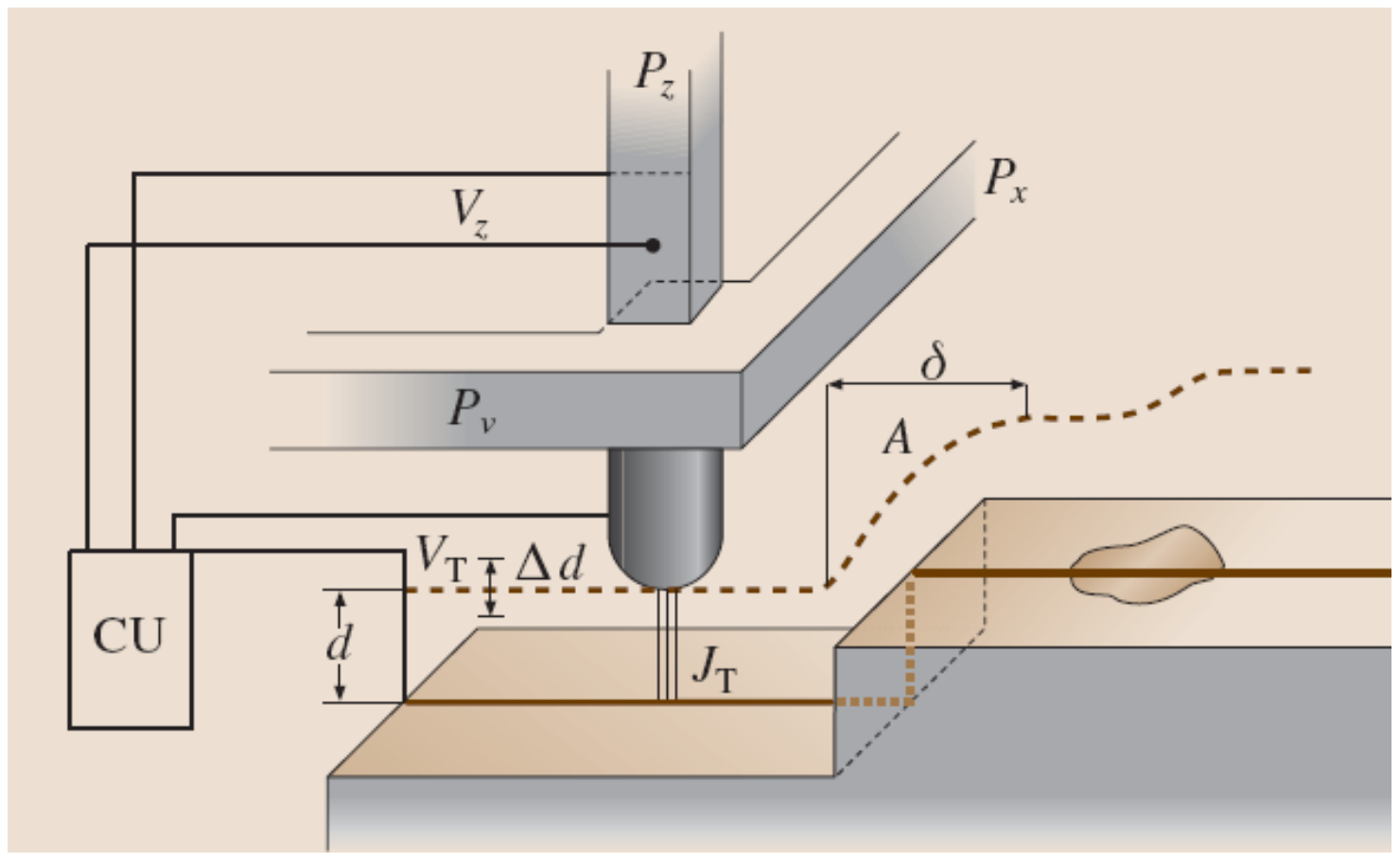

Fig. 1.2.1 The principle of the scanning tunneling microscope as sketched by Binning and Rohrer.

STM tips are typically fabricated out of metal wires of tungsten (W), platinumiridium (Pt-Ir), or gold $(\mathrm{Au})$, which are sharpened by grinding, cutting with a wire cutter or razor blade, controlled crashing, field emission/evaporator, ion milling, fracture, or electrochemical polishing/etching. The two most commonly used tips are made of either a Pt-Ir alloy or tungsten wire. The Pt-Ir tips are mechanically formed and are readily available; Iridium is used to provide stiffness. ${ }^{[3]}$ The $\mathrm{W}$ tips are etched form tungsten wire with an electrochemical process. ${ }^{[4]}$ These metal tips are very sharp and have high resolution, but are fragile and sometimes break when contacting a surface. Diamond tips made conductive by boron ion implantation have been developed to be used in STMs. ${ }^{[5]}$

Samples to be investigated with the STM must be electrically conductive to some degree to allow a few nanoamperes of current to flow from the bias voltage source to the area to be scanned. Nonconductive samples are generally coated with a thin layer of a 
conductive material to facilitate imaging. The bias voltage and the tunneling current depend on the sample; the different atomic species within a sample may produce different tunneling currents for a given bias voltage. Thus the height data may not be a direct representation of the topography of the surface of the substrate.

\subsubsection{The Atomic Force Microscope}

A few years after the invention of the STM, the Atomic Force Microscope (AFM) was developed based on the design of the STM to produce topographic images of surface atomic structure ${ }^{[6]}$ Instead of measuring tunneling current, the force present between a small tip and a sample surface of interest is detected in an AFM to sense the proximity of the tip to the sample. The AFM is a more versatile tool and has become a popular surface profiler for topographic and normal force measurements on the micro to nanoscale domains. Sample conductivity is no longer a requirement and so whole new classes of important materials, such as insulators and large band-gap semiconductor, are brought into the realm of atomic-scale scanning probe measurements.

In a typical AFM experiment, a sharp tip, attached to the free end of a flexible cantilever with a known spring constant, is brought into close proximity to a chosen substrate. The cantilever bends vertically in response to attractive and/or repulsive forces on the tip due to its interaction with the sample. ${ }^{[7]}$ The AFM can be operated either in a static or dynamic mode, ${ }^{[8]}$ and surface topographic information is provided by laterally scanning the sample under the tip while simultaneously measuring the separationdependent force or force gradient between the tip and the surface. In the static mode, also referred to as contact mode, an AFM tip is brought in contact with a given sample. Under the "contact" regime, a very weak repulsive force experienced by the tip causes a 
deflection of the cantilever from its equilibrium position. Therefore the force is detected by measuring the cantilever deflection. In the dynamic mode, also referred as to noncontact mode, the cantilever is sinusoidally vibrated and the oscillating tip is brought close to the sample surface. Interaction forces (i.e., van de Waals forces) existing in the tip-sample interface attract the tip to the sample, but the actual mechanical contact does not occur. So the force gradient is obtained by measuring the shift of resonant frequency and/or the attenuation of vibration amplitude of the oscillating cantilever.

For producing topographic images, the measurement of interaction forces is either recorded directly, or used as a control parameter for a feedback circuit that maintains the force or force gradient at a constant value. Currently, measuring the force is achieved by using the laser beam deflection technique. In an AFM system, a laser beam is reflected from the back of the cantilever into a position-sensitive quadrant photodetector (PSPD). When a sample moves under an AFM tip, features on the sample surface cause the cantilever to deflect. This tip deflection will change the direction of the reflected laser beam, changing the intensity difference between the top and bottom (left and right) sets of PSPD. This optical deflection method in fact measures the angles by which the cantilever is bent by interaction forces, which for small angles is linearly proportional to the tip deflection. ${ }^{[9]}$ This method is believed to be the most sensitive, reliable and easily implemented detection method and most commonly used in the commercial AFMs. 


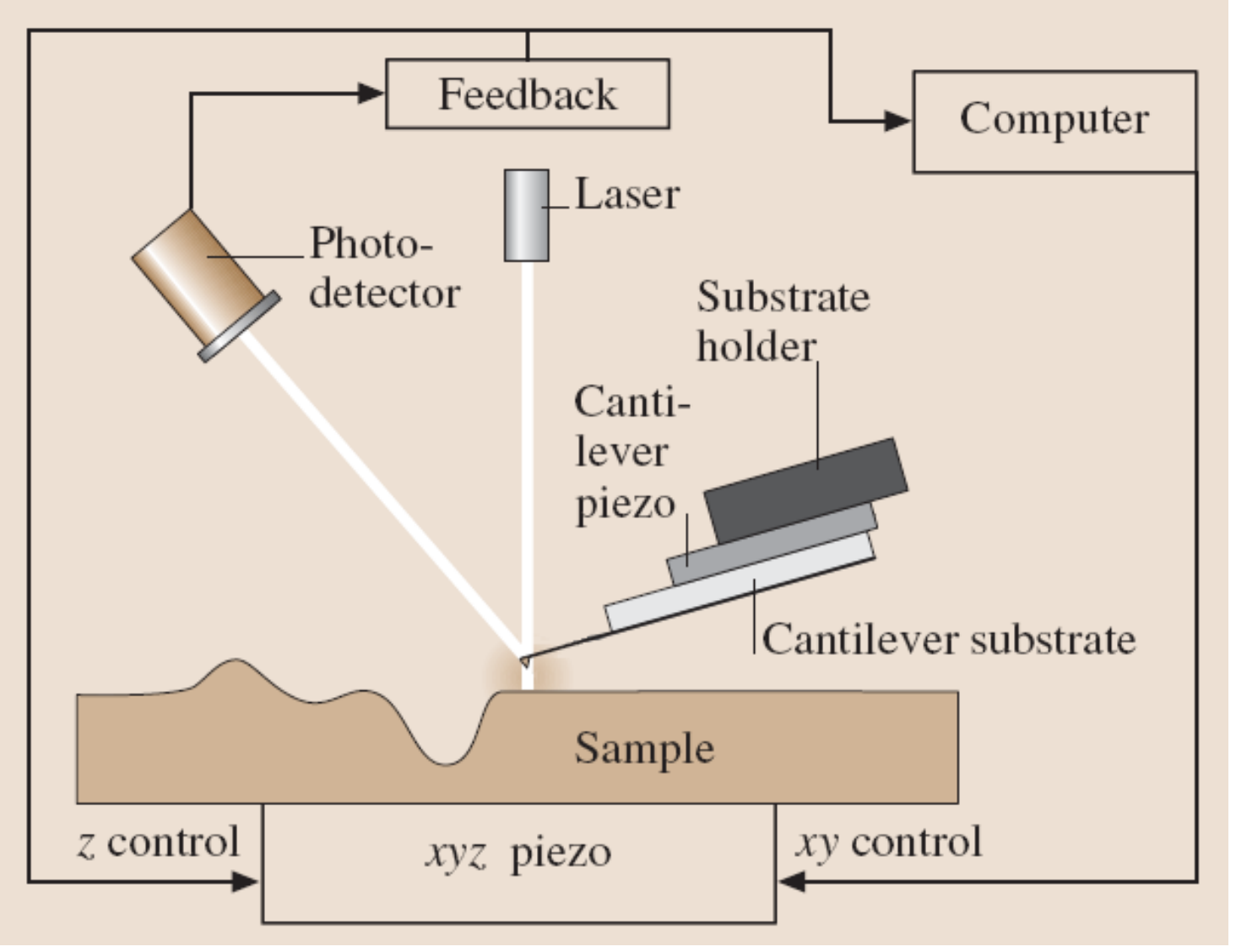

Fig. 1.2.2 The principle of the atomic force microscope as sketched by Bhushan.

AFM measurements can be performed in a variety of environments: ambient air, various controlled atmosphere, liquids, vacuum, at low temperatures and high temperatures. Imaging in liquid allows the study of live biological samples and it also eliminates water capillary forces present in ambient air at the tip-sample interface. ${ }^{[10]}$ Low temperature imaging is useful for the study of biological and organic materials and the study of low-temperature phenomena. ${ }^{[11]}$ Moreover, nanoscale science and technology are strongly driven by AFMs which allow investigation of surfaces down to the atomic scale. AFMs have been used to study the physics and chemistry of probesample interactions, as well as, quantitatively analyze tribological, electronic, magnetic, 
biological, and chemical surfaces. Besides being applied in basic research fields, AFMs are of practical importance and have been used for nanofabrication and nanomachining.

\subsubsection{The Shear-force/Acoustic Near-field Microscope}

The shear force microscope was first developed as a feedback mechanism in nearfield scanning optical microscopy (NSOM) ${ }^{[12]}$ Instead of oscillating the probe vertically, as the cantilever does in AFM tapping mode, the NSOM probe is excited to oscillate laterally with respect to the sample surface at its resonance frequency. When the tipsample distance is decreased below a few tens of nanometers, the so-called shear-force interactions between probe and sample are detected, leading to a decrease of vibration amplitude (sometimes along with a blue shift of resonance frequency). The shear-force interaction dependent on the tip-sample separation is so complex that the origin of the damping mechanism is still unclear. Currently, there is no satisfying theory to explain this interaction between probe and sample surface.

Quartz tuning fork (TF) nowadays has been introduced as the force sensor in shear force microscopes. ${ }^{[13]}$ The motion of TF modeled as the vibration of a damped single harmonic oscillator will be influenced by probe-sample interactions. By evaluating the change in electric signal of TF proportional to the applied force, interactions between probe and sample can be analyzed qualitatively and/or quantitatively. The implementation of TF greatly simplified force measurement schemes without losing too much sensitivity. Moreover, TF has advantages such as high quality factor, high stiffness, and no hazard to photosensitive materials. All these advantages over traditional silicon cantilevers broaden the application of TF-based shear force microscope in variety of areas. 
Conventionally, acoustics is used to characterize the internal microstructure of nontransparent materials. ${ }^{[14]}$ By incorporating an acoustic sensor into the shear force microscope, our group has developed a near-field scanning probe microscopy named Shear-force/Acoustic Near-field Microscopy (SANM). ${ }^{[15,16]}$ The SANM combines all of advantages of the shear force microscope and acoustics, providing the shear-force and acoustic interpretations of sample at the same time. Further improvements about the SANM will be discussed with more detail in next chapters.

\subsection{Outline of the Thesis}

This thesis is divided into eight chapters. Chapter 1 comprises the motivations of the thesis and the overview of scanning probe microscopy. Chapter 2 provides an introduction of theoretical studies with regard to surface phenomena. Chapter 3 focuses on the fabrication of metallic tips using gold wires. Key components of the shearforce/acoustic microscope system are introduced in this chapter as well. Chapter 4 presents the study of shear-force interactions between probe and sample. The experimental approach is based on a measurement of approach/retraction curves with a gold tip glued to a tuning fork and a silicon substrate. Tips with different shapes are used to study the influence of tip geometry. The influence of environmental humidity is studied as well. Furthermore, chemical treatments of the tip and sample allow studying the differences of the tip-sample interaction when water affinity of tip and sample surfaces is changed. Chapter 5 concentrates on the study of contact current used to indicate probe-sample contact, as well as the correlations among the contact current, shear-force and acoustic signals. Chapter 6 demonstrates the fabrication of patterns on 
poly(4-vinylpyridine) (P4VP) thin films based on dip-pen nanolithography (DPN) technique by exploiting the swelling of the polymer. Final conclusions and future recommendation are presented in chapter 7 and 8 , respectively. 


\section{CHAPTER 2. THEORETICAL STUDIES UNDERLYING THE EXPERIMENTS}

\subsection{Adsorption}

The concept of adsorption is of great importance to both theoretical and practical understanding of interfacial phenomena. Adsorption can be most simply defined as the taking up of one substance at the surface of another. There are several underlying principles of adsorption that are fundamental for understanding the all adsorption phenomena, especially the Gibbs adsorption equation, the Young-Laplace equation, and the Kelvin equation, which will be introduced in turn.

\subsubsection{The Gibbs Adsorption Equation}

The Gibbs adsorption equation describes the changes in the surface tension with variances in the concentration of a species in contact with a surface. ${ }^{[17]}$ In order to define the state of a bulk phase $\alpha$, it is necessary to specify the values of several variables associated with the state. Those variables include the temperature $T^{\alpha}$, its volume $V^{\alpha}$, and the system pressure $P^{\alpha}$. In terms of the Helmholtz free energy $A$, the system can be specified by: ${ }^{[17]}$

$$
A^{\alpha}=-S^{\alpha} T^{\alpha}-P^{\alpha} V^{\alpha}+\sum \mu_{i}^{\alpha} n_{i}^{\alpha}
$$

or in differential terms (with constant $P$ ):

$$
d A^{\alpha}=-S^{\alpha} d T^{\alpha}-P^{\alpha} d V^{\alpha}+\sum \mu_{i}^{\alpha} d n_{i}^{\alpha}
$$

where $S^{\alpha}$ is the entropy, $n_{i}^{\alpha}$ and $\mu_{i}^{\alpha}$ are the amounts and chemical potentials of species $i$.

For a system of two phases, a similar equation can be written for the second phase $\beta$. At equilibrium, the two phases will have the same temperature $T$, the same pressure $P$, and the same chemical potential $\mu$, for all components. Moreover, the $\alpha-\beta$ interface 
present at the system of two phases in contact is considered to be a third phase $\sigma$. Then the total energy will be written as:

$$
A^{T}=A^{\alpha}+A^{\beta}+A^{\sigma}
$$

where $A^{\sigma}$ is the interfacial free energy. Analogous to eq. (2.1.1), the derivative of the interfacial free energy is given by:

$$
d A^{\sigma}=-S^{\sigma} d T+\gamma d A_{S}+\sum \mu_{i} d n_{i}^{\sigma}
$$

where $\gamma$ is the surface or interfacial tension between $\alpha$ and $\beta, A_{S}$ is the interfacial area, and $\mu_{i}$ has the same value as that in the bulk phase.

In the area of bulk thermodynamics, integration of eq. (2.1.1) while holding $T, P$, and $\mu_{i}$ constant yields:

$$
A^{\alpha}=-P V^{\alpha}+\sum \mu_{i}^{\alpha} n_{i}^{\alpha}
$$

At equilibrium, taking the differential of $A^{\alpha}$ and substituting eq. (2.1.1) then yields:

$$
-S^{\alpha} d T+V^{\alpha} d P+\sum n_{i}^{\alpha} d \mu_{i}^{\alpha}=0
$$

Therefore, an identical process for the interfacial "phase" yields

$$
-S^{\sigma} d T-A_{S} d \gamma+\sum n_{i}^{\sigma} d \mu_{i}=0
$$

At constant temperature, eq. (2.1.2) reduces to:

$$
-d \gamma=\sum n_{i}^{\sigma} \partial \mu_{i} / A_{S}
$$

where $\Gamma_{i}=n_{i}^{\sigma} / A_{S}$, defined as a concentration termed the surface excess concentration of $i$. Therefore, the Gibbs Adsorption Equation in term of surface excess is:

$$
-d \gamma=\sum \Gamma_{i} d \mu_{i}
$$


For dilute solutions containing one non-dissociating surfactant, a more general form of the Gibbs adsorption equation is:

$$
d \gamma=-R T \Gamma_{2}^{(1)} d \ln C_{2}
$$

in which 2 designates the solute dissolved in bulk phase 1, and $C_{2}$ denotes the molar concentration of surfactant in bulk. The practical applicability of this equation is that the relative adsorption of a material at an interface can be determined from measurement of the interfacial tension as a function of solute concentration:

$$
\Gamma_{2}^{(1)}=-\frac{1}{R T}\left(\frac{d \gamma}{d \ln C_{2}}\right)
$$

\subsubsection{The Young-Laplace Equation}

The Young-Laplace equation describes the pressure difference across a curved interface in terms of surface tension and curvature. ${ }^{[18]}$ Since many practical situations involve surfaces and interfaces with high degrees of curvature, it is important to understand the effect of curvature on interfacial properties. If a liquid-fluid interface is curved, there will be a difference in hydrostatic pressure across the interface due to the tension existing at the interface. An expression has been derived for the pressure difference across a curved interface in terms of surface tension and curvature. The equation, referred as the Young-Laplace equation, is: ${ }^{[18]}$

$$
P_{1}-P_{2}=\Delta P=\gamma\left(\frac{1}{r_{1}}+\frac{1}{r_{2}}\right)
$$

in which $P_{1}$ and $P_{2}$ are the pressure in the two phases forming the interface, $r_{1}$ and $r_{2}$ are the principal radii of curvature of the interface, and $\gamma$ is the surface or interfacial tension. In particular, for a spherical surface $r_{1}=r_{2}=r$, the equation simplifies to: 


$$
\Delta P=2 \gamma / r
$$

The sign of the pressure term in eq. (2.1.3) will depend on the assignment of $P_{1}$ and $P_{2}$; however, convention is to assign $P_{1}$ to the liquid phase of interest and $P_{2}$ to the adjoining fluid phase. In a plane surface where $r_{1}=r_{2}=\infty, \Delta P=0$. Where the surface is concave inward (toward the liquid), $\Delta P>0$; where it is convex inward, $\Delta P<0$. Where a pressure differential exists, the liquid will flow from high to low pressure until the differential is decreased and finally disappear. In circumstances where the interfacial tension is uniform from point to point, the pressure will depend only on the curvature of the interface.

\subsubsection{The Kelvin Equation}

The Kelvin equation describes the variation of vapor pressure as a function of the curvature of the liquid-vapor interface. ${ }^{[19]}$ For a very small drop of liquid in which there is a large surface to volume ratio, the vapor pressure is higher than that over a plane surface of equal area. The movement of liquid from a flat interface into a volume with a curved interface requires the input of energy into the system since the surface free energy of the curved volume increases. If the radius of a drop is increased by a factor $d r$, the surface area increases from $4 \pi r^{2}$ to $4 \pi(r+d r)^{2}$, or by a factor of $8 \pi r d r$. Therefore, the free energy increase is given by: ${ }^{[19]}$

$$
\Delta G=\gamma \times 8 \pi r d r
$$

where $\gamma$ is the surface tension of the adsorbed film. If during the process $d n$ moles of liquid are transferred from the plane surface with a equilibrium vapor pressure $P_{0}$ to the drop with vapor pressure $P$, the free energy increase is also given by: 


$$
\Delta G=R T \ln \left(P / P_{0}\right) d n
$$

Equating the two relationships yield the expression known as the Kelvin equation:

$$
R T \ln \left(P / P_{0}\right)=2 \gamma V_{m} / r
$$

where $V_{m}$ the molar volume of the liquid, $R$ is the ideal gas constant, $T$ is the absolute temperature.

\subsubsection{Physical Adsorption and Chemisorption}

Adsorption can occur at any type of interface; however, interfaces containing only liquids and vapors exhibit slightly simpler adsorption characteristics than those containing solid surfaces. The reason is that in a liquid surface, the complications resulted from surface heterogeneities and specific structures can be ignored. A freshly prepared solid surface will often be of quite high surface energy so that there will exist a strong driving force for the reduction of the excess surface energy. The forces involved in adsorption process include the nonspecific van de Waals forces, ionic or electrostatic forces, and specific forces involved in the formation of chemical bonds. Adsorption processes that involve only nonspecific interactions are referred to as physical adsorption while those in which stronger specific interactions take place are termed chemisorption.

In the absence of complicating factors such as capillary condensation, the process of physical adsorption has no activation energy; it is diffusion controlled and occurs as rapidly as vapor molecules can arrive at the solid surface. The process will have reversible character and equilibrium will be attained rapidly. Furthermore, physical adsorption will be a multilayer process; that is, the amount of vapor which can be adsorbed onto a surface will not be limited by the available solid surface area, but molecules can stack up to several molecular thickness in a pseudo-liquid assembly. 
Unlike physical adsorption, chemisorption involves very specific interactions between the solid surface and the adsorbing molecules, generally forming a monomolecular adsorbed layer. Additionally, chemisorption process will have some activation energy and may thereby be much slower than physical adsorption, and it may not exhibit reversibility.

\subsection{Adhesion}

Adhesion is an extremely important concept in both theoretical and practical terms. A practical definition is a state in which two bodies are held together by intimate interfacial contact in such a way the mechanical force or work can be applied across the interface without causing these two bodies to separate. Thermodynamic adhesion is the term that applies to the "ideal" adhesion defined in terms of reversible work needed to separate two surfaces by overcoming the molecular interactions across the interface. Mechanical adhesion is the term that may be applied to the situation in which actual mechanical interlocking of microscopic asperities at the interface occurs over a significant portion of the contact area. Chemical adhesion refers to adhesion involving the formation of formal chemical bonds (covalent, electrostatic, or metallic) across an interface. Before entering into the topic of adhesion, it is useful to introduce several terms, such as surface tension, interfacial tension, work of adhesion and work of cohesion.

\subsubsection{Work of Adhesion and Work of Cohesion}

For two different media 1 and $2(1 \neq 2)$, the work of adhesion $W_{a(12)}$ is defined as the free energy change, or the reversible work done, to separate unit area of interface between these two materials from contact to infinity in vacuum (Fig. 2.2.1a). The work of adhesion is given by: 


$$
W_{a(12)}=\gamma_{1}+\gamma_{2}-\gamma_{12}
$$

where the subscripts refer to the two media being separated, and the $\gamma$ 's are the respective surface or interfacial tensions. For two identical media $(1=2)$, related to $W_{a(12)}$ is the work of cohesion $W_{c(11)}$, defined as the reversible work required to separate two surfaces of unit area of the material (Fig. 2.2.1b). It represents a minimum amount of work for the creation of two unit areas of fresh surface. The work of cohesion is:

$$
W_{c(11)}=2 \gamma_{1}
$$

Since all media attract each other in vacuum $W_{a(12)}$ and $W_{c(11)}$ are always positive.

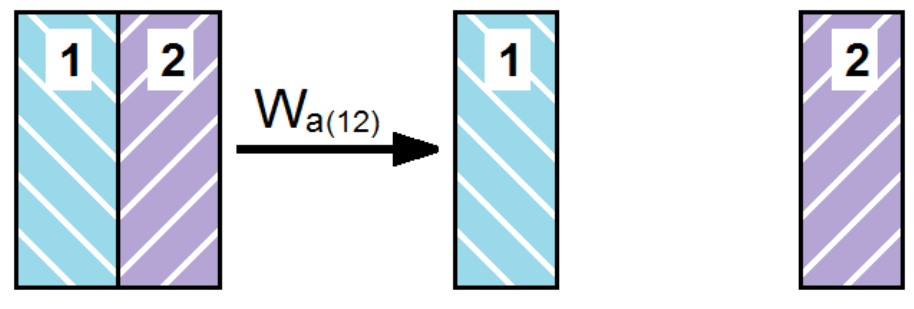

(a) Work of Adhesion, $\mathrm{W}_{\mathrm{a}(12)}$

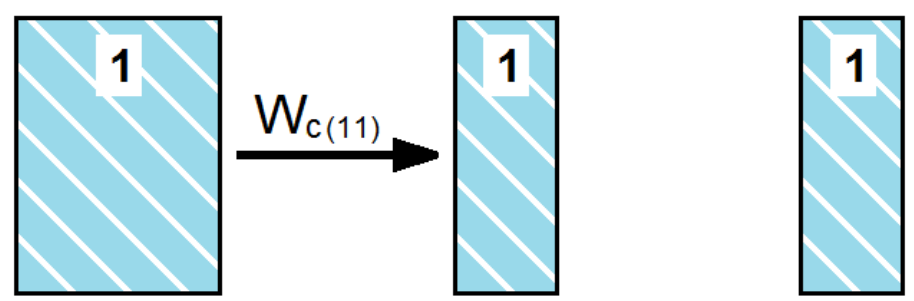

(b) Work of Cohesion, $\mathrm{W}_{\mathrm{c}(11)}$

Fig. 2.2.1 Definitions of the works of adhesion and cohesion.

\subsubsection{Surface Tension and Interfacial Tension}

Surface tension $\gamma$ is the free energy change when the surface area of a medium is increased by unit area. Since the process of creating unit area of surface is equivalent to separating two half-unit areas from contact, the surface tension can be written as: 


$$
\gamma=\frac{1}{2} W_{c}
$$

For solids $\gamma$ is commonly denoted by $\gamma_{S}$ and is given in units of energy per unit area: $\mathrm{mJm}^{-2}$. For liquids $\gamma$ is commonly denoted by $\gamma_{L}$ and is given in units of tension per unit length: $\mathrm{mNm}^{-1}$, which is numerically and dimensionally the same as the surface free energy.

When two media 1 and 2 are in contact, the free energy change in expanding their interfacial area by unit area is known as their interfacial tension $\gamma_{12}$. The energetics associated with this expansion process may consist of two hypothetical steps: first unit areas of media 1 and 2 are created, and are then brought into contact. Therefore the total free energy change is:

$$
\gamma_{12}=\frac{1}{2} W_{c(11)}+\frac{1}{2} W_{c(22)}-W_{a(12)}=\gamma_{1}+\gamma_{2}-W_{a(12)}
$$

which is referred to as the Dupre equation. This energy is formally the same as that expended on separating two media 1 in medium $2\left(W_{121}\right)$ or, of separating two media 2 in medium $1\left(W_{212}\right)$. So $\gamma_{12}$ can be also written as:

$$
\gamma_{12}=\frac{1}{2} W_{121}=\frac{1}{2} W_{212}
$$

In addition, the energy change on separating two media 1 and 2 in medium 2 is given by:

$$
W_{132}=W_{a(12)}+W_{c(33)}-W_{a(13)}-W_{a(23)}=\gamma_{13}+\gamma_{23}-\gamma_{12}
$$

\subsubsection{Ideal Adhesion}

In theory, the concept of thermodynamic adhesion applies equally well to liquid and solid phases. However, it does not take into consideration conditions before or after the formation of the interface, the presence of random flaws or defects in the system, or the 
bulk physical properties of the components. The complete expression for the work of adhesion between two phases with each phase completely saturated by the other is:

$$
W_{A(B) B(A)}=\gamma_{A(B)}+\gamma_{B(A)}-\gamma_{A B}
$$

The maximum force required to separate unit area of interface, or the ideal adhesive strength of the interface $F_{a}$ can be estimated by: ${ }^{[20]}$

$$
F_{a}=1.03 W_{A(B) B(A)} / r_{0}
$$

where $r_{0}$ is the equilibrium distance of separation on the order of a few molecular diameters. Similar calculations can be made for cohesive strength as well.

\subsubsection{Adhesion Force between Solid Particles}

When real particles come into contact, they deform elastically under the influence of any externally applied load as well as the attractive interface forces that pull the two surfaces together. This results in a finite contact area even under zero external loads. Hertz was the first to describe the mechanical behavior of two elastic spheres (or a sphere and a plane surface) pressed together. ${ }^{[21]}$ In the theory any surface interactions such as near contact Van der Waals interactions, or contact adhesive interactions are neglected.

An improvement over Hertzian theory was achieved by Jonhnson et al. with the

JKR (Johnson, Kendall and Roberts) theory. ${ }^{[22]}$ In JKR theory the contact is considered to be adhesive. Hence the theory correlates the contact area to the elastic materials properties plus the interfacial interaction strength. Due to the adhesive contact, contacts can be formed during the unloading cycle also in the negative loading (pulling) regime. However, the JKR theory is still restricted to elastic sphere-sphere contacts such as the Hertzian theory. 
Another theory by Derjaguin, Muller and Toporov (DMT theory) considers Van der Waals interactions outside the elastic contact regime, which give rise to an additional load. The theory simplifies to Bradley's van der Waals model if the two surfaces are separated and significantly apart. In Bradley's model any elastic deformations due to the effect of attractive interaction forces are neglected.

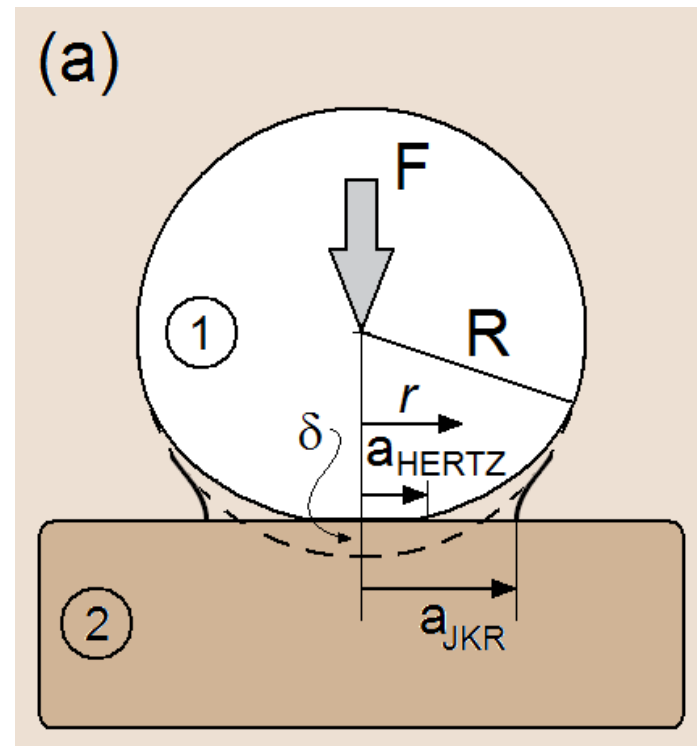

\section{Elastic sphere: Equilibrium}

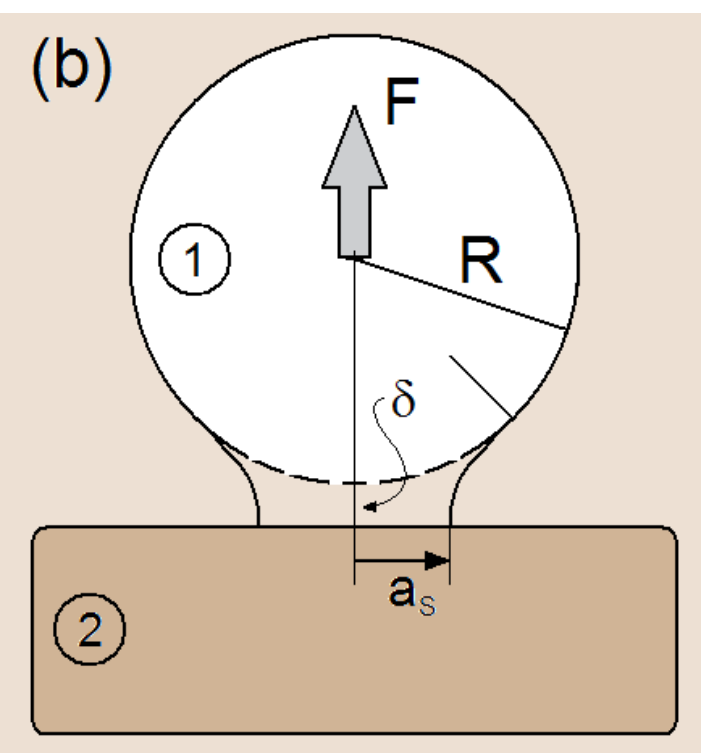

Elastic sphere: Pull-off

Fig. 2.2.2 (a) Elastic sphere on rigid surface in the absence (Hertz) and presence (JKR) of adhesion. (b) Elastic adhering sphere about to separate spontaneously from adhesive contact.

In the JKR theory, we will consider the case in that two elastic spheres of radii $R_{1}$ and $R_{2}$, bulk elastic modulus $K$, and the work of adhesion $W_{12}$, are pressed together under an external load of normal force $F$. Therefore, at mechanical equilibrium the radius of their contact area $a$ is given by: ${ }^{[23]}$

$$
a^{3}=\frac{R}{K}\left[F+3 \pi R W_{12}+\sqrt{6 \pi R W_{12} F+\left(3 \pi R W_{12}\right)^{2}}\right]
$$


where $R=R_{1} R_{2} /\left(R_{1}+R_{2}\right)$. For a sphere of radius $R$ and a plane surface of the same material (Fig. 2.2.2a), we have $R_{2}=\infty, R=R_{1}$ and $W_{12}=2 \gamma_{S V}$ (sphere on flat surface in vapor), so that under zero load ( $F=0$ ) the contact radius is given by:

$$
a_{0}=\left(6 \pi R^{2} W_{12} / K\right)^{1 / 3}=\left(12 \pi R^{2} \gamma_{S V} / K\right)^{1 / 3}
$$

Furthermore, under small negative loads $(F<0)$ the solids still adhere until at some critical negative force the surfaces suddenly jump apart (Fig. 2.2.2b). This adhesion or "pull-off" force is given by:

$$
F_{s}=-3 \pi R \gamma_{S V}
$$

and separation takes place suddenly once the contact radius has fallen to:

$$
a_{s}=a_{0} / 4^{1 / 3}=0.63 a_{0}
$$

The central displacement $\delta$ is given by:

$$
\delta=\frac{a^{2}}{R}\left[1-\frac{2}{3}\left(\frac{a_{0}}{a}\right)^{3 / 2}\right]
$$

The pressure or stress distribution within the contact circle is given by:

$$
p(x)=\frac{3 K a}{2 \pi R}\left(1-x^{2}\right)^{1 / 2}-\left(\frac{3 K W_{12}}{2 \pi a}\right)\left(1-x^{2}\right)^{-1 / 2}
$$

where $x=r / a$.

In the absence of adhesive interactions, the equations of the JKR theory reduce to the expressions in the Hertz model:

Adhesion force: $F_{S}=0$

Contact radius: $a^{3}=R F / K$

Displacement: $\delta=a^{2} / R=F / K a$ 
Pressure: $p(x)=\frac{3 K a}{2 \pi R}\left(1-x^{2}\right)^{1 / 2}=\frac{3 F}{2 \pi a^{2}}\left(1-x^{2}\right)^{1 / 2}$

One difficulty with the JKR theory is that it predicts an infinite stress at the edge of the contact circle where the surface is expected to bend infinitely sharply through $90^{\circ}$. However, this infinity disappears as long as the attractive force law between the surfaces is allowed to have a finite range. One simple modified conclusion is that in the limit of small deformations the adhesion force changes from the "JKR limit" of $F_{s}=-3 \pi R \gamma_{S V}$ to the so-called "DMT limit" of $F_{s}=-4 \pi R \gamma_{S V}$. Another more practical limitation of the JKR and Hertzian theories is that they assume perfectly smooth surfaces. Real surfaces are rough, but there is as yet no satisfactory theory for such real world situations.

\subsection{Effect of Capillary Condensation}

When considering the adhesion of two solid surfaces or particles in air or in liquid, the water capillary force is inevitably present and may play a significant role. Capillarity is a general phenomenon that arises as a result of differences in pressure across a system containing at least one liquid phase and another liquid, vapor and/or solid phase. For a sphere-on-plane geometry, the capillary forces may arise from the Laplace pressure of curved menisci formed by condensation of a liquid between and around these two adhering surfaces (Fig. 2.3a). 

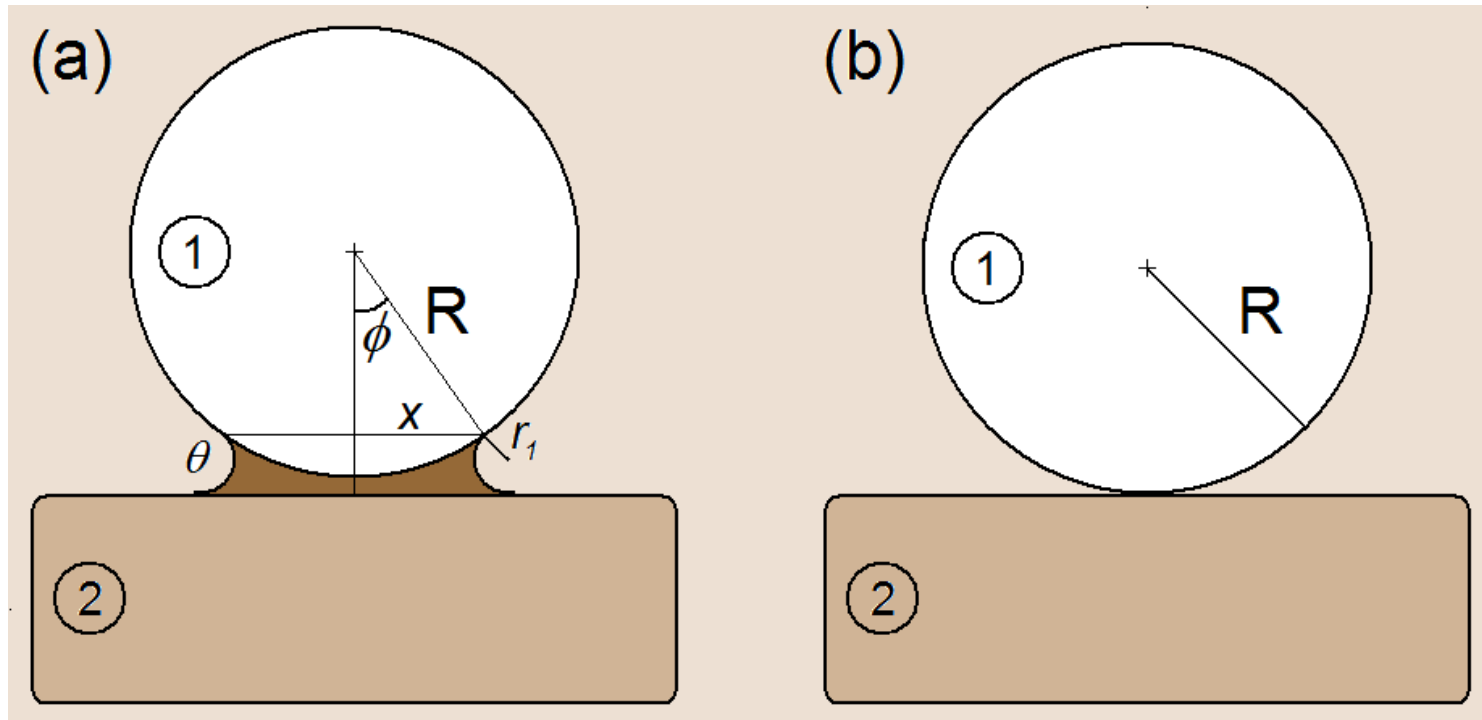

Fig. 2.3 A non-deforming sphere on a rigid flat surface: (a) In a vapor the capillary force arises from the Laplace pressure. (b) In the absence of condensing vapor, the adhesion force is due to the direct contact of the surfaces.

A simple derivation of the capillary force is given by considering the Laplace pressure in the liquid as: ${ }^{[24]}$

$$
P=\gamma_{L}\left(\frac{1}{r_{1}}+\frac{1}{r_{2}}\right) \approx \frac{\gamma_{L}}{r_{1}} \quad\left(\text { since } r_{2} ? r_{1}\right)
$$

The Laplace pressure acts on an area $\pi x^{2} \approx 2 \pi R d$ between the two surfaces, thereby combining them together with a force:

$$
F \approx 2 \pi R d \frac{\gamma_{L}}{r_{1}}
$$

For small $\phi, d \approx 2 r_{1} \cos \theta$, and the Laplace pressure contribution to the adhesion force is given by:

$$
F \approx 4 \pi R \gamma_{L} \cos \theta
$$

where $\gamma_{L}$ is the surface tension of the liquid and $\theta$ is the contact angle, which is assumed to be the same on the two solid surfaces. The additional force arising from the resolved 
surface tension around the circumference is always small compared to the Laplace pressure contribution except for $\theta \approx 90^{\circ}$. More rigorous expressions, valid for large $\phi$ and different contact angles on each surface, are given by Orr et al..

For the direct solid-solid contact adhesion force in an atmosphere containing a condensable vapor, the expression is written as:

$$
F=4 \pi R\left(\gamma_{L} \cos \theta+\gamma_{S L}\right)
$$

where the first term is due to the Laplace pressure of the meniscus and the second is due to the direct adhesion of the two contacting solids within the liquid. In the absence of any condensing vapor (Fig. 2.3b), the above reduces to:

$$
F=4 \pi R \gamma_{S} \text { (sphere on flat surface in vacuum) }
$$

The capillary condensation of liquids, especially water, from vapor can have additional effects on the physical state of the contact region. If the surfaces contain ions, these will diffuse within the liquid bridge, thereby changing the chemical composition of the contact zone, as well as influencing the adhesion.

\subsection{Contact Angle}

When a droplet of liquid is placed on a solid surface, the liquid will either spread across the surface to form a uniform film or is will spread to limited extent but remain as a discrete drop on the surface. The condition of the dropped liquid on the surface is taken as an indication of the wettability of the surface by the liquid or the wetting ability of the liquid on the surface. The quantitative measure of the wetting process is taken to be the contact angle $\theta$, which can be considered as a characteristic of the surface. 


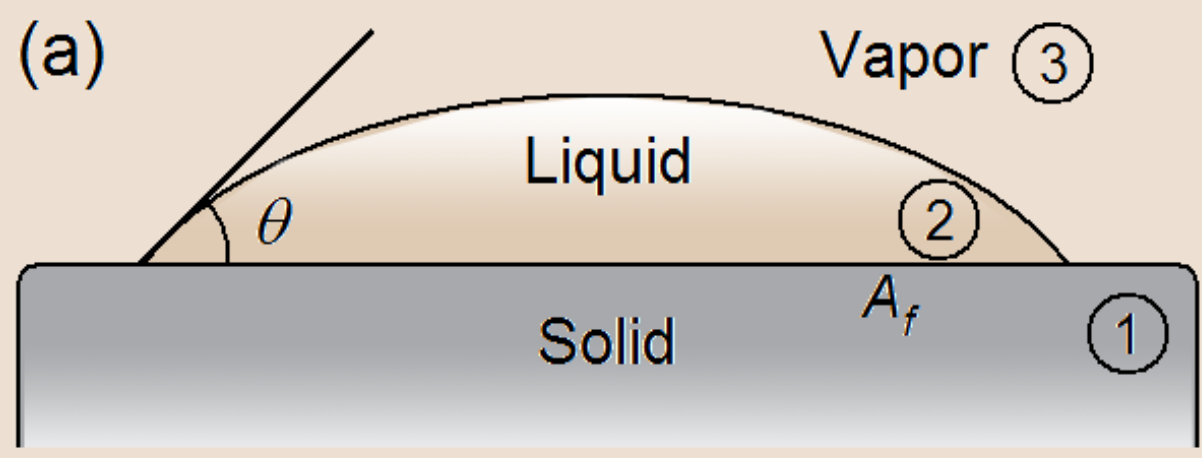

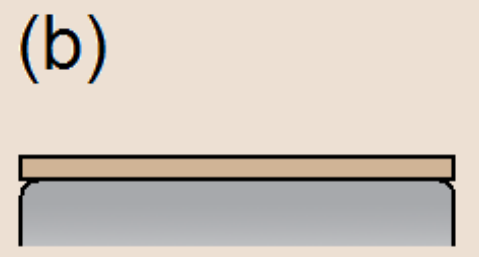

complete wetting (c)

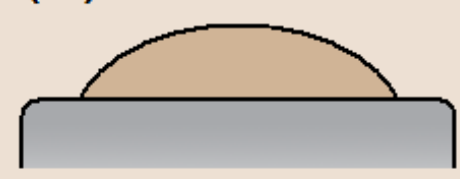

partial wetting (d)

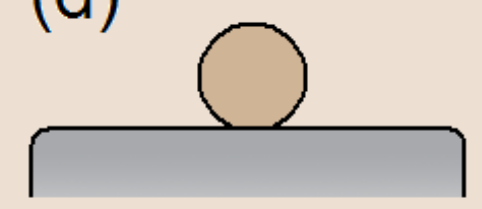

complete nonwetting

Fig. 2.4 (a) Schematic of a droplet on a solid surface and the definition of the contact angle. (b-d) The various degrees of wetting: complete wetting, partial wetting, and complete nonwetting.

Surface and interfacial energies determine how liquid droplets deform when they adhere to a surface. As shown in Fig. 2.4a, a spherical droplet 2 in medium 3 settles on the rigid flat surface 1 . The final total surface energy of the system is given by: ${ }^{[25]}$

$$
W_{t o t}=\gamma_{23}\left(A_{c}+A_{f}\right)-W_{132} A_{f}
$$

where $A_{c}$ and $A_{f}$ are the curved and flat areas of the droplet. At equilibrium:

$$
0=\gamma_{23}\left(d A_{c}+d A_{f}\right)-W_{132} d A_{f}
$$

For a droplet of constant volume, $d A_{c} / d A_{f}=\cos \theta$, thereby the equilibrium condition $\left(\theta=\theta_{0}\right)$ is:

$$
\gamma_{23}\left(1+\cos \theta_{0}\right)=W_{132}=\gamma_{13}+\gamma_{23}-\gamma_{12}
$$

or

$$
\gamma_{12}+\gamma_{23} \cos \theta_{0}=\gamma_{13}
$$


The above equation is more generally known as the famous Young's equation derived for liquid droplets on surfaces exposed to vapor:

$$
\gamma_{S V}=\gamma_{S L}+\gamma_{L V} \cos \theta
$$

It is often found that contact angles often exhibit hysteresis. When a liquid spreads and then retracts from a surface the advancing contact angle $\theta_{A}$ is generally larger than the receding angle $\theta_{R}$. Contact angle hysteresis $\left(\theta_{A}>\theta_{R}\right)$ actually implies adhesion hysteresis, $W_{R}>W_{A}$, where and are the adhesion or surface energies for receding (separating) and advancing (approaching) two surfaces, respectively. ${ }^{[26]}$ Due to the existence of contact angle hysteresis, it is always important to know as much as possible about the cleanliness, topography, homogeneity, etc, of a solid surface, as well as the purity and composition of the liquid employed, when attempting to interpret contact angle data.

\subsection{Hydrophobic/Hydrophilic Interactions}

All molecules including polar and non-polar molecules attract each other at distance of the order of a few Angstrom $(\AA)$ by weak attractive force. As is known, water molecules can form "strong" intermolecular interactions, so-called "hydrogen bond", whose strength is always between van der Waals force and covalent bonds. The polarity and H-bonding strength of water make it a unique solvent and highly active molecule, so when any molecules are dissolved in water, polarity and H-bond are playing important roles in the interaction formation between solutes and water molecules.

"Hydrophobic" means "water-hating (or fearing)", and it always refers to the category of substance which is immiscible with water. Similarly, if the surfaces cannot be 
wetted by water, forming a large contact angle (where liquid/vapor interface meets the solid surface), they are also hydrophobic. Hydrophobic interaction is defined as the unusually strong attraction between two hydrophobic molecules and surfaces when they are immersed in water, and experimental results show that it exceeds the expected van der Waals force at large separations by more than one order of magnitude. ${ }^{[27]}$ This strong interaction summarizes all of forces which reduce the solubility of non-polar molecules in water, but the exact molecular mechanism is still unknown because the hydrophobic interaction between two molecules is complex, it involves many other molecules and is of much longer range than a typical covalent bond. Now it is assumed that the interaction arises from the configurational rearrangement of water molecules as two hydrophobic species come together. The restructuring of water molecules is entropically unfavorable, in other words, rather than forming new structure with non-polar solutes, water prefer maintaining the existing interactions with same water molecules. Hydrophobic interaction plays a major role in many surface phenomena, in molecular self-assembly, in biological membrane structure and in the formation of the compact structure of proteins.

In contrast, "hydrophilic" means "water-loving", and hydrophilic groups prefer to be in contact with water rather than with each other. Hydrophilic interaction is the summation of all intermolecular interactions (including electrostatic) that cause the process of hydration (or salvation, the clustering process of water molecules around a solute particle). It is clear that polar molecules are easy to dissolve in water, because it requires low energy to break the H-bonds already established among water molecules and induce new H-bonds formation which is participated by polar solute molecules. But some uncharged and even non-polar molecules can be hydrophilic if they have the right 
geometry and if they contain electronegative atoms capable of associating with the $\mathrm{H}$ bond network in water. 


\section{CHAPTER 3. FABRICATION OF METALLIC PROBES AND EXPERIMENTAL INSTRUMENTATION}

\subsection{Fabrication of Tapered Gold Tips}

In apertureless scanning probe microscopy (SPM) sharp metallic tips are used to probe the sample properties at the nanometer scale. Several methods have been developed to fabricate tips. Among these methods, mechanical cutting is apparently the easiest way, but the reproducibility of this method is poor. Ideal tips with a single atom at the tip can be prepared in field ion microscope in UHV, but this is an expensive and complicated method not affordable for most researchers. The most practical way of preparing metallic tips is using electrochemical etching. For example, tungsten tips, which are commonly used as metallic tips in SPM, can be easily fabricated using

electrochemical etching. ${ }^{[4]}$ However, a drawback of tungsten tips is that tungsten will slowly oxidize in air. Instead of tungsten, gold is preferred for use in air. It is nearly impossible to form oxide layers outside gold tips because gold is a noble metal. Herein, we demonstrate an easy and low cost procedure for the fabrication of tapered gold tips based on electrochemical etching in hydrochloric acid.

\subsubsection{Experimental Section}

Gold tips are fabricated by electrochemical etching gold wires (Au, purity $99.95 \%$, ESPI) with diameters of 0.004 inches $(100 \mu \mathrm{m})$ and 0.003 inches $(76 \mu \mathrm{m})$. The experimental setup for etching gold wires is based on the one used to etch tungsten wires, as shown in Fig. 3.1.1. The etching voltage used to make the gold tip is supplied by a home-made DC power supply. A wire mount fixed to z-direction translator allows the immersion depth of gold wires to be precisely adjusted. An electronic microcontroller is 
inserted in the circuit to monitor the current after the etching process starts. A homemade controlling circuit acts as a quick switch to cut off the current once the etching process is completed. A small ring of about $3 \mathrm{~mm}$ diameter made from 0.008 inches (200 $\mu \mathrm{m}$ ) gold wire (Au, purity $99.95 \%$, ESPI) is used as the cathode electrode. The gold wire that will be etched threads through the ring, acting as the anode electrode.

The etching of gold wires takes advantage of the dissolution of gold in a solution containing chloride ions $\left(\mathrm{Cl}^{-}\right)$. The electrolyte solution is prepared by mixing hydrochloric $(\mathrm{HCl})$ acid and ethanol. The advantage of using $\mathrm{HCl}$ acid as the electrolyte solution is that it is required a relatively low voltage (i.e., $5 \mathrm{~V}$ ) for the etching process to take place. A droplet of the electrolyte solution is suspended inside the gold ring by surface tension. Once the etching process is completely, the formed tip is quickly removed from the electrolyte solution and cleaned in isopropyl alcohol (IPA) with ultrasonic for 1 minute.

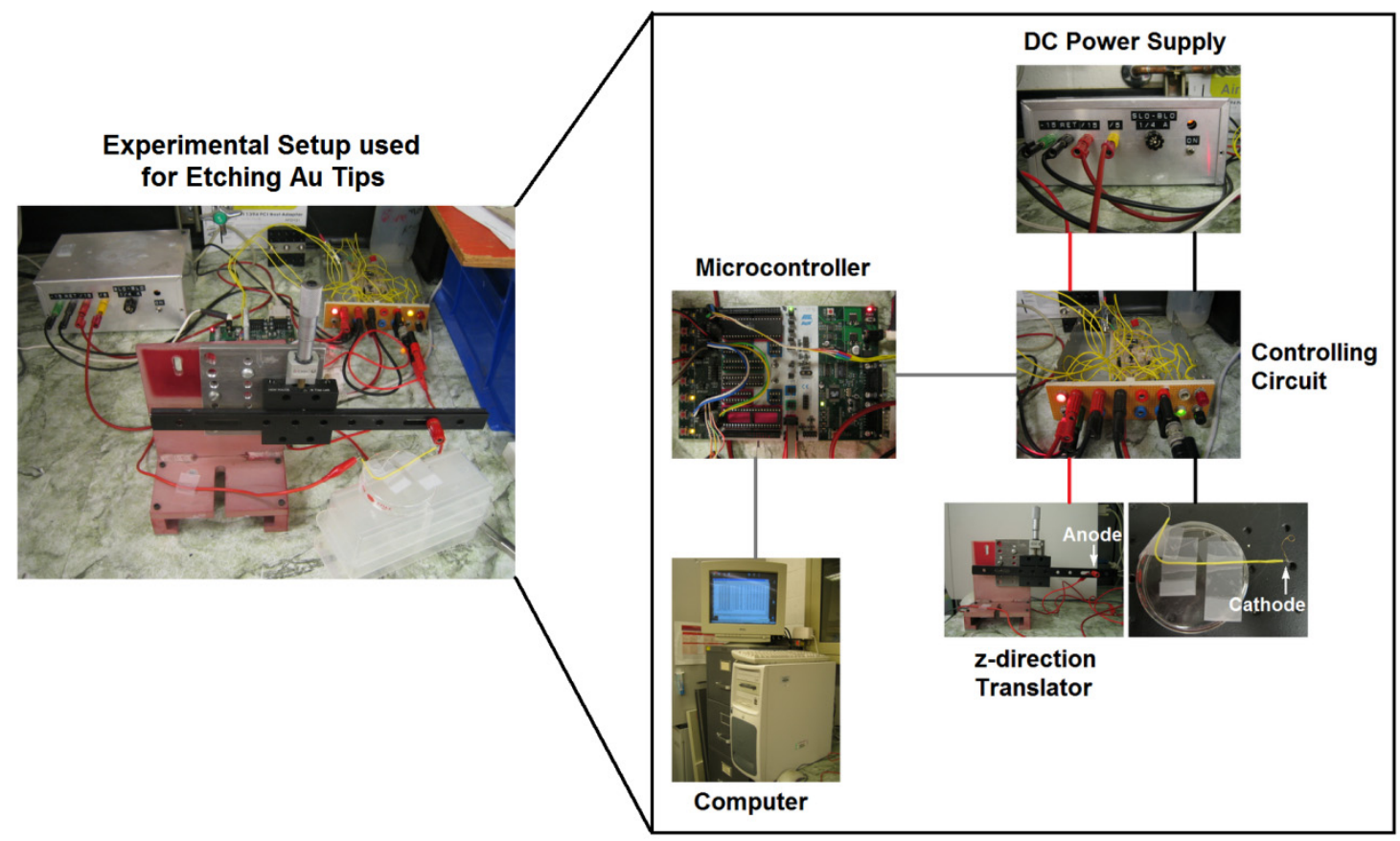

Fig. 3.1.1 Experimental setup used for electrochemically etching gold tips. 


\subsubsection{Results and Discussions}

Gold is a noble metal and as such is extremely stable, with two main oxidation states $\left(\mathrm{Au}^{+}\right.$and $\left.\mathrm{Au}^{3+}\right)$. When an appropriate voltage is applied, gold can be dissolved in a concentrated $\mathrm{HCl}$ solution according to the following ionic reactions: ${ }^{[28]}$

$$
\begin{gathered}
\mathrm{Au}+4 \mathrm{Cl}^{-} \longrightarrow \mathrm{AuCl}_{4}^{-}+3 \mathrm{e}^{-}, \mathrm{E}_{0}=1.002 \mathrm{~V} \\
\mathrm{Au}+2 \mathrm{Cl}^{-} \longrightarrow \mathrm{AuCl}_{2}^{-}+\mathrm{e}^{-}, \mathrm{E}_{0}=1.154 \mathrm{~V} \\
\mathrm{AuCl}_{2}^{-}+2 \mathrm{Cl}^{-} \longrightarrow \mathrm{AuCl}_{4}^{-}+2 \mathrm{e}^{-}, \mathrm{E}_{0}=0.926 \mathrm{~V}
\end{gathered}
$$

Similarly to the etching process of tungsten wires, when the gold wire is immersed into the electrolyte solution, a meniscus forms at the air-liquid interface. As the wire diameter decreases by etching in bulk, the meniscus decreases in height. When the etched part of the wire becomes too thin to keep the lower part of the wire attached, it will break and fall down. At this moment the etching process is self-terminating. The gold ring used as cathode will not be etched during the etching process. However, since deposits will cover the ring surface leading to an electrode of somewhat lower conductivity, the ring has to be cleaned frequently.

In contrast to the etching process of tungsten wires, at a high rate of etching of gold wires, electrochemical reactions mentioned above consume a large amount of $\mathrm{Cl}^{-}$ions near the interface, leading to a depletion of $\mathrm{Cl}^{-}$ions in this region. Subsequently, gold oxides will be formed, which passivate the gold surface, resulting in a significant decrease of the reaction current. However, the fast resupply of $\mathrm{Cl}^{-}$ions from the bulk solution will facilitate the dissolution of gold oxides as $\mathrm{AuCl}_{4}{ }^{-}$so that the bare gold surface exposes to the $\mathrm{HCl}$ acid once more, leading to an increase of reaction current. This process described above occurs again and again, establishing a current oscillation. 
Evidence for this is the periodic change of reaction current during the etching process is shown in Fig. 3.1.2.
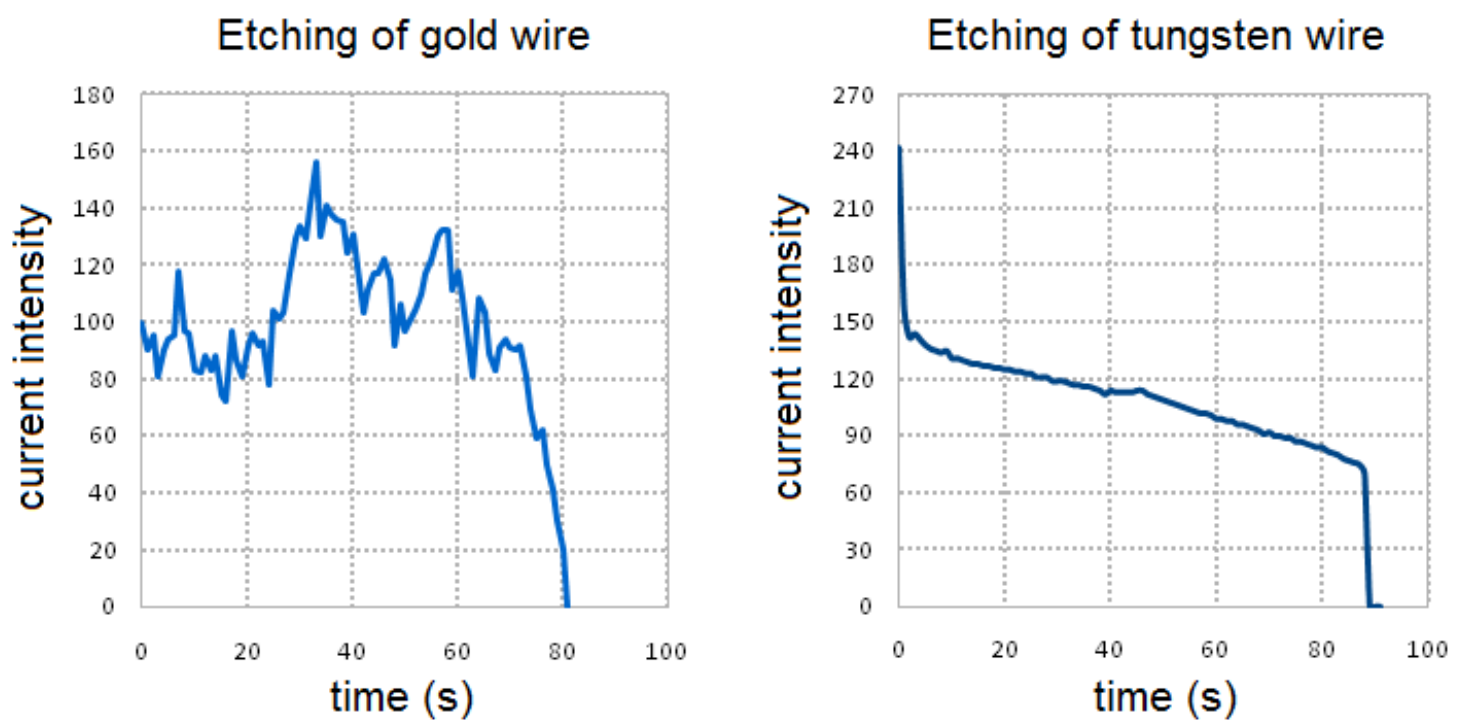

Fig. 3.1.2 Current intensity monitored during different etching processes: (left) etching of gold wire; (right) etching of tungsten wire.

The occurrence of side reactions such as the evolution of $\mathrm{Cl}_{2}$ and $\mathrm{O}_{2}$ causes a severe bubbling effect, which could bring an etching interruption and a rough structure of tip surface. The bubbling effect will be a problem in etching processes and should be reduced as much as possible. Considering the role of ethanol in silicon industry, where ethanol is used to reduce the bubbling and, thereby to produce a smooth tip surface, a certain amount of ethanol is added to the $\mathrm{HCl}$ acid solution to reduce the bubbles produced during the etching process with the aim to obtain gold tips having smooth surfaces. The optimized electrolyte mixture is composed of the hydrochloric acid and ethanol with the proportion 1:1 in volume.

Several parameters can impact the etching process, leading to the etched tips having differences in morphology. These parameters could be the applied dc voltage, the ratio of $\mathrm{HCl}$ acid to ethanol in the electrolyte solution, and the immersion depth of gold wires. 
Herein, the influence of the immersion depth is discussed. By taking account of the fact that cathode of the etching setup is made from the gold wire with $0.2 \mathrm{~mm}$ diameter, 0.15 $\mathrm{mm}, 0.20 \mathrm{~mm}$ and $0.40 \mathrm{~mm}$ were chosen as the immersion depth for gold wires with 76 $\mu \mathrm{m}$ diameters; $0.20 \mathrm{~mm}$ and $0.40 \mathrm{~mm}$ were selected as the immersion depth for gold wires with $100 \mu \mathrm{m}$ diameters. Plots of reaction current collected during the processes for etching $76 \mu \mathrm{m}$ and $100 \mu \mathrm{m}$ gold wires with different immersion depths, as well as optical images of the resulting tips are shown in Fig. 3.1.3 and 3.1.4, respectively. Obviously, a tip with a sharp apex can be obtained when the reaction current is relatively high. And the reaction current could be influenced by the immersion depth. Thus, the optimized immersion depth for the fabrication of gold tips could be: $0.15 \mathrm{~mm}$ for $76 \mu \mathrm{m}$ gold wires and $0.40 \mathrm{~mm}$ for $100 \mu \mathrm{m}$ gold wires. 


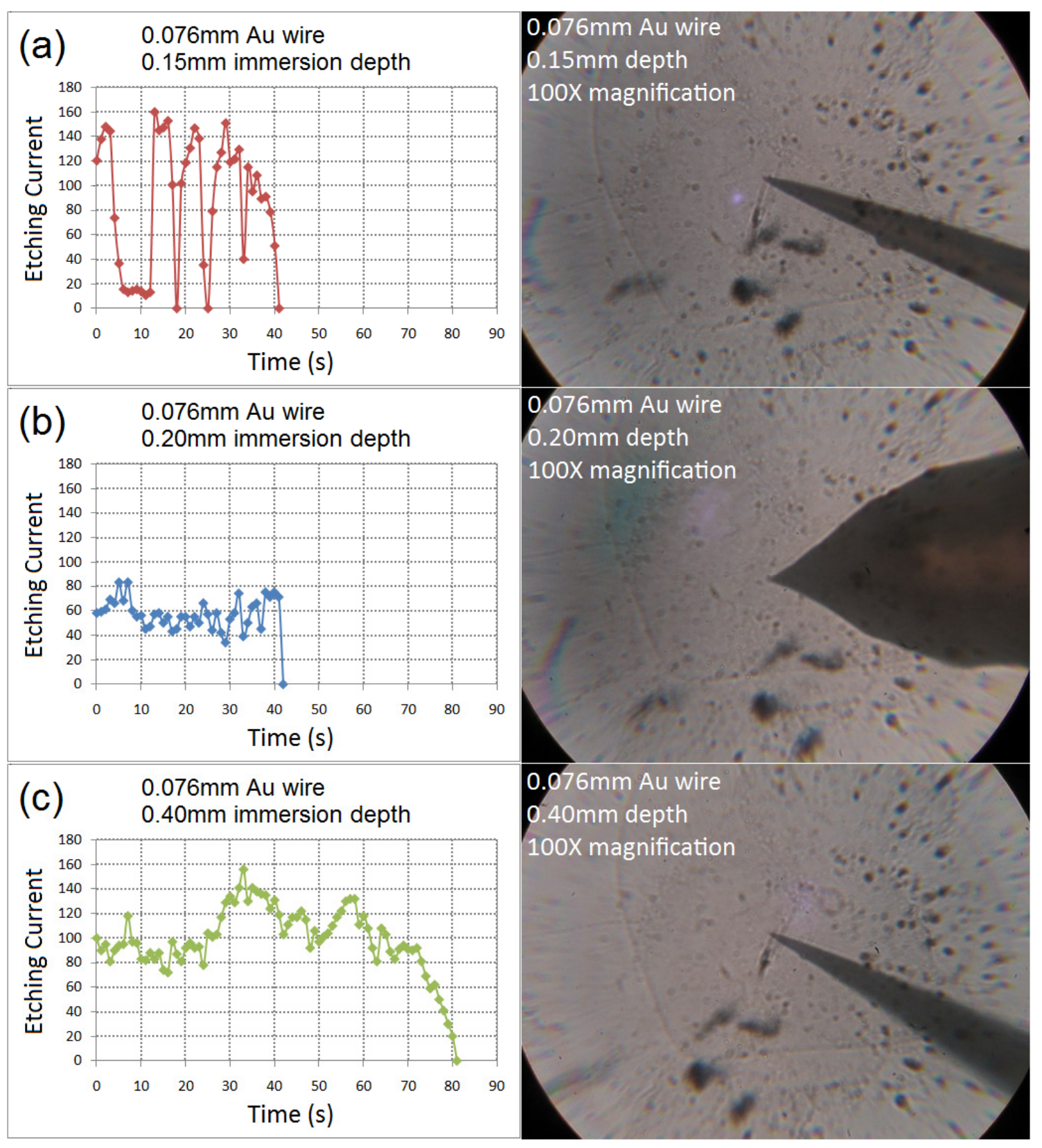

Fig. 3.1.3 Plots of current intensity vs. time for the processes of etching $0.076 \mathrm{~mm}$ diameter gold wires with different immersion depths, and corresponding optical images of resulting tips. 


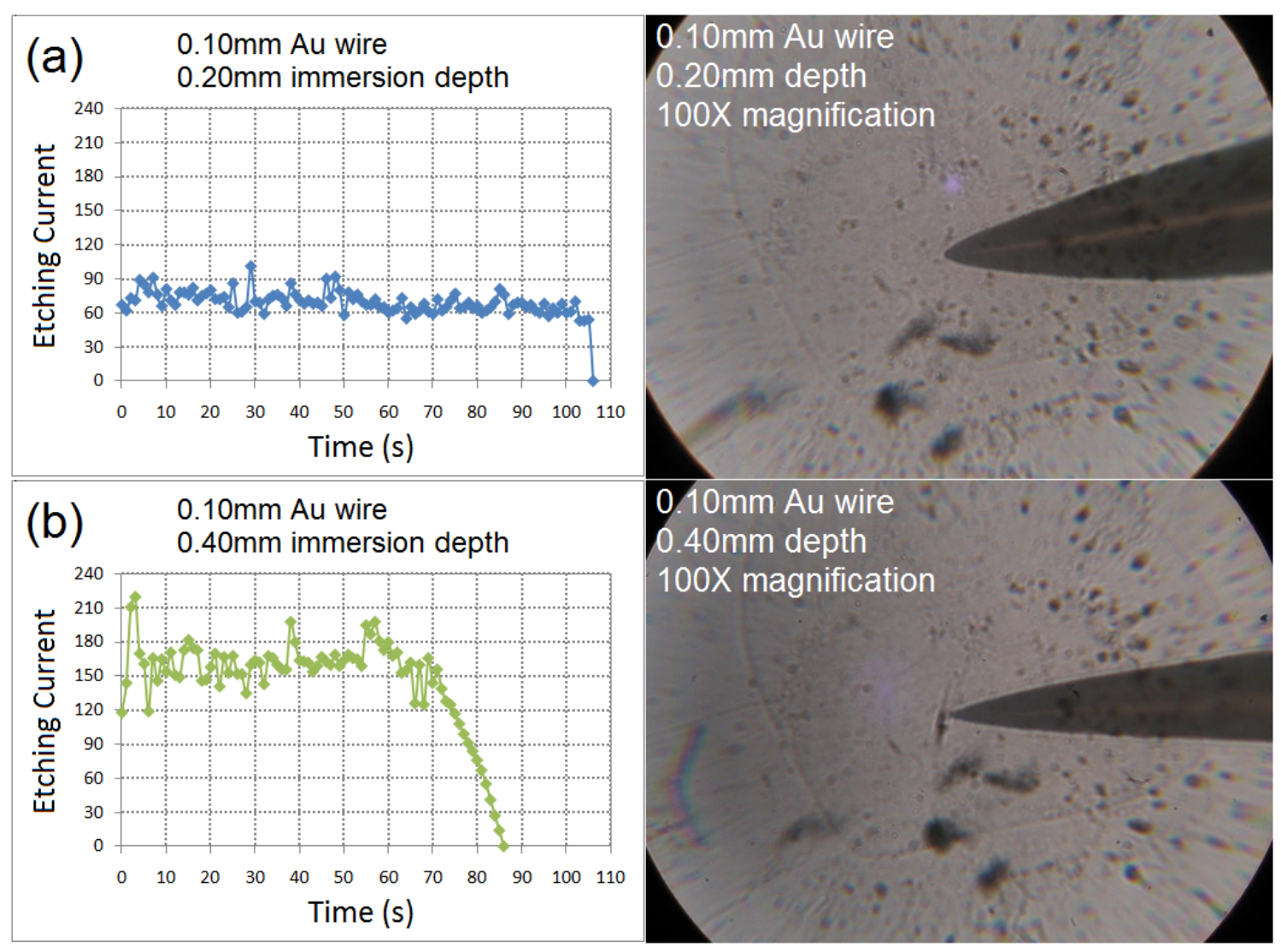

Fig. 3.1.4 Plots of current intensity vs. time for the processes of etching $0.100 \mathrm{~mm}$ diameter gold wires with different immersion depths, and corresponding optical images of resulting tips.

\subsubsection{Conclusions}

In this study, we demonstrate that gold tips can be simply produced by electrochemical etching technique. A thin gold wire is immersed in the etching solution (a mixture of $\mathrm{HCl}$ acid with ethanol having the proportion of 1:1), which is suspended inside a gold ring by surface tension. The gold ring made of a thicker gold wire acts as the counter-electrode. An electric field is applied until the thin wire is completely corroded and a tip is formed. Experimental results show that electrochemical etching can provide gold tips with sufficient quality in a reproducible and reliable manner. 


\subsection{Experimental Instrumentation}

A novel scanning probe microscopy (SPM) system is developed to study the behavior of liquid films confined to nanometer-sized regions as well as other complex surface phenomena. The SPM system includes: the probe, the microscope stage and the detection system. Moreover, the design of this system can be varied depending on the needs of the research.

\subsubsection{The Probe}

Fig. 3.2.1 shows a typical probe implemented in our SPM system which consists of a quartz tuning fork (ESC-.327-12.5-8X, ESC.Inc) and a tapered gold tip attached to one of its tines. The tuning fork (TF), which is usually made of single crystal quartz, has been used as a shear-force detector in a diverse range of fields. ${ }^{[13,29,30]}$ The idea behind such a shear-force detector is to take advantage of the piezoelectric effect native to quartz crystal. When the TF is excited to vibrate in such a way that the tip oscillates parallel to the sample surface, both tines of the TF are piezoelectrically coupled through the metallic contact pads. The geometry of the pads and the coupling between the two tines insure that only one resonant vibration mode of the TF is allowed. This in turn generates an electric signal proportional to the tip oscillation amplitude. When being introduced for distance control, the TF is driven at its resonance frequency. As the tip approaches to the sample surface, probe-sample interactions lead to a damping of vibration amplitude as well as a shift of resonance frequency, thus a resulting reduction of the electric signal amplitude is measured in Fig. 3.2.2 to indicate the tip-sample distance. 


\section{quartz TF}

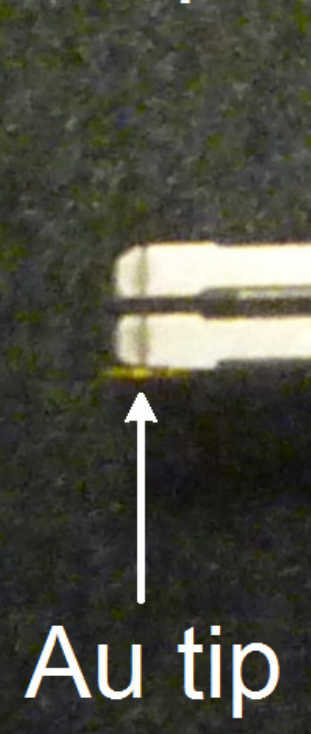

।

\section{$5 \mathrm{~mm}$}

Fig. 3.2.1 A typical probe implemented in our SPM system, which consists of a quartz tuning fork and a tapered gold tip attached to one of its tines.

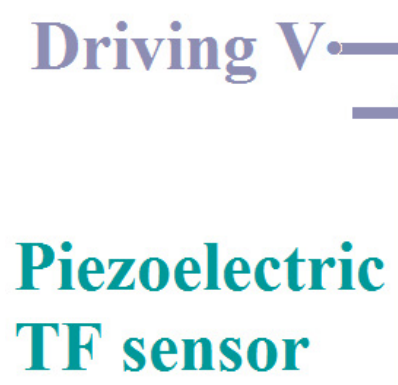

\section{Substrate}

Fig. 3.2.2 TF-based probe-sample distance control. 
Compared to traditional force sensors in dynamic force microscope (i.e., the silicon cantilevers), TFs are much stiffer (the most flexible TF has a spring constant of $k \sim 500 \mathrm{~N} / \mathrm{m}$ ) so that problems concerning the nonlinearity of the oscillator motion in the interaction potential are reduced. The jump-to-contact problem can also be avoided due to the high stiffness. Furthermore, TF's high quality factor Q (100 to 4000 after mounting the tip) can provide a built-in high gain. The implementation of TFs dramatically simplifies the SPMs, making them small, robust and simple to operate compared to optical force measurement systems.

TF is a two-terminal system: one electrical terminal and the other mechanical terminal. To model the electrical terminal, the TF can be equivalent to the ButterworthVan Dyke circuit (Fig. 3.2.3). ${ }^{[15,31]}$ The $C_{p}$ is the real electrical parallel capacitance of TF due to the contact pads and cables. The $L R C$ resonator is the electrical equivalence of the mechanical single harmonic oscillation of TF: the inductance $L$ stands for the kinetic energy storage (i.e., the effective mass); the capacitance $C$ reflects the potential energy storage (i.e., the spring constant); and the resistor $R$ models the dissipative process. Therefore, the electrical admittance measurable through the electrical terminal of TF is given by:

$$
Y(\omega)=\frac{1}{R+i \omega L+\frac{1}{i \omega C}}+i \omega C_{p}
$$

By fitting the admittance, the parameters of $L, R, C$ and $C_{p}$ can be retrieved from the experimental data. The resonance frequency of the LRC resonator is $\omega_{0}=1 / \sqrt{L C}$. 


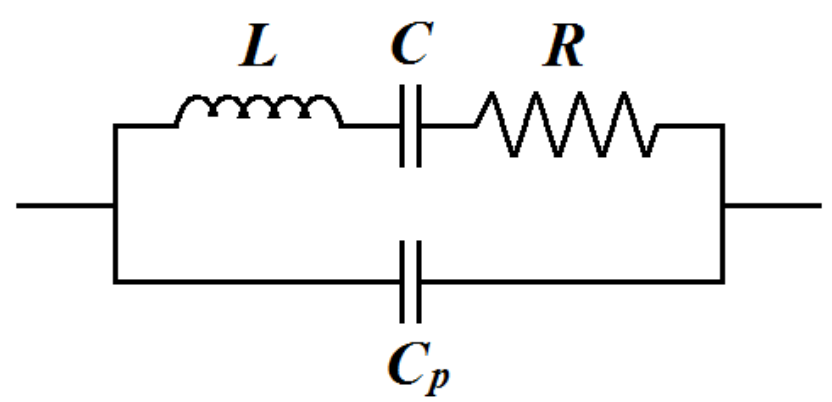

Fig. 3.2.3 The equivalent circuit of the electrical terminal of TF.

To model the mechanical terminal, the TF can be considered as the single harmonic oscillator. ${ }^{[15,32]}$ Two tines of the symmetric TF are doing the damped single harmonic oscillation under the external harmonic force stimulation, which can be described by the Newton equation:

$$
M \stackrel{\mathrm{gg}}{x}=F_{\text {drive }}+F_{\text {damp }}+F_{\text {restore }}=F_{\text {drive }}-M \gamma_{0} \stackrel{\mathrm{g}}{x}-k_{0} x
$$

where $x$ is the displacement of the TF oscillation, $F_{\text {drive }}$ is the driving force, $F_{\text {damp }}$ is the damping force, $F_{\text {restore }}$ is the restoring force due to the TF's elastic deformation, $M$ is the effective mass, $\gamma_{0}$ is the damping rate of free oscillation in air, $k_{0}$ is the spring constant that can be estimated by the formula $k_{0}=(E / 4) w(t / l)^{3}$. Herein, $E$ is the Young's modulus of the material of the tine, $w, t$ and $l$ are the width, thickness and length of the tine, respectively. Then the mechanical response of TF can be written as:

$$
x(\omega)=\frac{F_{\text {drive }} / M}{\omega_{0}^{2}-\omega^{2}+j \gamma_{0} \omega}
$$

in which $\omega_{0}=k_{0} / M$ is the resonance frequency. Thus the amplitude (absolute value) of TF vibration is given by:

$$
A(\omega)=\frac{F_{\text {drive }} / M}{\left[\left(\omega_{0}^{2}-\omega^{2}\right)^{2}+\left(\omega_{0} \omega / Q\right)^{2}\right]^{1 / 2}}
$$


and the phase of TF vibration is written as:

$$
\theta(\omega)=\arctan \left(\frac{\omega_{0} \omega}{Q\left(\omega_{0}^{2}-\omega^{2}\right)}\right)
$$

where is the quality factor of TF.

\subsubsection{The Microscope Stage}

The microscope stage is shown in Fig. 3.2.4. It consists of:

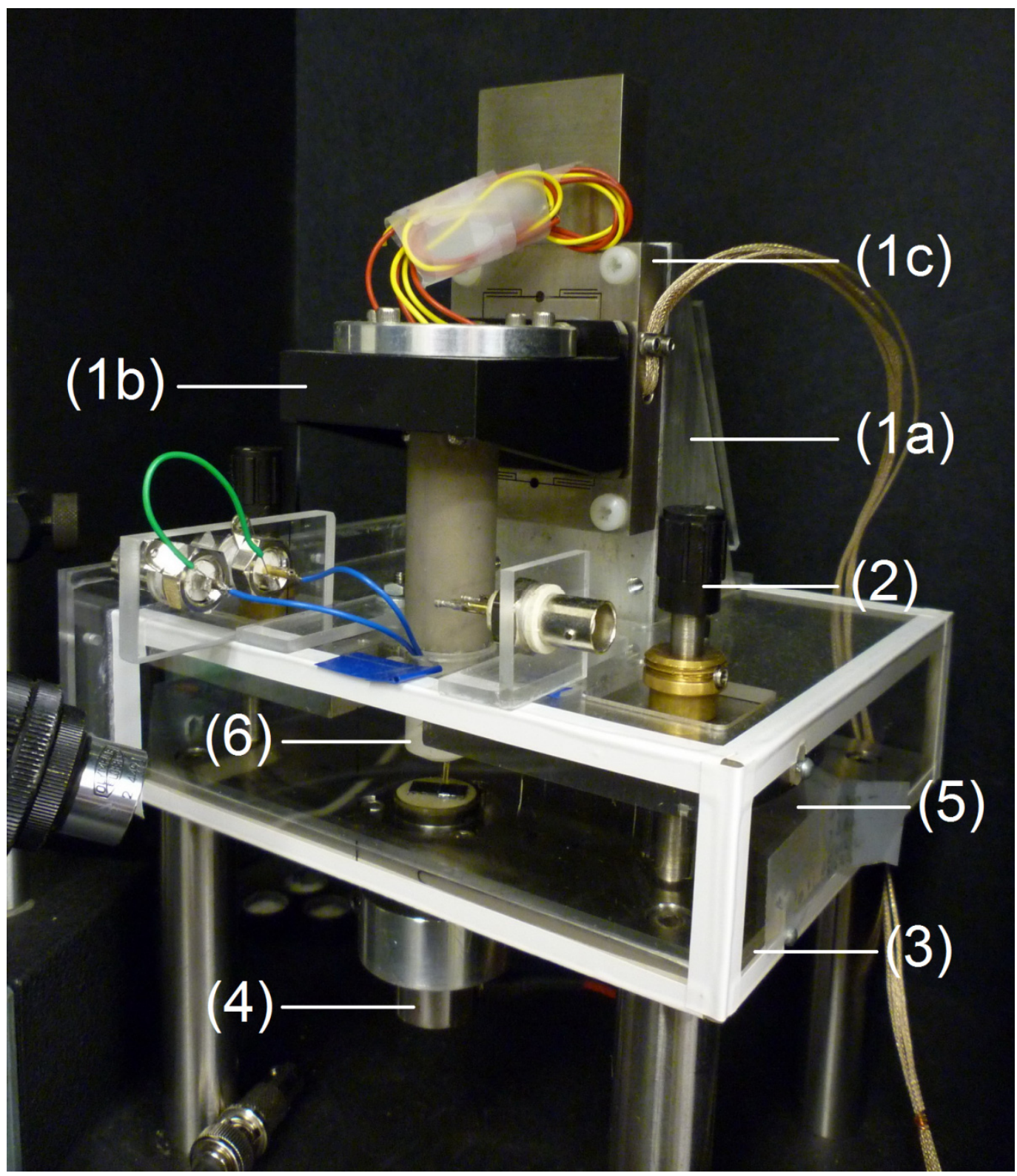

Fig. 3.2.4 The microscope stage. 
(1) The base plate (1a) and the scanner head (1b) where a piezo nanopositioner (NanoOP65, Mad City Labs, Inc.) attached to it. This nanopositioner (1c) allows a fine approach with nanometer accuracy to be achieved.

(2) Three high-resolution screws (AJS100-2, Newport) fixed to the base plate. They allow a coarse approach of the probe to the sample.

(3) The sample stage used to support the scanner head.

(4) The acoustic sensor (SE40-Q, Dunegan Engineering Consultants, Inc) held tightly onto the sample stage.

(5) The humidity chamber made out of acrylic plates.

(6) The TF holder made of plastic and macor.

\subsubsection{The Detection System}

Collections of experimental data are achieved using Stanford Research Systems SR850 and SR844 digital lock-in amplifiers. In a typical experiment, the TF is driven by the sinusoidal voltage from the built-in signal generator of one lock-in SR850 (lock-in $\# 1)$. The same lock-in amplifier is also used to detect the electric response of the TF. Simultaneously, the acoustic sensor is directly hooked up another lock-in SR850 (lock-in \#2) so as to record the acoustic signal generated during experiment. Lock-in \#1 and lockin \#2 are referred to each other by using the same Ref. signal. More details of the detection system will be given in latter chapters. If there are more than two signals that should be measured, the lock-in SR844 is used along with these two SR850 lock-in amplifiers. 


\section{CHAPTER 4. INVESTIGATION OF INTERACTIONS BETWEEN DIFFERENT PROBES AND CHEMICALLY TREATED SAMPLES BASED ON SHEAR- FORCE/ACOUSTIC MEASUREMENTS}

\subsection{Motivations}

Although the shear-force mechanism is widely used in scanning probe microscopes for distance control, its origin is still unclear. Some researchers suggest that the contamination layer (a fluid-like film made out of water and other hydrocarbon molecules) typically found adsorbed on top of a sample surface, plays a significant role for shearforce damping when experiments are performed in ambient conditions. ${ }^{[33,34]}$ In this chapter, the probe-sample interaction will be experimentally investigated as a function of the tip-sample distance based on the approach/retraction measurements taken with our shear-force/acoustic microscope. By systematically changing the experimental conditions, such as the tip geometry, environmental humidity and chemical properties of tip and sample surface, approach/retraction curves may reflect the corresponding variations, hence expressing the influence of contamination layer on the probe-sample interaction.

\subsection{Experimental Section}

A gold tip, fabricated using electrochemical etching described in the previous chapter, is manually glued to one of the tines of a quartz crystal tuning fork (TF). The TF excited at its resonance frequency in a direction lateral to the sample surface, is used to sense the shear-force exerted on the tip. External excitation of the TF is achieved by using a sine wave from the internal reference source of a SR850 lock-in amplifier. The probe's vibration amplitude is estimated by applying the harmonic approximation model, ${ }^{[16]}$ which states $\Delta I=4 \pi f \alpha u_{0}$, where $\Delta I$ is the change in current, $\alpha$ is the 
piezoelectromechanical coupling constant, and $u_{0}$ is the root-mean-square (rms) amplitude of the alternating current generated by the electrically driven TF; at resonance $\Delta I=10 \mathrm{nA} \mathrm{rms}$ gives $u_{0}=4 \mathrm{~nm} \mathrm{rms}$. The sample, a silicon wafer $(1 \mathrm{~cm} \times 1 \mathrm{~cm})$, after an appropriate treatment (i.e., cleaning or chemical modification processes that will be described in the next section), is located on top of an acoustic sensor (SE40-Q, Dunegan Engineering Consultants, Inc.), which is used to detect the acoustic signal generated by the laterally oscillating tip interacting with the sample. In order to improve the quality of acoustic signal, Dow Corning high vacuum grease is used to increase the coupling efficiency of sound wave propagation when the sample bottom surface gets into contact with the acoustic sensor surface.

The experimental setup is schematically presented in Fig. 4.2. This setup can be broken down into two distinct sections: the microscope stage and the detection system. The microscope stage sits on a floating optical bench to minimize mechanical perturbations. A piece of rubber cushion is placed between the stage and the optical bench to further reduce undesigned disturbances. Coarse approach of the probe toward the sample is performed with a set of fine-pitch screws, which allows the adjustment of the tip-sample separation in the range of a few micrometers. During the coarse approach, the tip-sample distance can be monitored through a monitor. When the tip-sample distance is in the range within $50 \mathrm{~m}$, fine approach is performed with a piezo stage (Nano-OP65, Mad City Labs, Inc.) that provides $65 \mu \mathrm{m}$ range of motion in the vertical direction (z-direction) with nanometer accuracy. More importantly, this piezo stage can be operated in close loop so as to eliminate the hysteresis arisen from the piezoelectric meterrial components. 


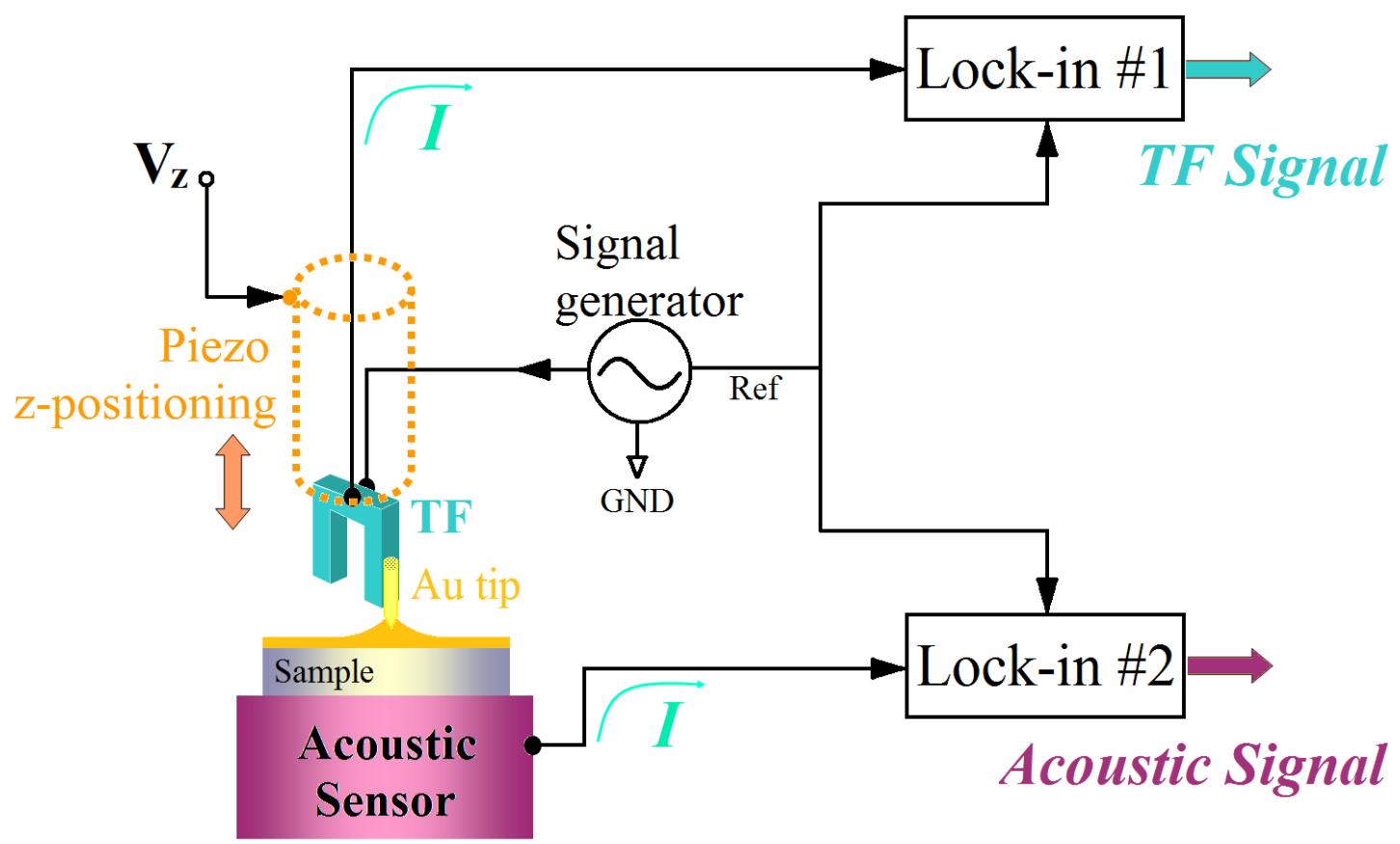

Fig. 4.2 Schematic of the settings used for detecting the tuning fork (TF) signal along with the acoustic signal as the gold tip approaches to, and withdraws from, the sample surface.

With the probe driven at its resonance frequency, approach curves are recorded while approaching the probe to the sample; the acoustic sensor is placed underneath the sample in mechanical contact. This amplitude significantly decreases as the tip approaches some tens of nanometers from the sample surface. The variations of TF signal (as a result of the damping of the tip vibration) and the response of the acoustic sensor are being measured simultaneously by two lock-in amplifiers (SR850, Stanford Research System), both referenced by the same reference signal. Thus, two approach curves are respectively recorded: one for the shear-force probe (TF signal) and one for the acoustic sensor (acoustic signal). To obtain the complete approach/retraction cycle, retraction curves for both $\mathrm{TF}$ and acoustic signals are recorded when retracting the probe from the sample. 


\subsection{Results and Discussions}

The tip-sample interaction that will further influence the measured TF and acoustic signals depends on several parameters, such as the tip geometry, relative humidity of the environment, and the chemical nature of tip and sample surface. The experimental results are presented and discussed below.

\subsubsection{Influence of the Tip Geometry}

The effect of the tip geometry was studied by recording approach/retraction curves on a bare silicon wafer, with two different gold tips when the relative humidity of the environment was controlled to be $25 \%$. Prior to the experimental operation, the silicon wafer was well cleaned using isopropyl alcohol (IPA) with ultrasonic for $15 \mathrm{~min}$ to remove contamination from the surface, and subsequently blown dry by nitrogen. Fig. 4.3.1 shows the scanning electron microscope (SEM) images of a "chubby" tip and a "sharp" tip, respectively. The chubby tip has a large surface of the tip apex, and the corresponding SEM image shows that the diameter is about $700 \mathrm{~nm}$. When the chubby tip was driven to freely vibrate in air, the spectrum obtained by carrying out the frequency sweep displayed a resonance frequency of $f_{\text {res }}=31394 \mathrm{~Hz}$, and a quality factor of $Q=f_{\text {res }} /(\Delta f)_{1 / 2}: 218$. Thus the vibration amplitude of the chubby tip was estimated to be about $8 \mathrm{~nm}$ rms when using a driving voltage of $14 \mathrm{mV}$. The sharp tip with a diameter of about $100 \mathrm{~nm}$ was found to have a resonance frequency of $f_{\text {res }}=31272 \mathrm{~Hz}$, and the quality factor of $Q=f_{\text {res }} /(\Delta f)_{1 / 2}: 279$. By using the same driving voltage $(14 \mathrm{mV})$, the sharp tip was excited to freely oscillate in air with an amplitude of about $9 \mathrm{~nm}$ rms. Approach curves of the TF and acoustic signals were simultaneously recorded while the 
tip moved towards the cleaned silicon surface. Similarly, the response for both TF and acoustic sensor were recorded as retraction curves when the tip released from the sample.
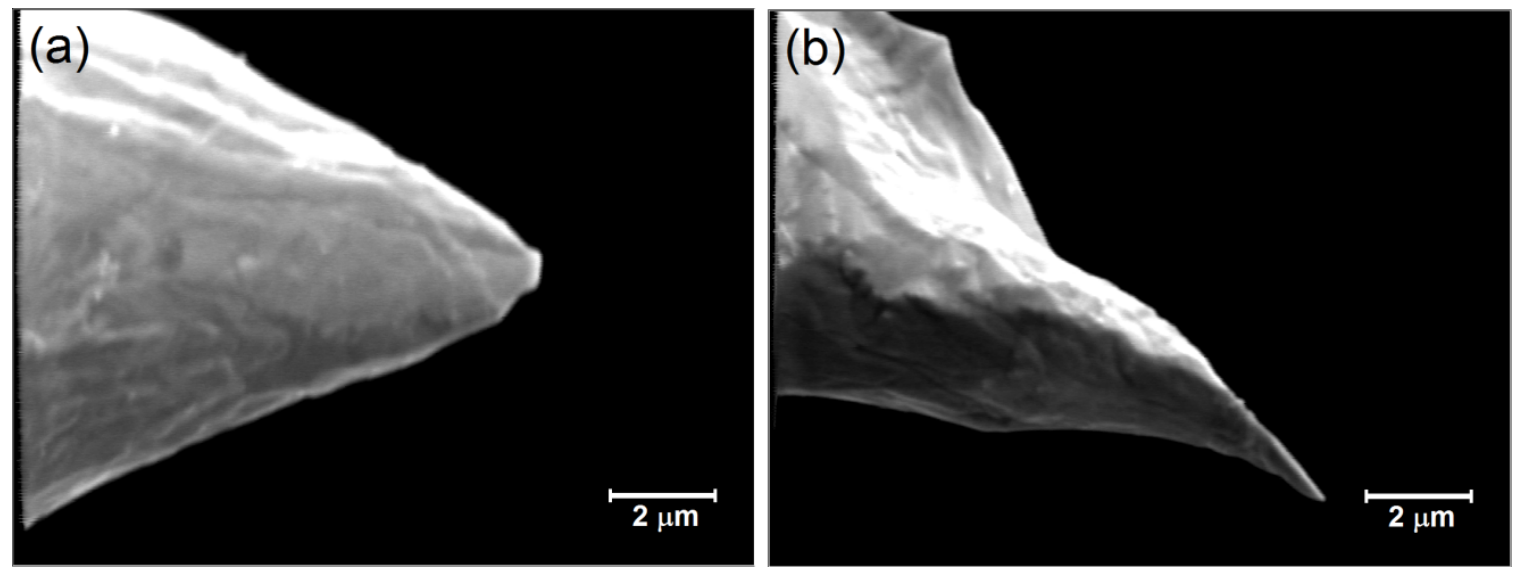

Fig. 4.3.1 Scanning electronic images of (a) a "chubby" gold tip and (b) a "sharp" gold tip.

Fig. 4.3.2 shows the change of $\mathrm{TF}$ and acoustic signals separately as a function of the relative vertical position (distance $\mathrm{z}$ ) of the chubby tip during one approach/retraction cycle. As the tip-sample separation was reduced by bring the tip closer to the sample, the effect of damping forces acting on the probe was observed. The position where the TF signal starts to drop is arbitrarily set to be the zero position. As shown in the plot of TF signal, when approaching the sample surface the tip experiences a sudden increase in damping forces which causes an amplitude decrease of $15 \%$, as indicated at point $\mathrm{A}$ in Fig. 4.3.2a. This amplitude change could be attributed to the presence of a water meniscus. ${ }^{[35]}$ This meniscus is formed when the tip enters into a surface "contamination" layer, which consists of water moleculaes adsorbed from the ambient. On further approach the tip's amplitude (TF signal) is observed to decrease monotonically. It could be explained by the fluid-like layer on top of the solid surface that induces viscous damping of the vibration behavior reflecting in an amplitude decrease. ${ }^{[36]}$ Usually, the 
approach/retraction cycle presents hysteresis (not due to the z-positionor, which has already been corrected, but to the nature of the interaction). However, from $0 \mathrm{~nm}$ to 20 $\mathrm{nm}$ of the plots in Fig. 4.3.2, the difference between approach and retraction curves are not signidificant. The jump displayed by the TF signal at the end of the retraction curve (point B in Fig. 4.3.2a) indicates the tip is detaching from the boundary of the contamination layer. 


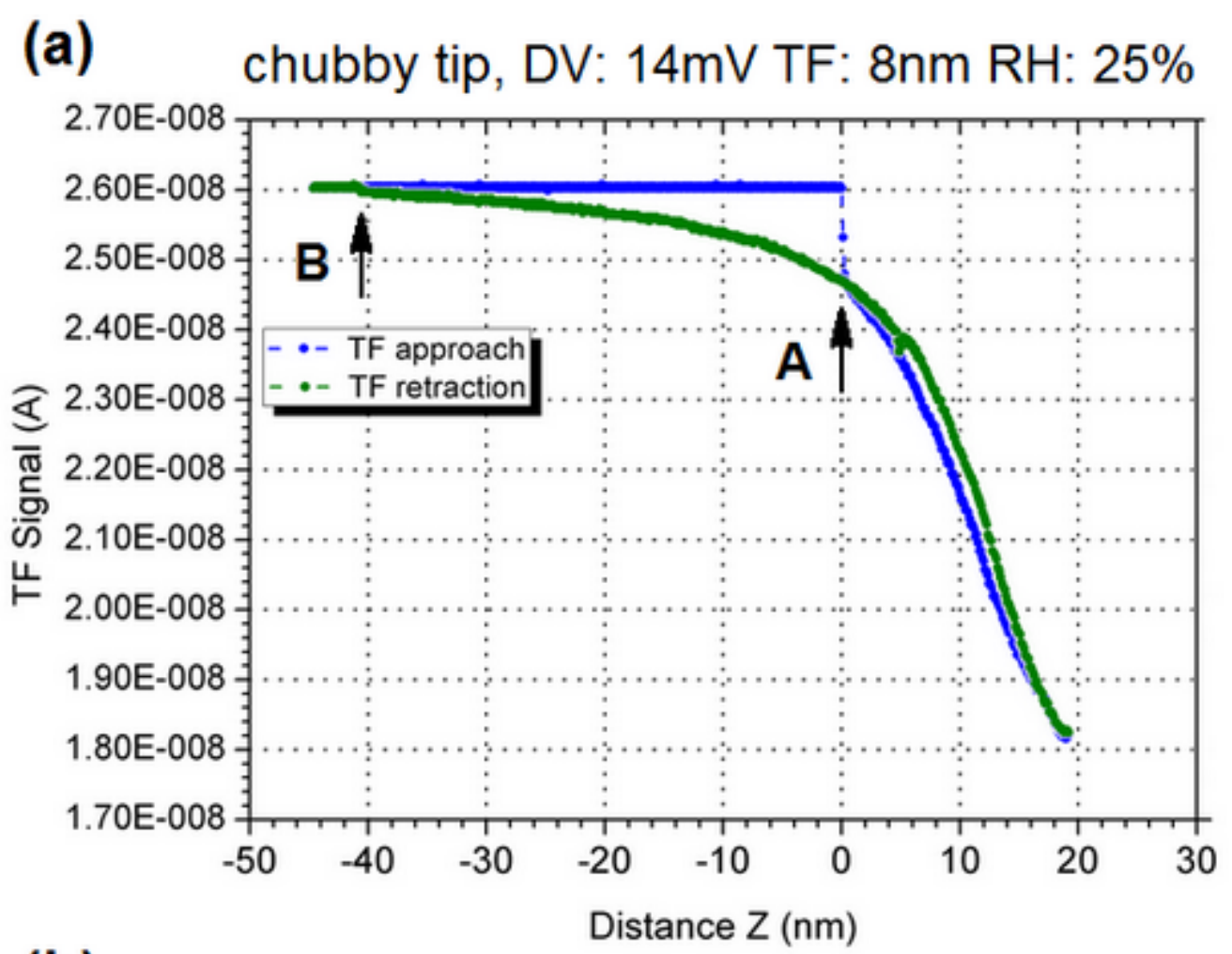

(b)

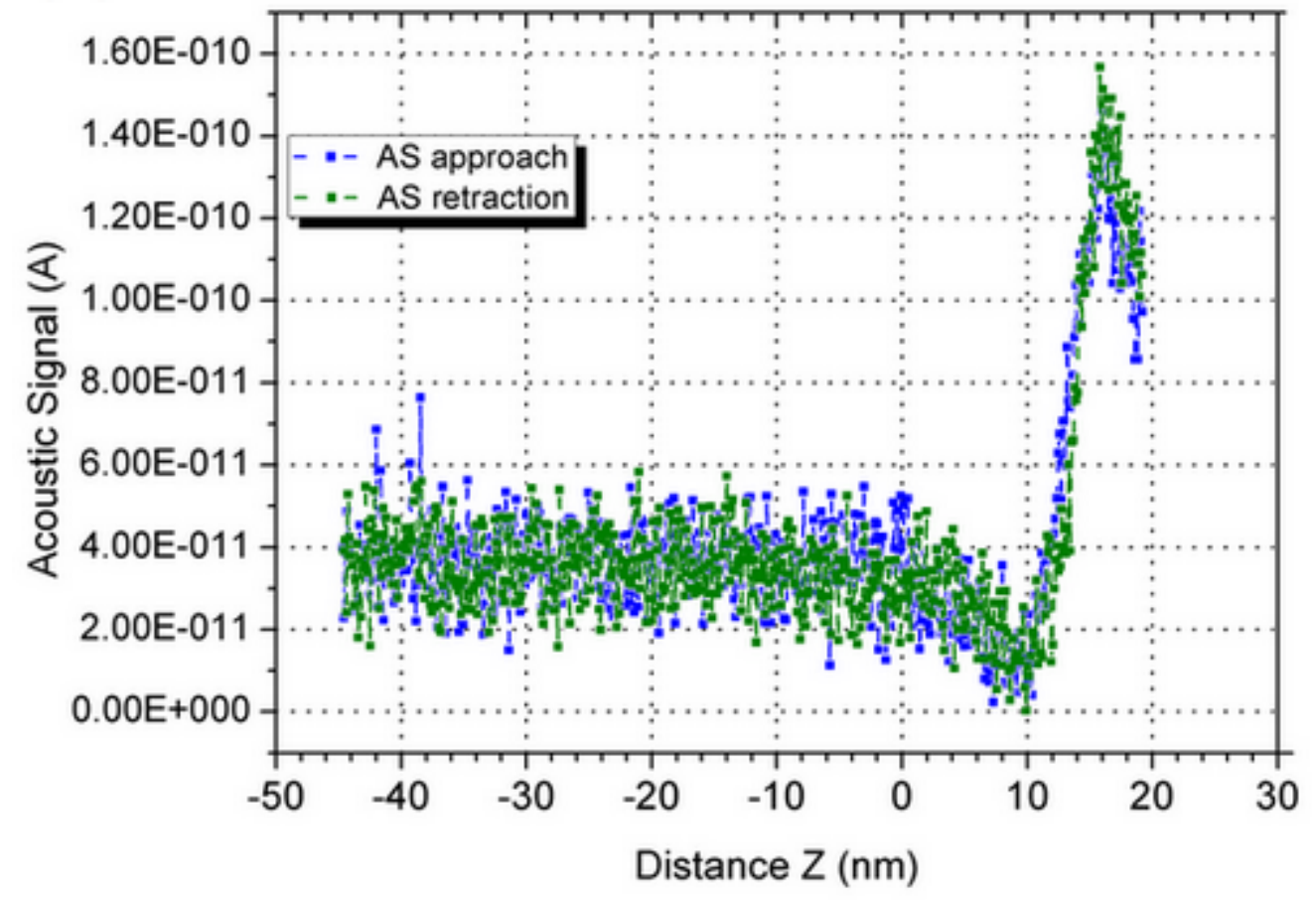

Fig. 4.3.2 Approach and retraction curves of (a) TF and (b) acoustic signals obtained using the chubby tip (humidity: 25\%). 
More information with regard to the interaction of the tip with the contamination lay or/and the sample surface can be gained from the simultaneously obtained acoustic signal. After combining the curves of TF and acoustic signals, changes of these two signals as a function of the tip-sample distance are shown in Fig. 4.3.3 and Fig. 4.3.4, corresponding to the approach and retraction process, respectively. Concerning the curves in Fig. 4.3.3, there are three different zones in the approach: (1) A zone of free oscillation, in which the tip vibrates at its initial amplitude and generates a standing wave of sound received by the sensor as the non-zero acoustic signal. (2) A transition zone, where damping takes place and changes in acoustic signal is sensed. Moreover, this zone can be divided to two pieces: a region (a) where a positive correlation between TF and acoustic signals exists; and the other region (b) in which the correlation between these two signals becomes negative. In region (a), the acoustic signal decreases as the TF's amplitude is damped, indicating that the correlation of these two signals is positive. This could be the result of the interaction between the tip and the contamination layer when the tip entering into it. Due to the larger surface area of the chubby tip, the vibration of tip is restricted by the liquid layer. Additionally, top layers of the liquid maybe not dense enough so that the viscosity is not sufficient to generate sound. Altogether, less sound could be created and subsequently detected by the sensor. In region (b), the acoustic signal increases with decreasing the TF signal. The negative correlation between these two signals could result from the distance-dependent viscosity of the liquid layer, as well as the partial contact of the tip with the solid sample surface. (3) Finally, a contact zone that corresponds to the interaction between the tip and the sample occurs. The vibration of tip is stopped by the mechanical contact, leading to the decrease in acoustic signal. A similar analysis for the 
experimental data can be applied to the retraction curves of TF and acoustic signals shown in Fig. 4.3.4 as well. Details will not be repeated herein.

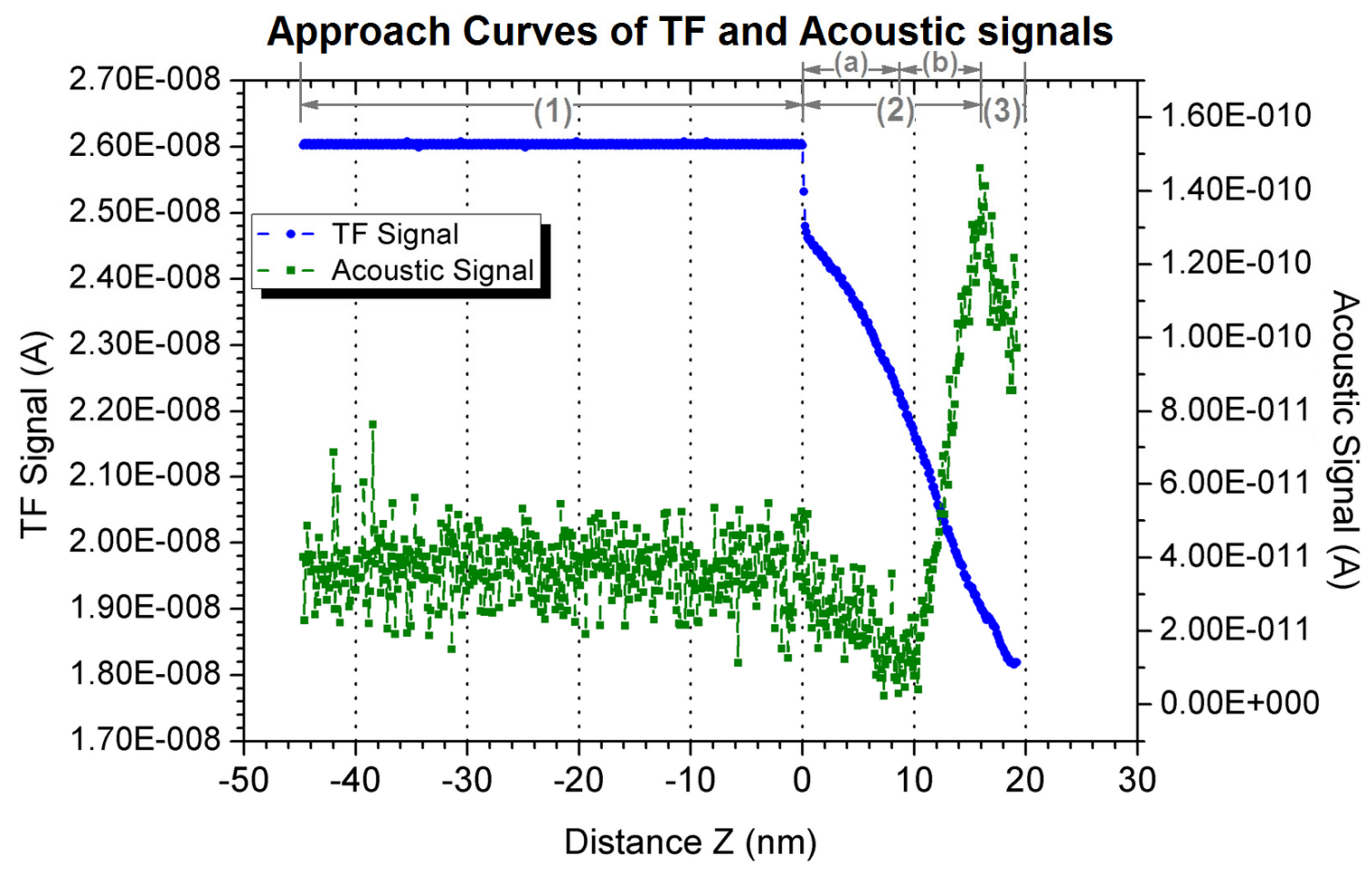

Fig. 4.3.3 Dependences of simultaneously measured TF damping and the acoustic response on the tip-sample distance during the approach process of the chubby tip. 


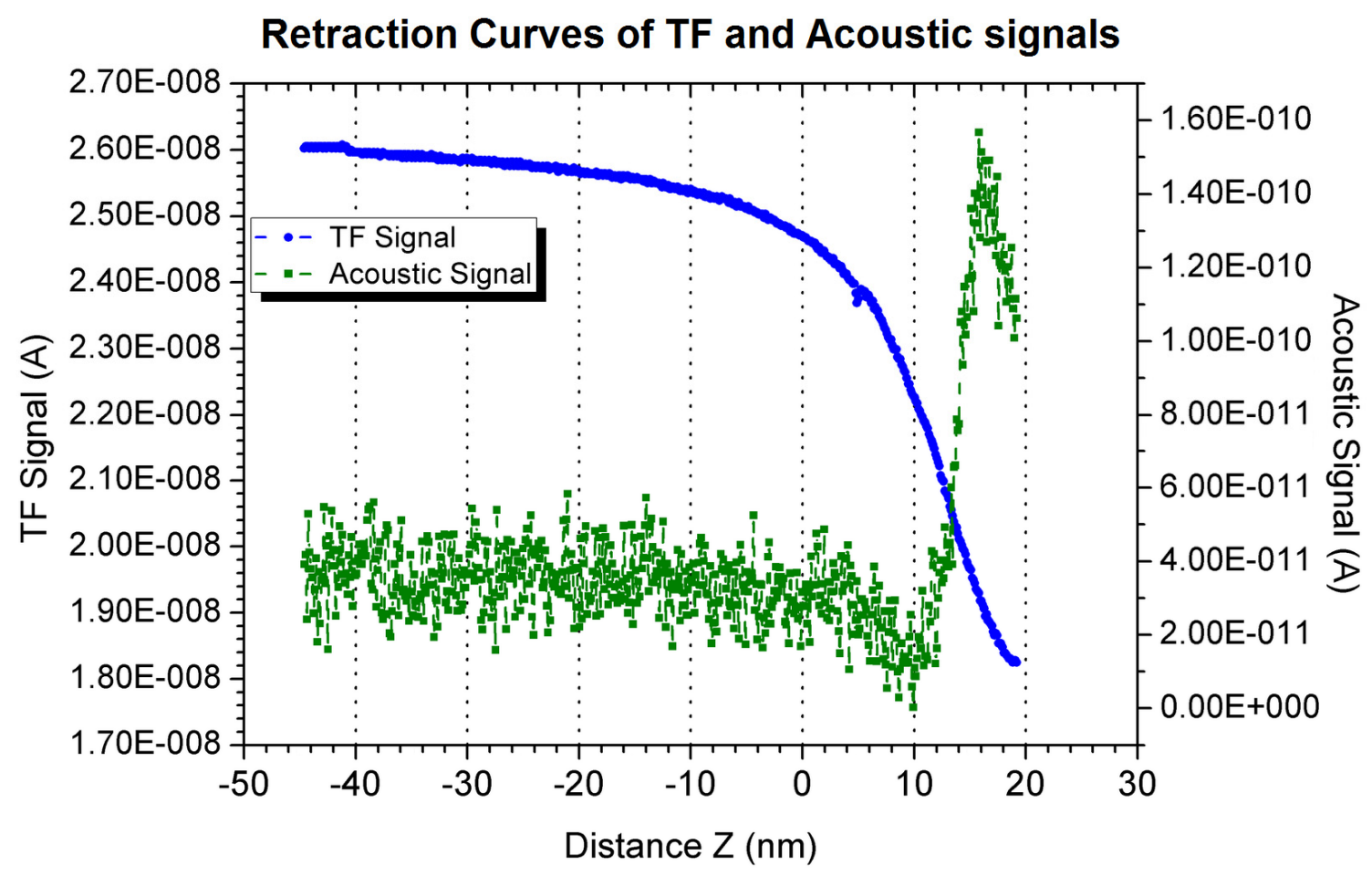

Fig. 4.3.4 Dependences of simultaneously measured TF damping and the acoustic response on the tip-sample distance during the retraction process of the chubby tip.

When the chubby tip was replaced by the sharp tip, approach/retraction curves obtained by performing the same experiment described above are shown in Fig. 4.3.5, indicating that the TF and acoustic signal vary as a function of tip-sample separation. Compared to the sudden jump at point A in Fig. 4.3.2a, the jump displayed on the TF's approach curve (point A in Fig. 4.3.5a) is not noticeable. This could be attributed to the smaller surface area of the sharp tip. When withdrawing the sharp tip from the sample, the tip travels a shorter distance until completely leave the liquid layer (point B in Fig. 4.3.5a). This suggests that the tip-sample interaction becomes weaker due to the surface area of tip getting smaller. 

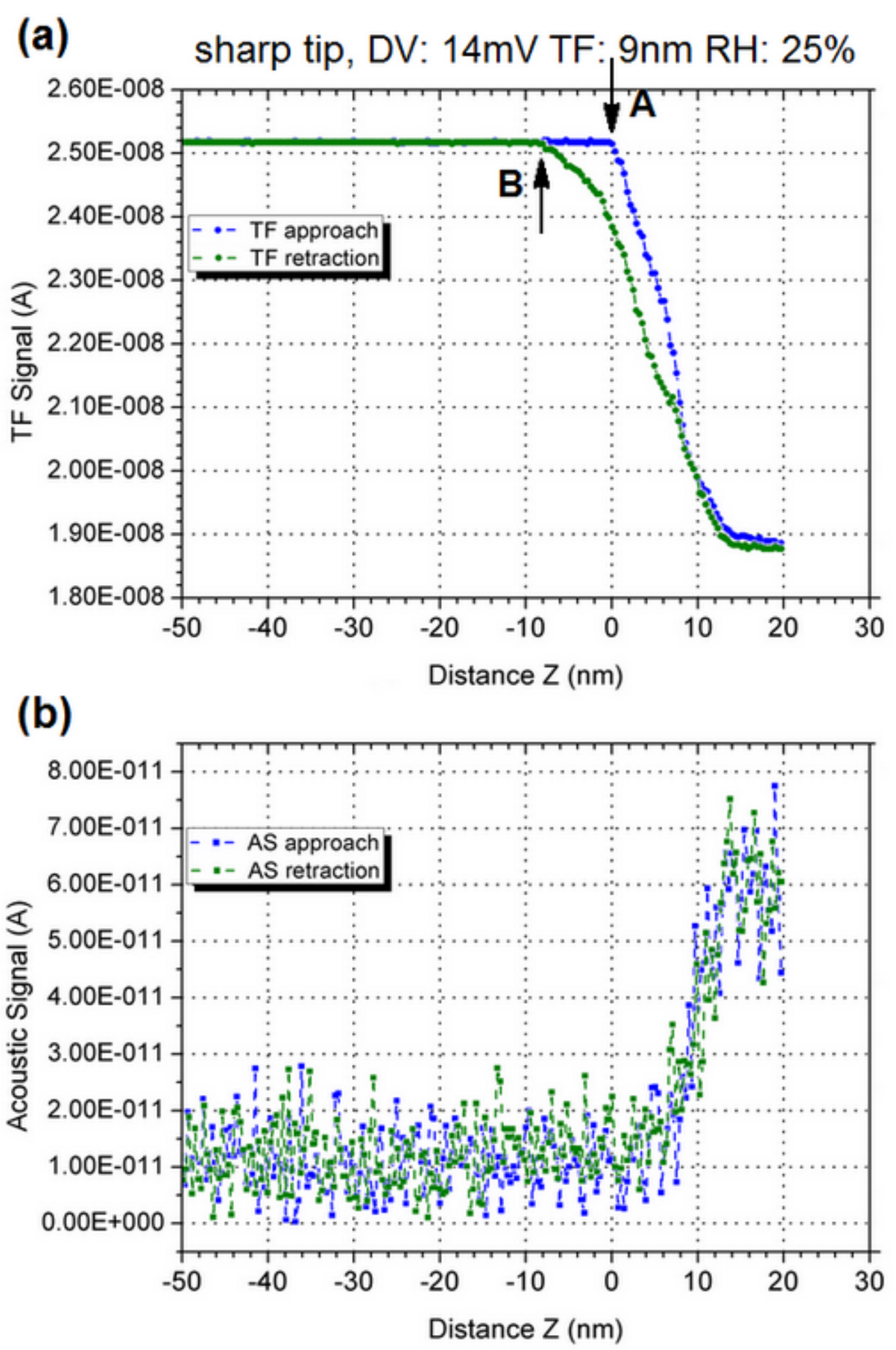

Fig. 4.3.5 Approach and retraction curves of (a) TF and (b) acoustic signals obtained using the sharp tip (humidity: 25\%). 
Fig. 4.3.6 shows the TF signal along with the acoustic signal during the approach. As discussed above, this process can be broken down into three different regions: (1) A region of free oscillation; (2) a damping region; and (3) a contact region. Differing from what we obtained using the chubby tip, there is no positive correlation between the TF and acoustic signals displayed in region (2) if the tip was replaced by the sharp tip. The increase of the acoustic signal in this damping region could result from the dissipative and conservative tip-sample (solid/liquid or solid/liquid + solid) interaction. ${ }^{[15,16]}$ However, the origin of this interaction is not clear, it can be attributed but not limited to a combination of many forces such as the electrostatic forces, van der Waals forces, viscous forces, etc. Curves of TF signal associated with acoustic signal obtained by taking the sharp tip away from the sample are plotted in Fig. 4.3.7.

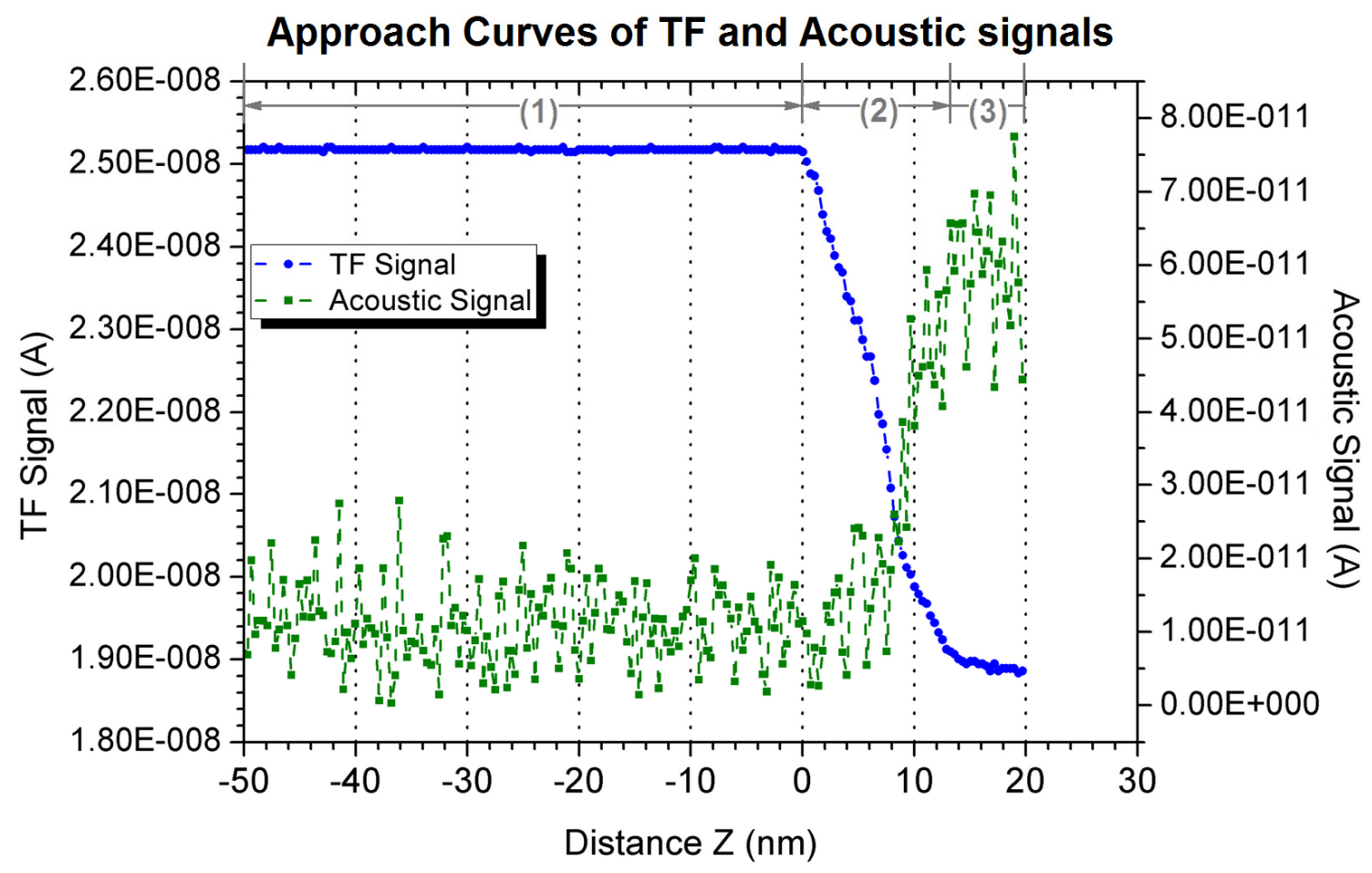

Fig. 4.3.6 Dependences of simultaneously measured TF damping and the acoustic response on the tip-sample distance during the approach process of the sharp tip. 


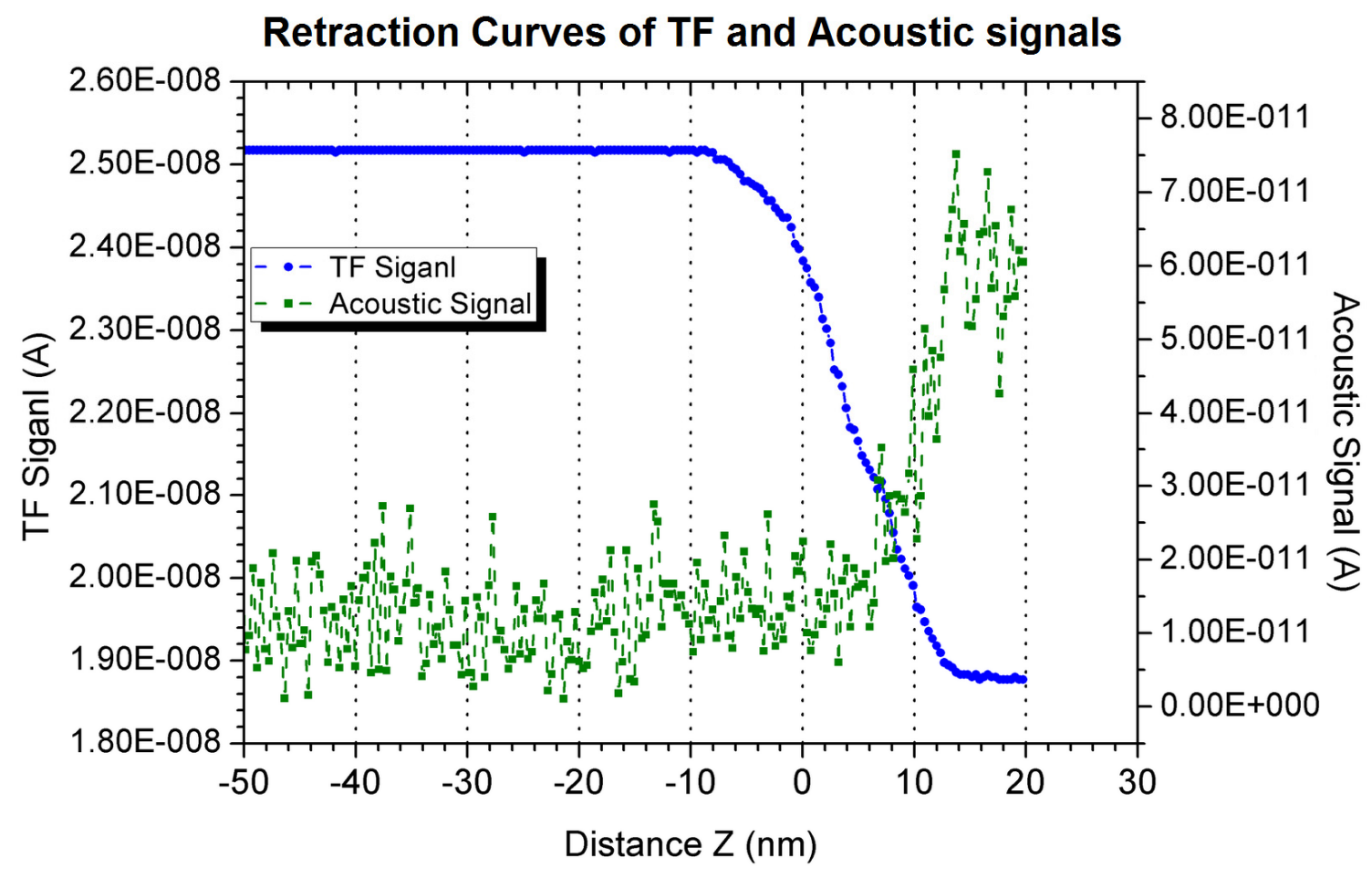

Fig. 4.3.7 Dependences of simultaneously measured TF damping and the acoustic response on the tip-sample distance during the retraction process of the sharp tip.

\subsubsection{Influence of Environmental Humidity}

The effect of relative humidity $(\mathrm{RH})$ in ambient was investigated by performing the same experiments as described above when increasing the humidity of the environment to $55 \%$. This was achieved by simply using a saturated salt solution in a small humidity chamber specifically designed and built for this purpose. Fig. 4.3.8 shows approach/retraction curves of TF and acoustic signals recorded as a function of tipsample distance using the same chubby tip for studying the effect of tip geometry. The zero distance between the tip and sample is defined at the point where the damping of TF signal occurs. 

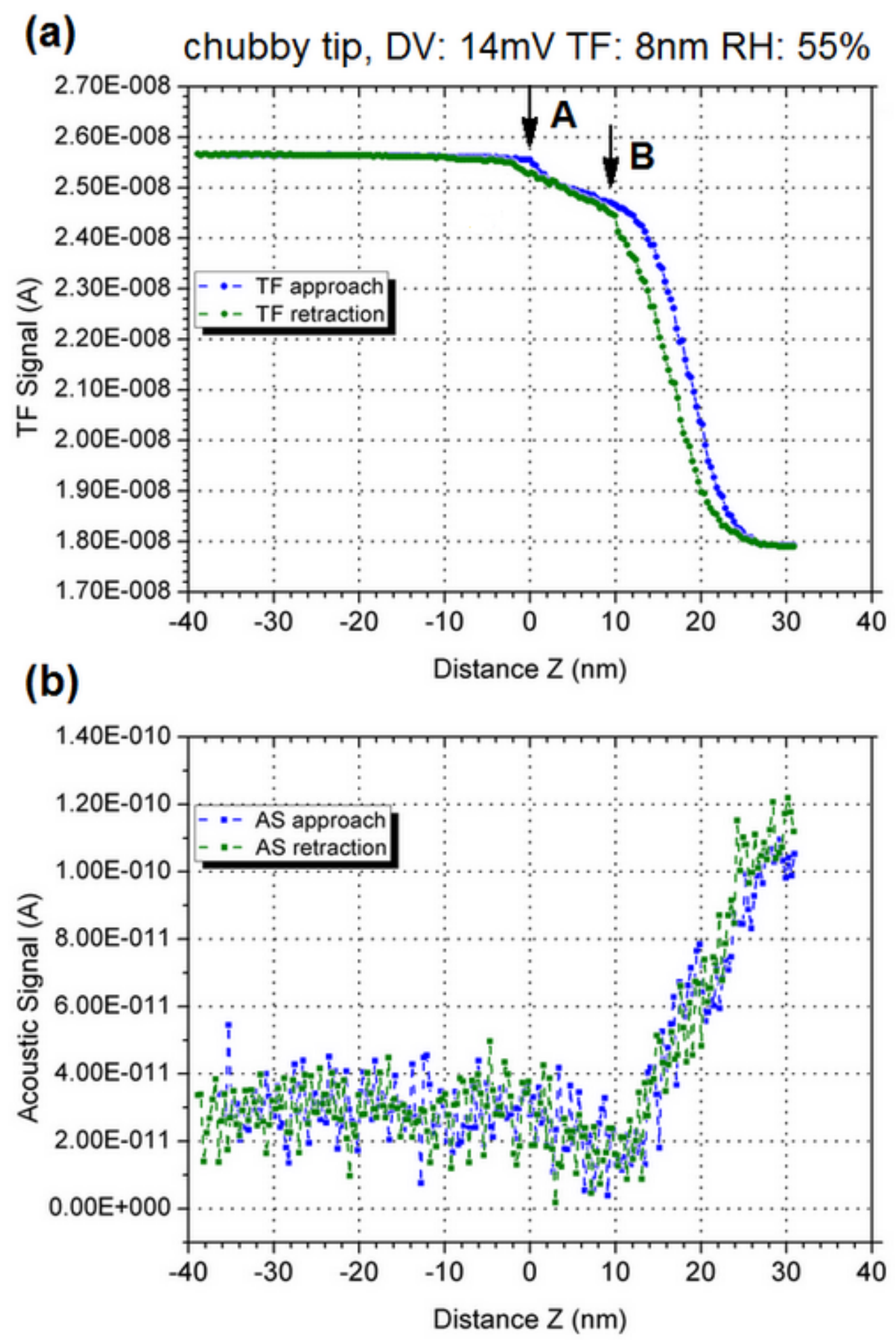

Fig. 4.3.8 Approach and retraction curves of (a) TF and (b) acoustic signals obtained using the chubby tip (humidity: 55\%). 
As the humidity increases to $55 \%$, the approach curve of TF signal exhibits a slow decay region located between point A and B in Fig. 4.3.8a. By contrast, this slow varying part was not evident when humidity was relatively low (25\%). This difference could be explained by the growth of the adsorbed water layer, since the thickness of the water film on the sample depends on the humidity. This result is in agreement with the conclusion drawn in near-field scanning optical microscopy where similar experiments have been carried out. ${ }^{[33,37,38]}$ Additionally, due to the presence of mobile charges in the tip and surrounding, ${ }^{[39]}$ the electrostatic interaction between probe and sample may become more significant when using a chubby tip at low humidity. Therefore, the tip has to retract longer distant from the sample to come back to free oscillation if the electrostatic interaction is stronger. This could explain the longer retraction curve obtained at lower humidity in our experiments.

The approach/retraction curves of TF and acoustic signals were also obtained at humidity of 55\% for the same sharp tip used before. The results are plotted in Fig. 4.3.9. Although the slow decay region is not as obvious as the one that exhibits in Fig. 4.3.8a, an elongation of the overall approach curve is found (lengths of approach curve: about 20 $\mathrm{nm}$ obtained at low humidity, and about $26 \mathrm{~nm}$ obtained at high humidity) because of the growth of water film. 

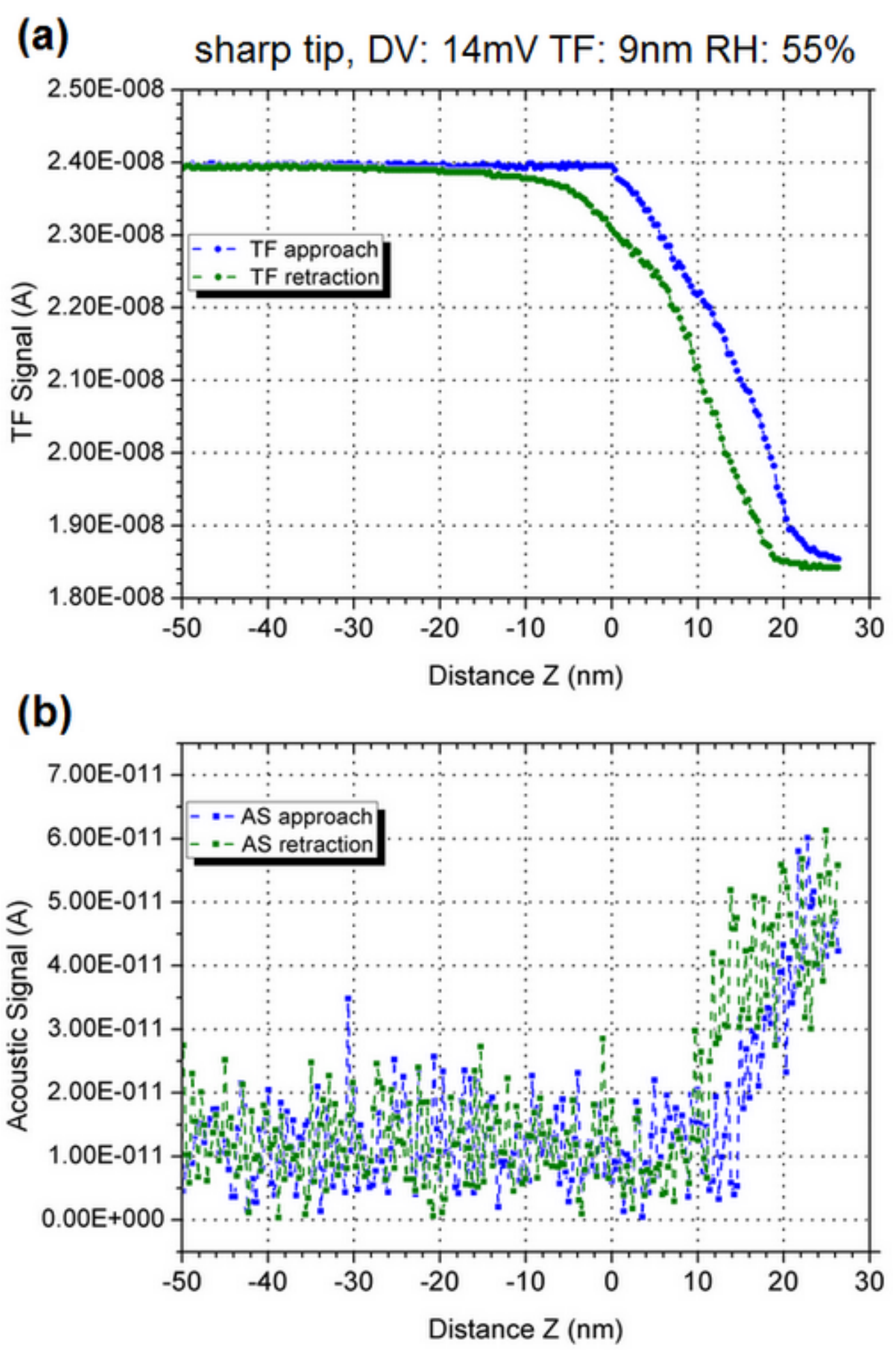

Fig. 4.3.9 Approach and retraction curves of (a) TF and (b) acoustic signals obtained using the sharp tip (humidity: 55\%). 


\subsubsection{Influence of chemical affinity of sample surface}

The chemical affinity of the sample surface was modified to study probe-sample interactions with different types of contamination layers. Specific water affinity was obtained by deposition of polymer monolayers. ${ }^{[40,41]}$ The bare silicon wafer was first cleaned by immersion in piranha solution $\left(35 \% \mathrm{H}_{2} \mathrm{O}_{2} / \mathrm{H}_{2} \mathrm{SO}_{4}=3: 7(\mathrm{v} / \mathrm{v})\right)$ at $80-90^{\circ} \mathrm{C}$ for $60 \mathrm{~min}$ and was washed with boiling water for $90 \mathrm{~min}$, dried under $\mathrm{N}_{2}$. The well cleaned silicon wafer was then chemically functionalized with perfluorophenylazides (PFPA)silane to introduce a monolayer of azido groups on the substrate surface. A $10 \mathrm{mg} / \mathrm{ml}$ solution of polystyrene (PS) in toluene was spin coated on the treated water.

Subsequently, the PS-coated substrate was irradiated under a $450 \mathrm{~W}$ medium-pressure mercury lamp with $280 \mathrm{~nm}$ filter for 5-10 min. One half of the sample was covered by aluminum foil, and only the half without aluminum foil coverage was exposed to the UV irradiation. After removal of unbound PS by solvent extraction, a PS thin film (about 7 $\mathrm{nm}$ thick) cover a half of wafer. By replacing the PS solution with a $10 \mathrm{mg} / \mathrm{ml}$ solution of poly(2-ethyl-2-oxazoline) (PEOX) in chloroform, the same process described above can be used for obtaining a PEOX thin film (having approximately same thickness as the PS film) on the inactivated substrate surface. Fig. 4.3.10 shows the experimental procedure for the chemical treatment. 


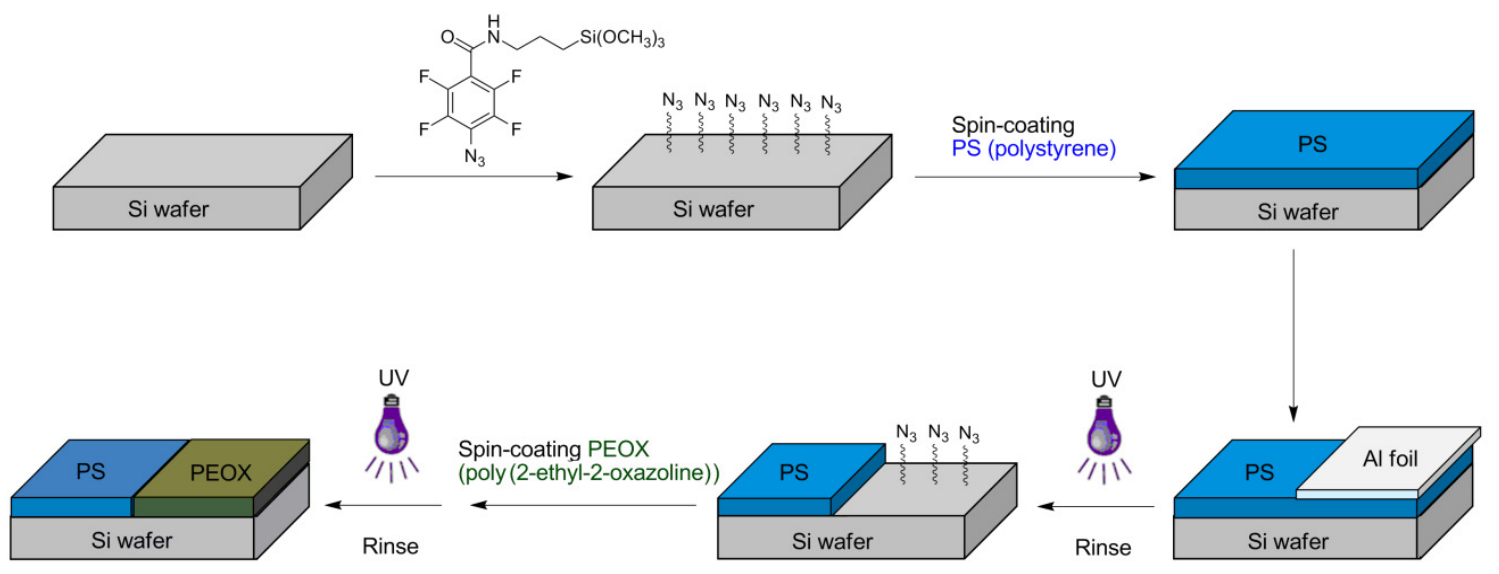

Fig. 4.3.10 Scheme of the experimental procedure for preparing hydrophobic and hydrophilic surfaces on the same silicon substrate.

Water affinity of the sample surface coated with different polymer films can be characterized by performing contact angle measurements. Results displayed in Fig. 4.3.11 show that one half of the substrate covered by PS monolayer is hydrophobic (contact angle is about $88^{\circ}$ ), while the other half coated with PEOX monolayer is hydrophilic (contact angle is about $40^{\circ}$ ).
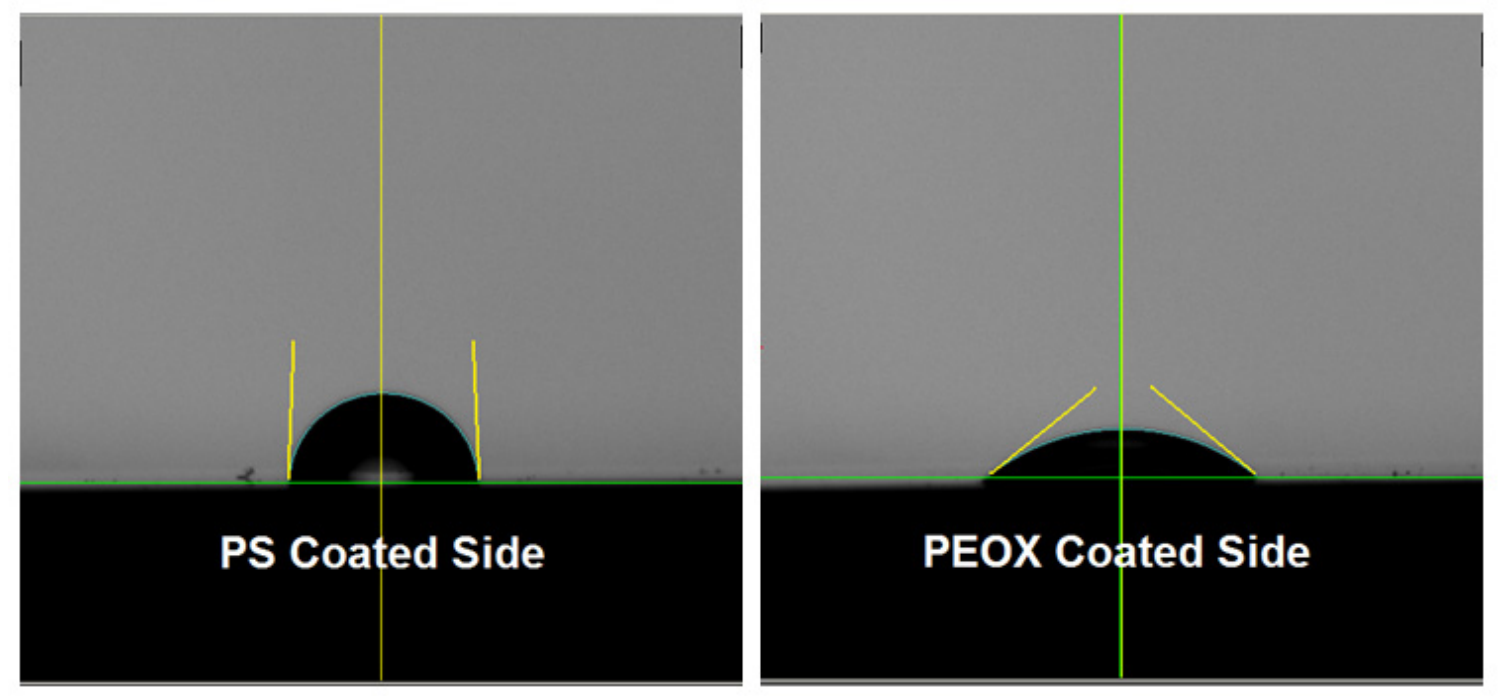

Fig. 4.3.11 Results of contact angle measurements of the silicon substrate covered by PS and PEOX monolayers, respectively. 
The tapered gold tips were chemically functionalized as well. When the PS-coated gold tip was driven to freely vibrate in air, the spectrum obtained by carrying out the frequency sweep displayed a resonance frequency of $f_{\text {res }}=31598 \mathrm{~Hz}$, and the quality factor of $Q=f_{\text {res }} /(\Delta f)_{1 / 2}: 195$. Thus the vibration amplitude was estimated to be about 9 $\mathrm{nm}$ rms when using a driving voltage of $16 \mathrm{mV}$. The approach/retraction curves of $\mathrm{TF}$ signal as well as acoustic signal are shown in Fig. 4.3.12 and Fig. 4.3.13, figures correspond to the experimental data recorded with the hydrophobic surface and hydrophilic surface, respectively.

Curves in both hydrophobic and hydrophilic cases present the same trend of the acoustic signal beginning to increase as a decrease in the TF oscillation amplitude taking place. This could be explained by the interaction mediated by a fluid-like contamination lay between two sliding solid boundaries (in this case these two boundaries are the tip and the sample). Owning to the correlation of TF and acoustic signals displayed on the curves, the tip-sample interaction exhibits dissipative and conservative properties. Furthermore, after passing through the region in which the TF-acoustic correlation is negative, the acoustic signal decreases with TF signal decreasing. The positive correlation between these signals could be attributed to the direct tip-sample contact, leading to a complete damping of TF oscillation and attenuation of acoustic signal. A description for an entire approach and retraction cycle is shown in Fig.4.3.14. At the beginning, the TF is freely oscillating in air (zone (1)). By bring the TF close to the sample, it interacts with a fluid-like contamination layer and a sound wave is generated due to (zone (2)). If approach process is continued, the tip-sample contact occurs, leading to the decrease in amplitude of both TF oscillation and acoustic signal (zone (3)). During 
the withdraw process, the tip is released by bring it away from the sample. However, retraction curves are different from approach curves due to the presence of contamination layer (zone (4)). After leaving the contamination layer, the $\mathrm{TF}$ is vibrating in air once again (zone (5)). However, the TF undergoes a longer distance from free oscillation to complete damping on hydrophilic surface (Fig. 4.3.13) than on hydrophobic surface (Fig. 4.3.12). This may be caused by the different physical nature of the boundaries at interplay with contamination layers on these two surfaces. Additionally, the hysteresis displayed in hydrophilic situation is more noticeable than in hydrophobic case.

Considering the chemical property of tip surface, PEOX-coated tip was used in the experiment as well. Fig. 4.3.15 shows the approach/retraction curves obtained when driving tip to oscillate with an ampliture of about $9 \mathrm{~nm}$. These results can support the analysis that we made above, since the main properties displayed on the curves remain the same. However, differences can be found by comparing the approach/retraction measurements shown in Fig. 4.3.14 and Fig. 4.3.15. The presence of differences could be caused by the replacement of tip . 


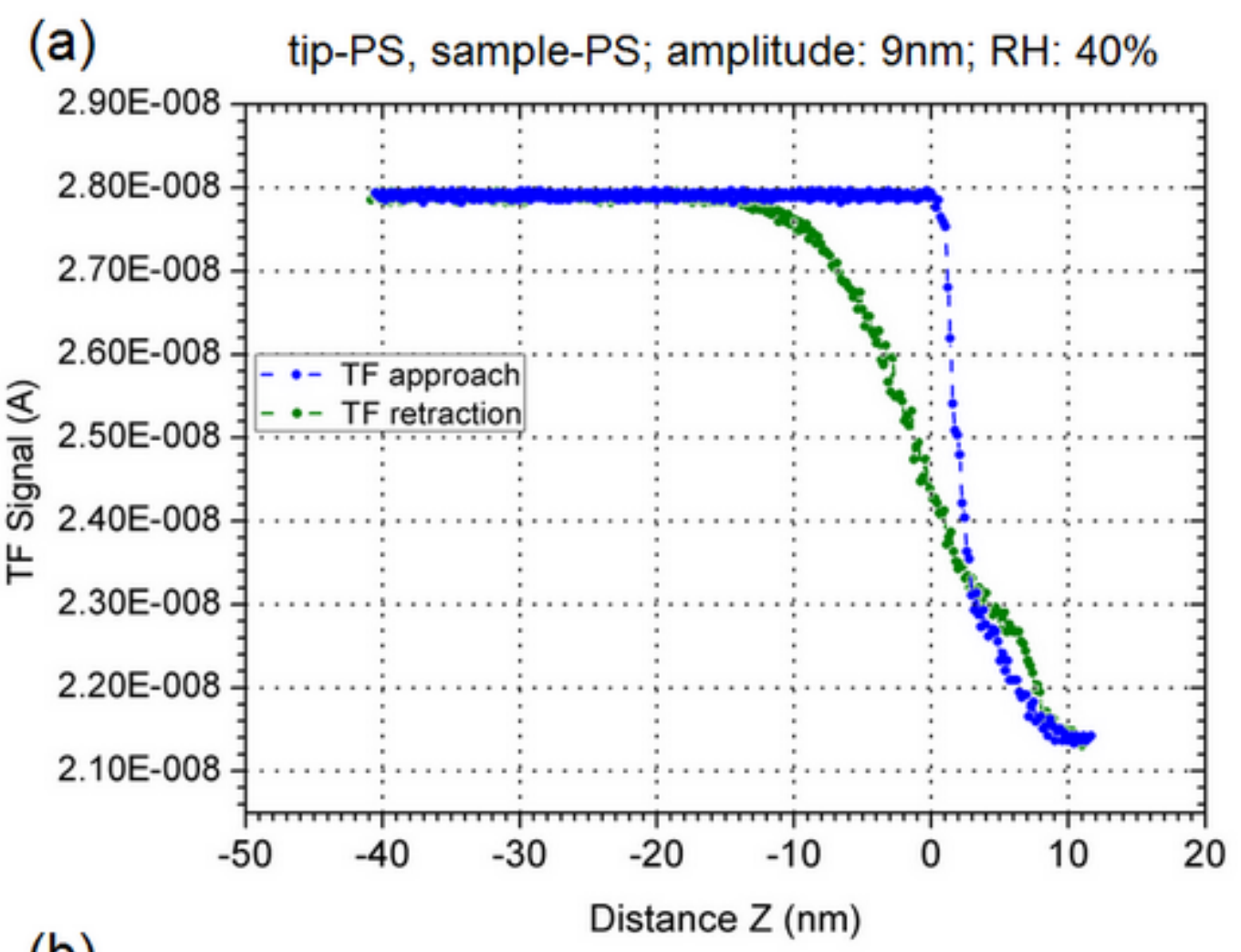

(b)

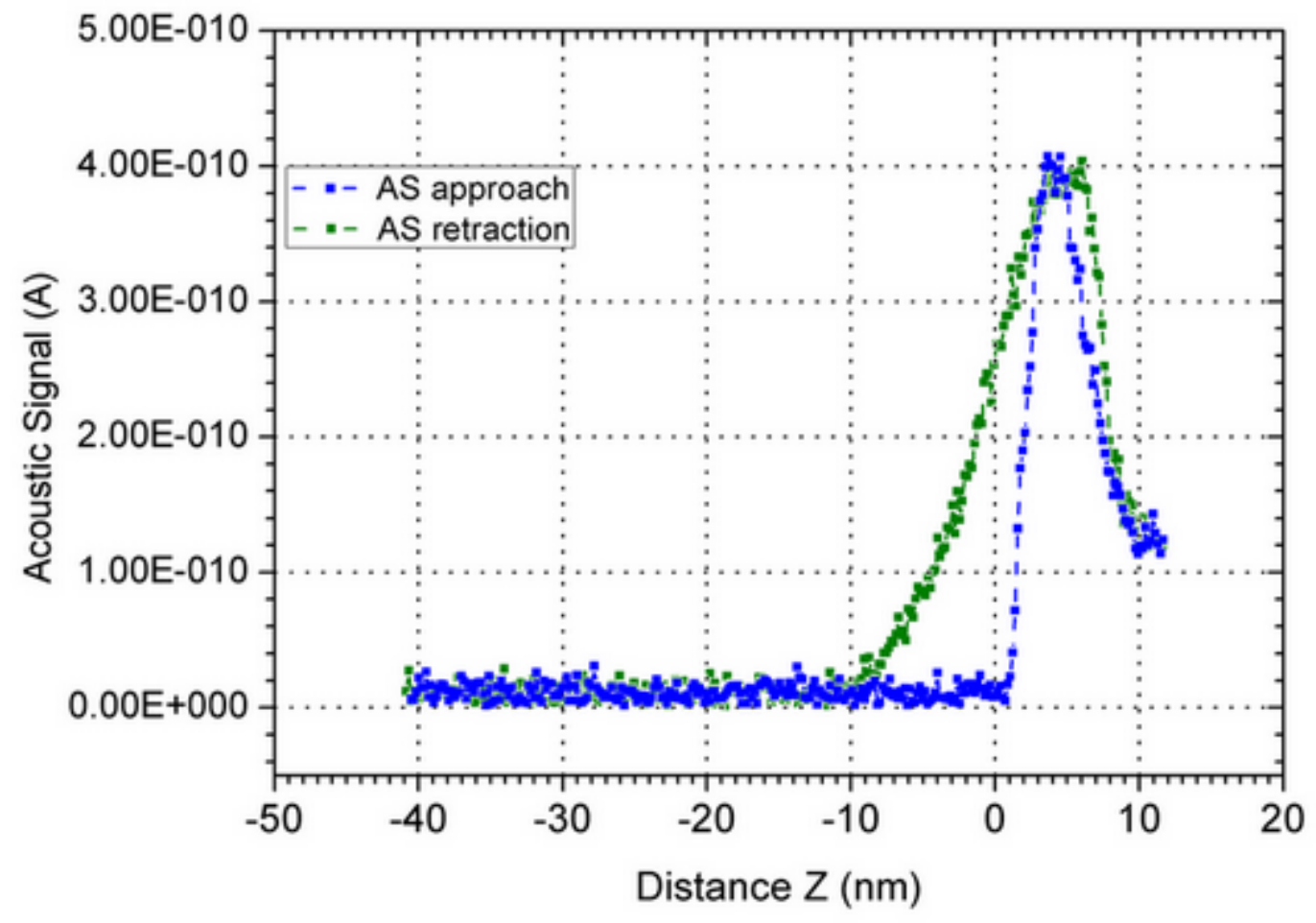

Fig. 4.3.12 Approach and retraction curves of (a) TF and (b) acoustic signals obtained when the PS coated gold tip (hydrophobic) approaching to and retracting from the surface covered by PS monolayer (hydrophobic). 


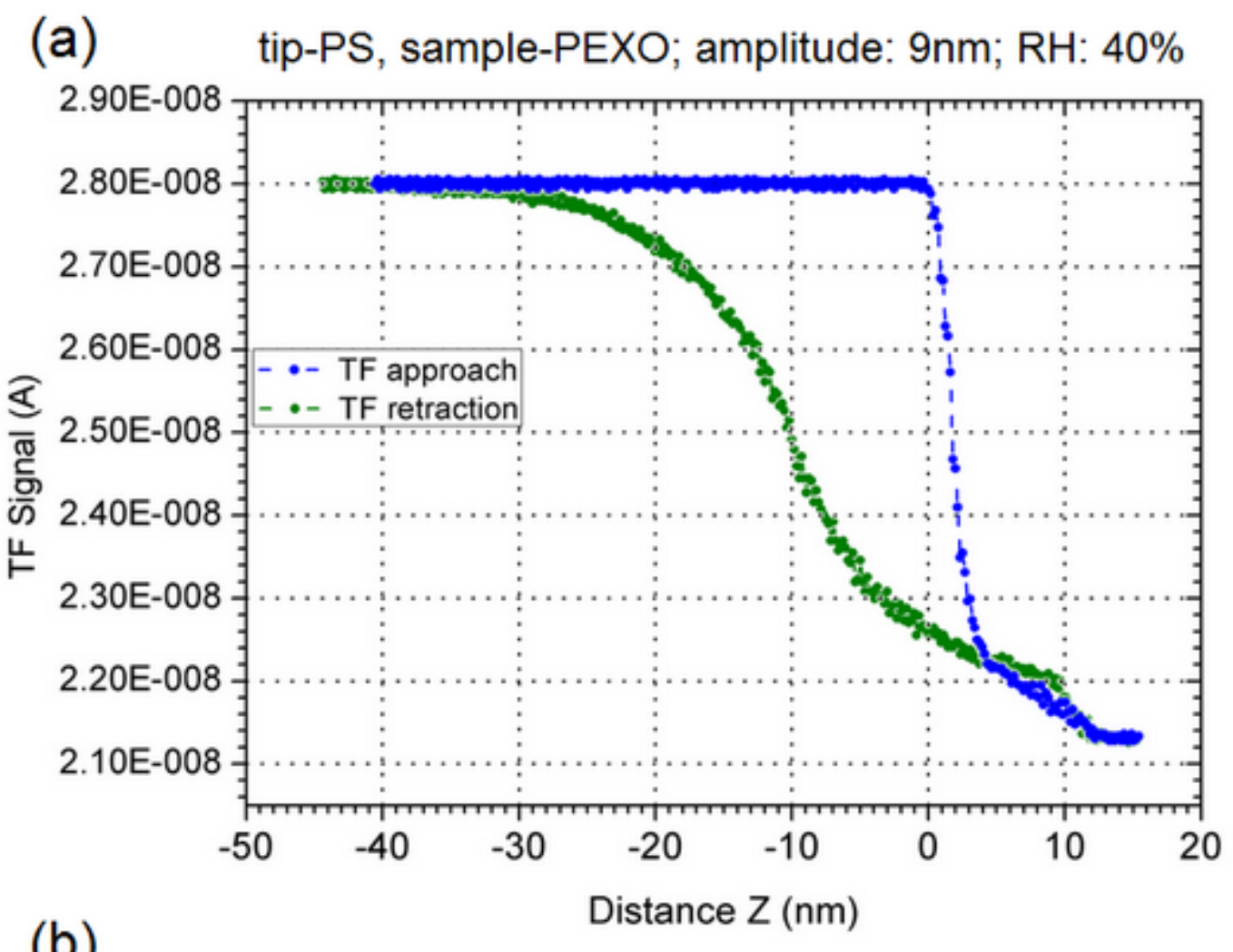

(b)

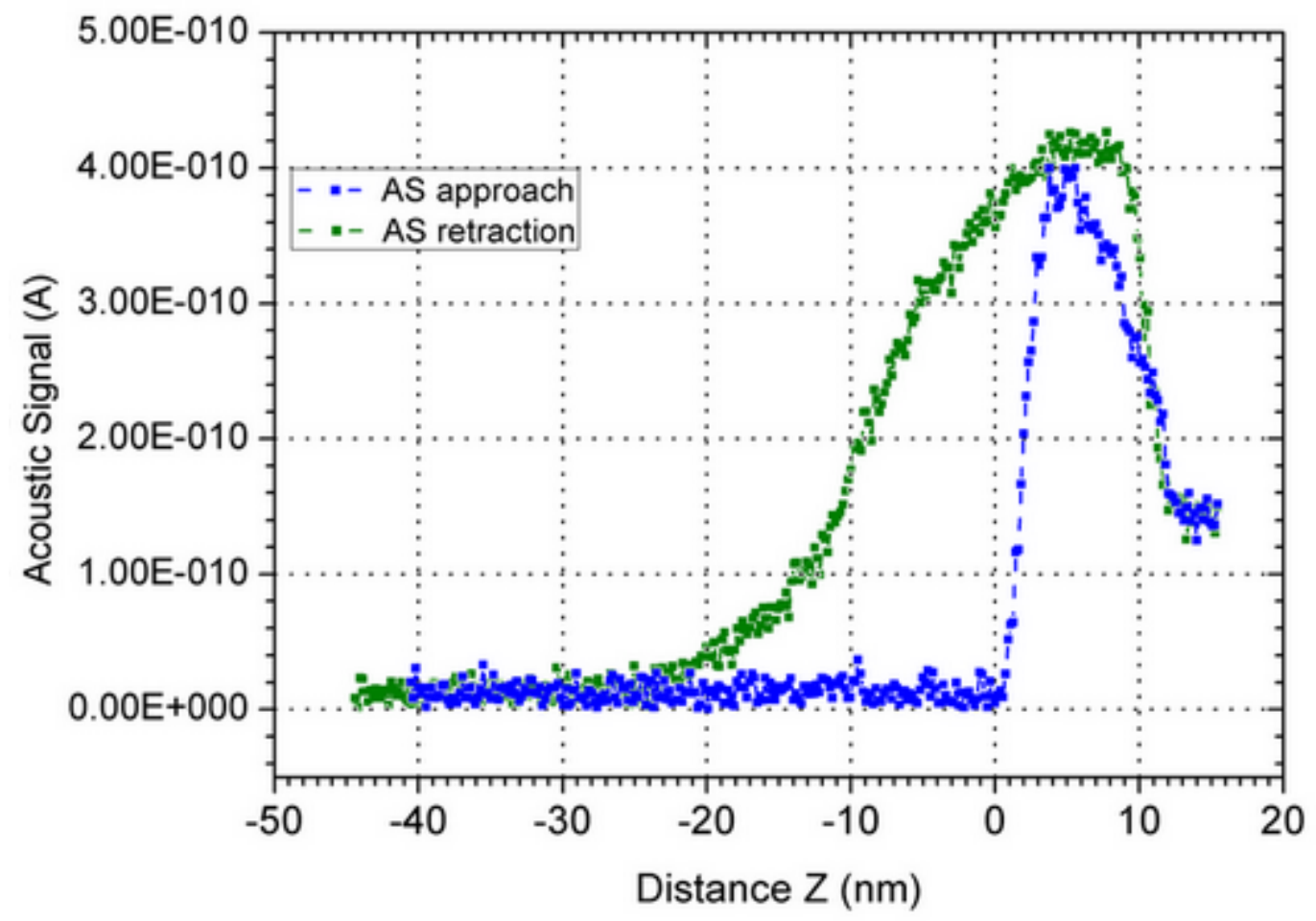

Fig. 4.3.13 Approach and retraction curves of (a) TF and (b) acoustic signals obtained when the PS coated gold tip (hydrophobic) approaching to and retracting from the surface covered by PEOX monolayer (hydrophilic). 

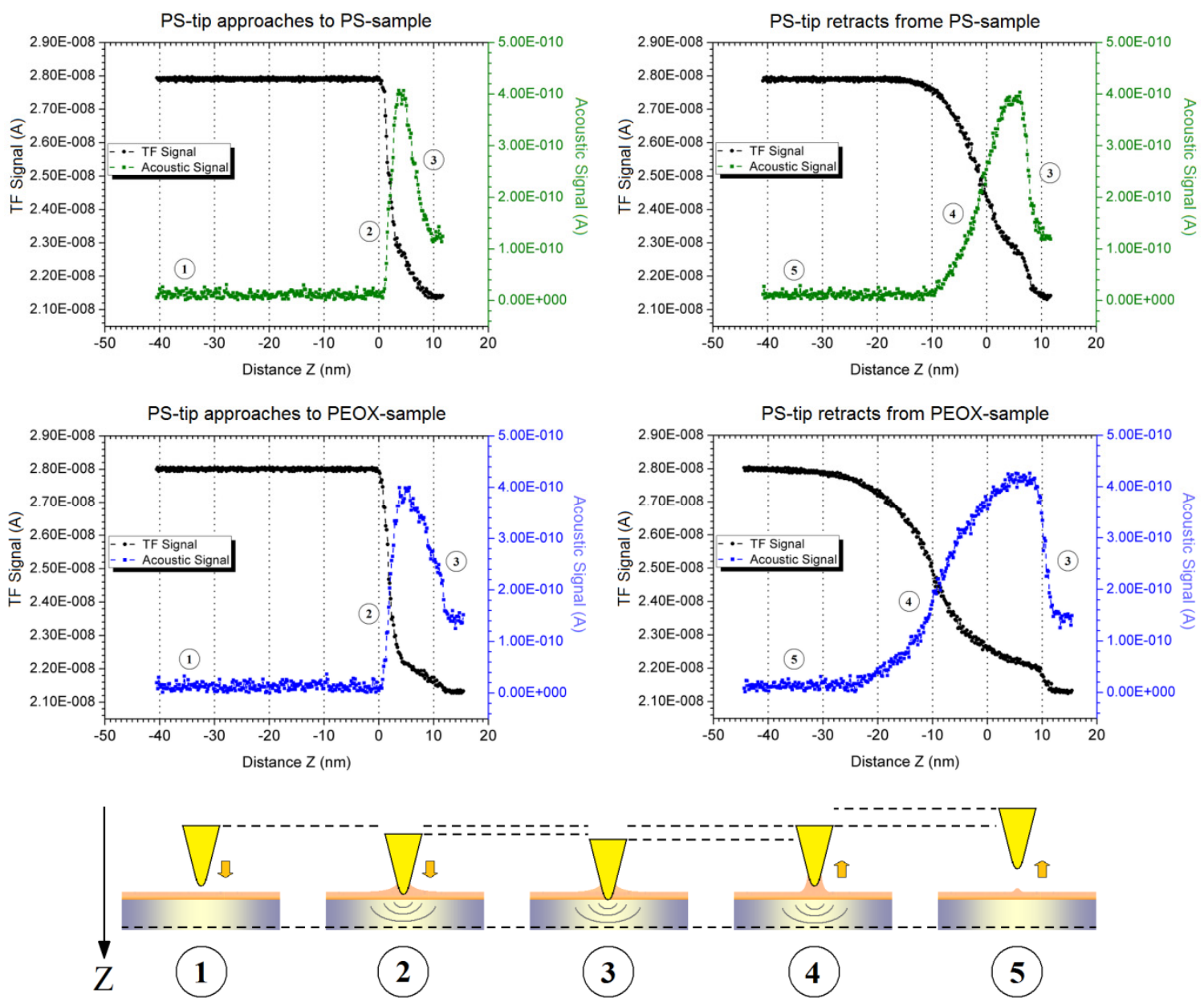

Fig. 4.3.14 The description for an entire approach and retraction cycle obtained using the gold tip coated with PS. 

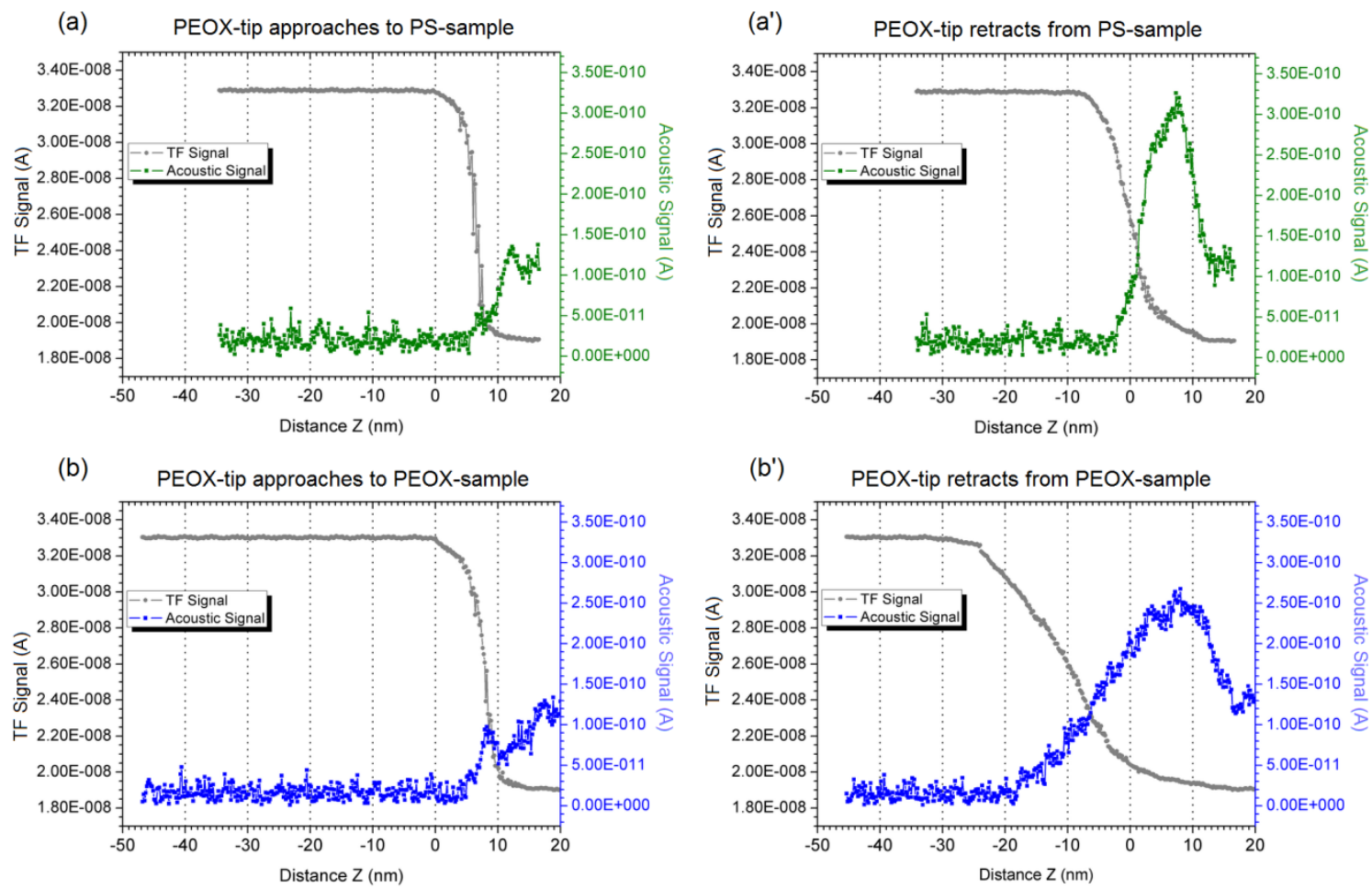

Fig. 4.3.15 Upper: TF (gray) and acoustic (blue) signals obtained when the PEOX-coated tip approaches (a) and retracts from (a') the hydrophilic surface. Lower: TF (gray) and acoustic (green) signals obtained by binging the same tip towards (b) and away from (b') the hydrophobic surface.

\subsection{Conclusions}

Our work allows a better understanding of the interaction between the tip and sample. Based on the approach/retraction measurements taken with the shearforce/acoustic microscope, more information with regard to the probe-sample interaction can be obtained from the TF signal as well as the acoustic signal. We found that when the tip approaches the sample surface, it comes in contact with a fluid-like contamination layer. Due to the presence of the contamination layer, capillary forces come into play but the effect can be different. Tips with larger surface area of apex (chubby tips) are influenced more by capillary forces than those having smaller surface area (sharp tips). After entering into the liquid layer, in most cases, a decrease of TF oscillation amplitude 
associated with an increase of acoustic signal is observed. It suggests that the probesample interaction is dissipative and conservative, mediated by the water layer existent between these two solid boundaries. With a further reduction of the probe-sample separation, the tip gets into contact with the sample, and the oscillation amplitude is completely damped, leading to a decrease of acoustic signal. The effects of environmental humidity and chemical property of sample surface are discussed as well. These studies allow testing the influence of contamination layer on the probe-sample interaction. Indeed, either changing the humidity or the water affinity of sample surface impacts the obtained approach/retraction curves. It indicates that the fluid-like layer plays a critical role in the probe-sample interaction. If the TF signal is said to be concentrated on the gas/liquid interface (the interface of air and contamination layer), the acoustic detection is more sensitive on the liquid/solid interface (the interface of contamination layer and sample surface). Acoustic measurements can provide more information than the TF signal when the tip is brought in proximity of the sample surface. 


\section{CHAPTER 5. CONTACT CURRENT: THE INDICATOR OF SOLID-SOLID TIP- SAMPLE MECHANICAL CONTACT}

\subsection{Motivations}

The studies in previous chapter demonstrate the importance of the contamination layer in shear force damping when performing experiments in the ambient environment. By placing the acoustic sensor under the sample, a wealth of information about the probeto-sample interaction can be retrieved from data of the $\mathrm{TF}$ and acoustic sensor recorded simultaneously. However, since mesoscopic water films display characteristic different than the bulk, depending on the surface organization and on the probe behavior itself, it becomes difficult to accurately locate the position of probe only based on the measurements of TF and acoustic signals. In this chapter, a simple strategy for characterizing the tip-sample contact comes out when applying a bias voltage between the metallic tip and the conducting sample, thus the contact current, indicating the tip-

sample mechanical contact, can be monitored. ${ }^{[42,43]}$ Furthermore, the relationship among the contact current, TF and acoustic signals will be investigated for better understanding shear force damping and other surface phenomena.

\subsection{Experimental Section}

An experimental settings similar to the one for detecting the hydrophobic/hydrophilic interactions, as described in the previous chapter, was used in this case. A schematic of the settings is shown in Fig. 5.2. The setup consists of three major components for signal detection: (1) the "shear-force" measuring system composed of a quartz tuning fork and a lock-in amplifier (Lock-in \#1, SR850, Stanford Research 
System); (2) the acoustic signal measuring system, which consists of the acousitc sensor placed underneath the sample and a lock-in amplifier (Lock-in \#2, SR850, Stanford Research System); (3) the "contact current" measuring system, including an external signal generator, a current preamplifier, and a lock-in amplifier (Lock-in \#3, SR844, Stanford Research System).

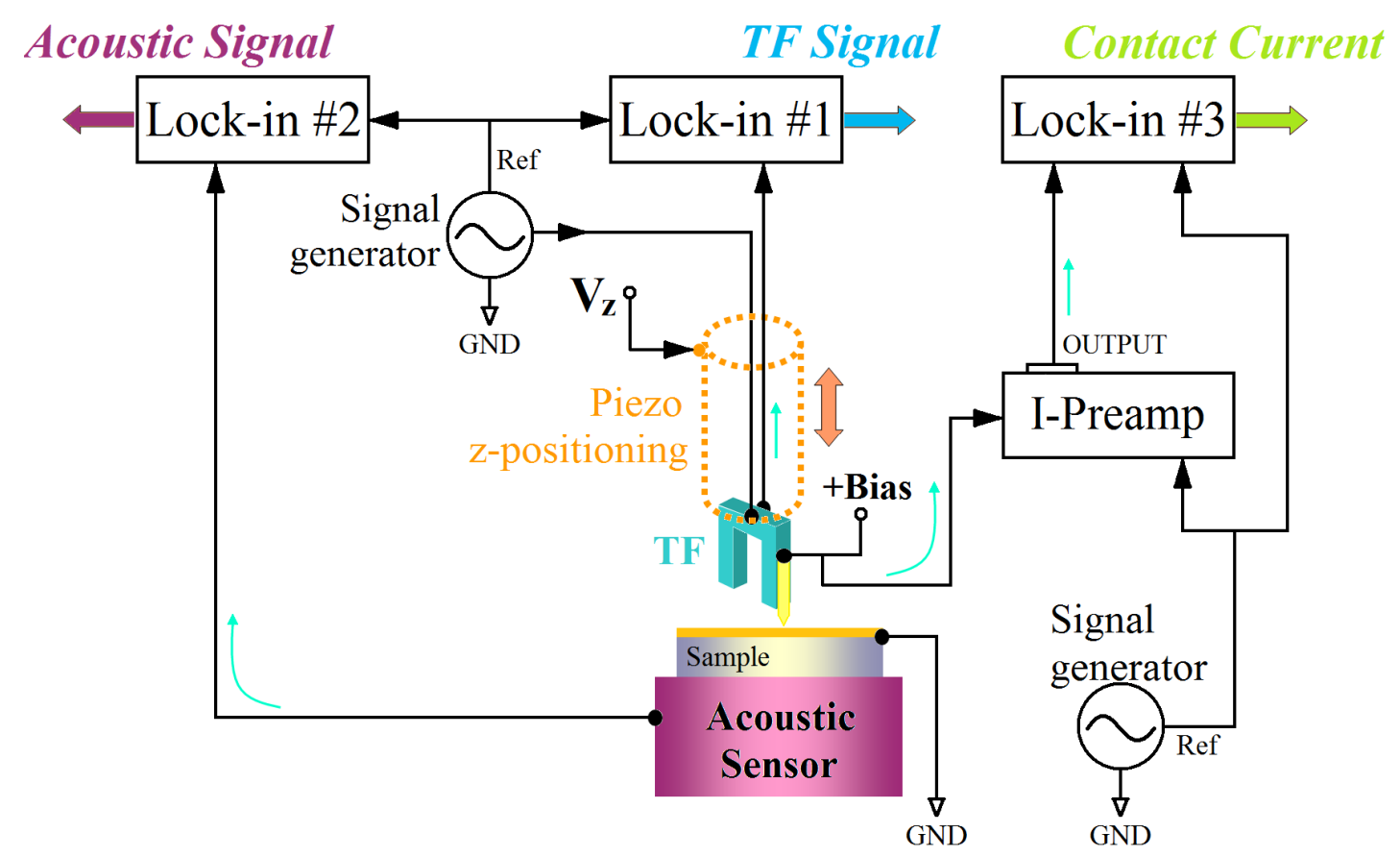

Fig. 5.2 Schematic of the settings used for detecting the contact current, along with the tuning fork (TF) and the acoustic signals.

A long gold tip (about $8 \mathrm{~mm}$ long) prepared by electrochemical etching was manually mounted to one of tines of the TF acting as the probe. The TF was implemented as the shear-force sensor when it was excited at or near its resonance frequency by a sine wave from the internal reference source of the lock-in amplifier. Attaching the tip to the $\mathrm{TF}$ led to an increase in the total mass, lowering the resonance frequency. ${ }^{[44]}$ Additionally the quality factor Q (defined as the ratio of the resonance frequency to its width measured at full width at half maximun) decreased upon tip attachment due to the increased energy 
dissipation of both glue and tip. The measurement of mechanical oscillation amplitude of the tip-mounted TF used the piezoelectric effect inherent to quartz crystals providing an electric signal (TF signal) proportional to the applied driving voltage. The conducting sample was obtained by sputter coating a thin layer of gold ( $30 \mathrm{~nm}$ thick) onto the silicon substrate. An adjustable $\mathrm{DC}$ bias voltage ranging from $-5 \mathrm{~V}$ to $+5 \mathrm{~V}$ was applied between the metallic tip and the conducting surface by a low-noise current preamplifier (SR570, Stanford Research System). This arrangement allowed simultaneous detection of the current that indicated when the probe made contact with the solid surface. An acoustic sensor (SE40-Q, Dunegan Engineering Consultants, Inc.) placed under the substrate was used to detect the acoustic signal created by the tip that oscillated parallel to the sample surface.

To achieve the approach/retraction operation, the TF was attached to a piezo stage (Nano-OP65, Mad City Labs, Inc.) which can provide $65 \mu \mathrm{m}$ range of motion in vertical direction (z-direction) with nanometer accuracy. The hysteresis arisen from the piezo stage can be completely eliminated. In a typical experimental cycle, the probe was set to vibrate with an amplitude of a few nanometers by driving the TF at its resonant frequency, then it moved slowly towards and away from the sample surface. As shown in Fig. 5.2, during the approach/retraction process, the responses of TF and acoustic sensor were synchronously recorded by the lock-in amplifier $\# 1$ and $\# 2$ as the TF signal and the acoustic signal, respectively. By applying a bias voltage of $50 \mathrm{mV}$, the appearance of current can be monitored by the current preamplifier that connected to the lock-in amplifier \#3 when the metallic tip got into contact with the conducting sample. 


\subsection{Results and Discussion}

When the tip's apex was positioned far away enough from the surface (i.e., $1 \mathrm{~mm}$ ), the TF was freely oscillating in air. Fig. 5.3.1 shows a typical TF spetrum obtained by performing the frequency sweep. By using a driving voltage of $20 \mathrm{mV}$, the TF spectrum displayed a resonance peak at $f_{\text {res }}=30524 \mathrm{~Hz}$, and the quality factor of $Q=f_{\text {res }} /(\Delta f)_{1 / 2}: 160$. The peak current of TF at the resonance frequency was selected at $I_{\max } \approx 2.080 \times 10^{-8} \mathrm{~A}_{r m s}$ (root-mean-square amplitude of the alternating current), corresponding to the maximum amplitude of TF oscillation. The current corresponding to the minimum amplitude of TF oscillation was chosen as $I_{\min } \approx 1.940 \times 10^{-8} \mathrm{~A}_{r m s}$. The amplitude of free oscillation was estimated to be about $8 \mathrm{~nm}$.

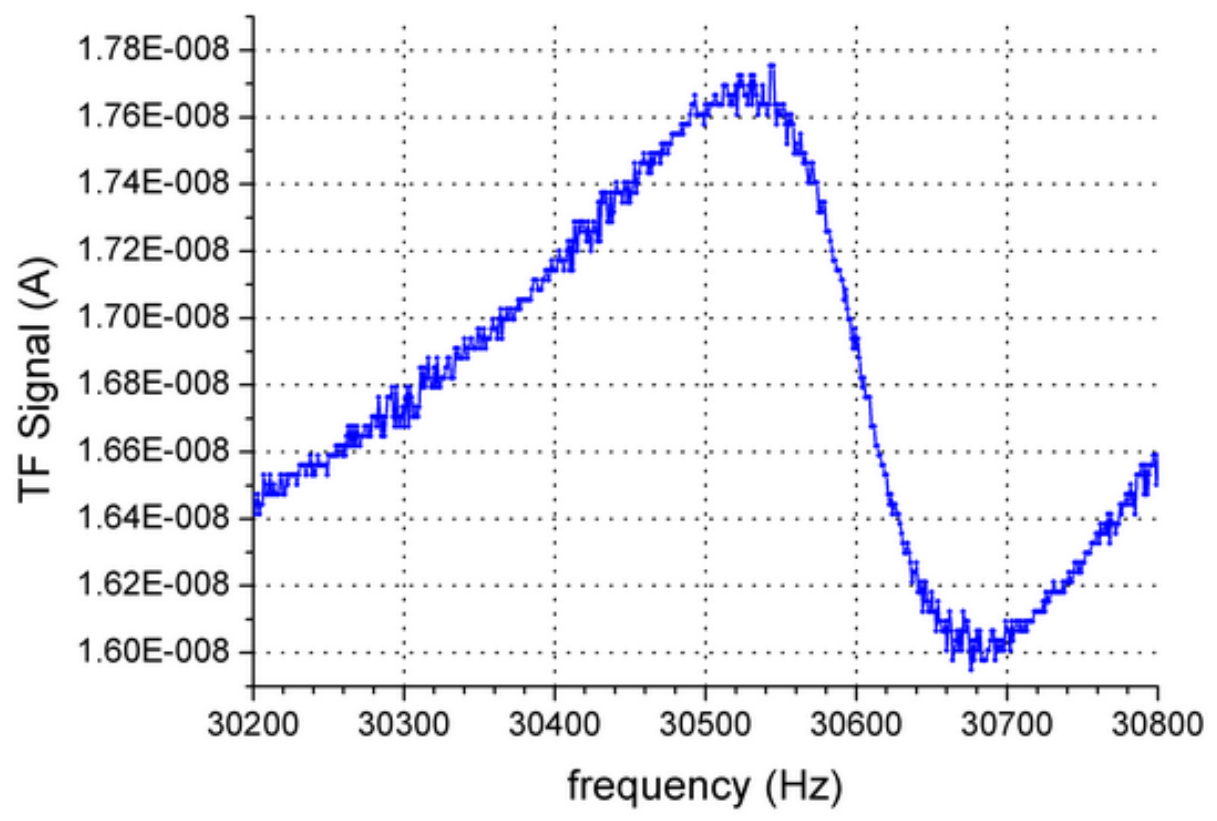

Fig. 5.3.1 The spectrum of the tuning fork oscillating in air obtained by performing the frequency sweep from $30200 \mathrm{~Hz}$ to $30800 \mathrm{~Hz}$.

Approach and retraction curves were obtained to show how the TF and acoustic signals varied while the tip approached and retracted from the surface at ambient air. The 
intensities of TF and acoustic signals are plotted as a function of the tip-sample distance $\mathrm{Z}$ in Fig. 5.3.2. As the tip gets closer to the sample, both dissipative and reactive forces are experienced by the tip, resulting in a decrease in the amplitude of the TF. The position where the TF signal starts to drop is arbitrarily set to be the zero position. As for Fig. 5.3.2 a significant difference between approach and retraction curves is observed. This is probably due to the tip entering and leaving a fluid-like layer formed by attracting moisture from the surrounding. Furthermore, the acoustic signal begins to increase once the TF signal starts to decrease during the approach process. A satisfying explanation could be that the acoustic signal arises from the dissipative and conservative interaction that takes place where the tip is contact with the fluid-like layer or/and the solid surface. 

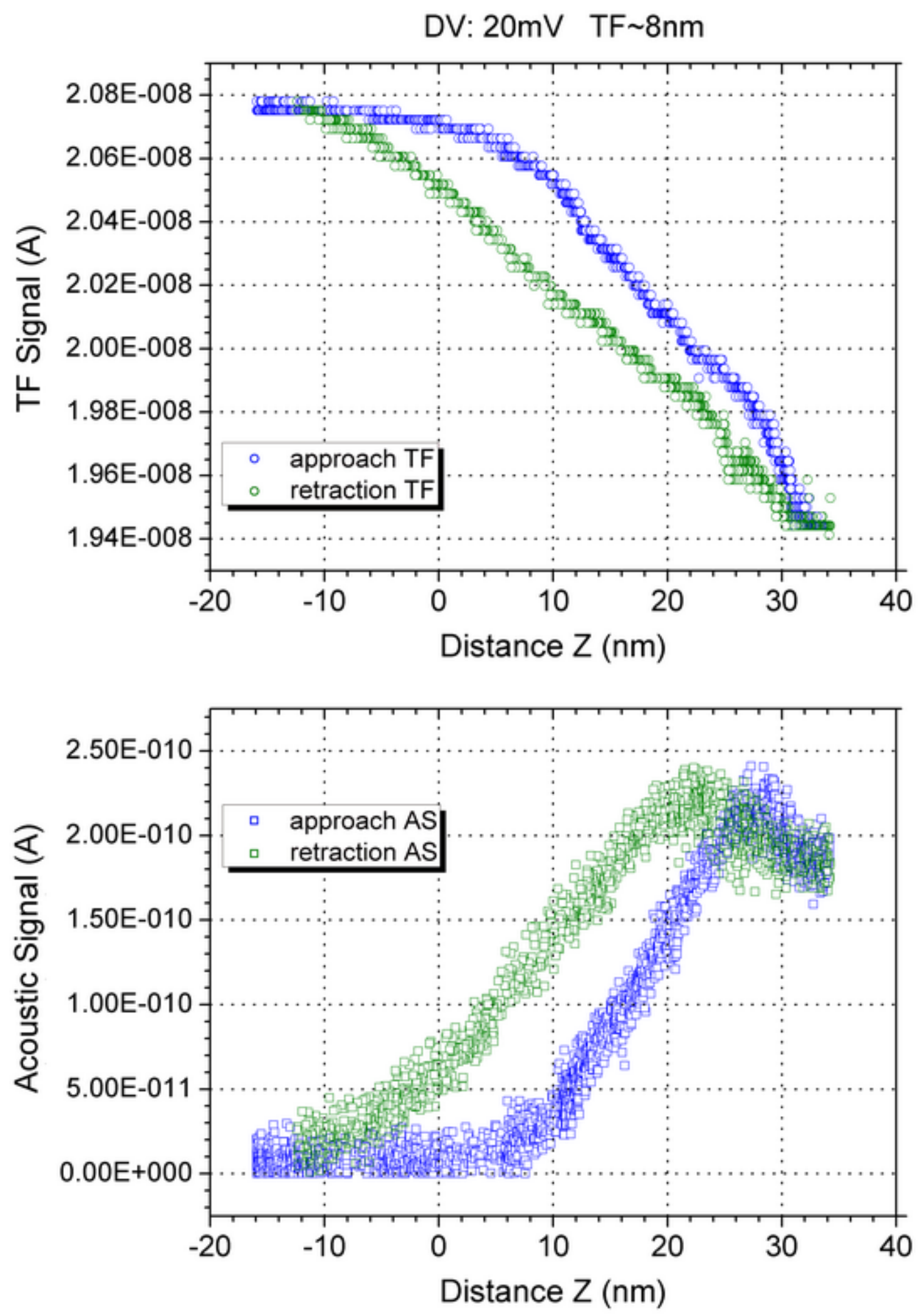

Fig. 5.3.2 The approach and retraction curves of TF and acoustic signals simultaneously recorder by the lock-in amplifiers. 
Fig. 5.3.3 shows simultaneously measured TF damping (blue), the acoustic response (green) and "contact" current (red) dependences on the tip-sample distance. The data recorded while the tip slowly approaching the sample can be divided into three regions labeled in Fig.5.3.3. In region (1), the tip freely vibrates at its excitation amplitude. In region (2), the TF and acoustic signals vary without the presence of tip-sample contact current. It implies that the shear-force damping cannot be completely attributed to a hard mechanical contact. Furthermore, the acoustic signal increases as the oscillation of TF is damped during the approach. The correlation between these two signals is "negative". Owning to the existence of the contamination layer on the sample surface, viscous forces and other forces (i.e., electrostatic forces) are acting on the tip when oscillating in the thin layer, leading to the shear-force damping and generation of sound waves, which subsequently propagate through the sample. In region (3), the appearance of contact current is observed, indicating that the tip has probably gotten into contact with the conducting surface. Moreover, the correlation between the TF and acoustic signals becomes "positive", which means that the acoustic signal decreases with decreasing the TF signal. This could be attributed to friction resulted from direct contact of the tip on the sample. When contact occurs, the presence of nonzero force acting at the tip apex leads to friction. Additionally, a steeper slope of the TF curve is observed in this region, this could be explained by the occurrence of mechanical contact between the tip and the solid surface. Finally, if the approach is continued, the tip sticks onto the surface so that its vibration amplitude falls to a minimum. 


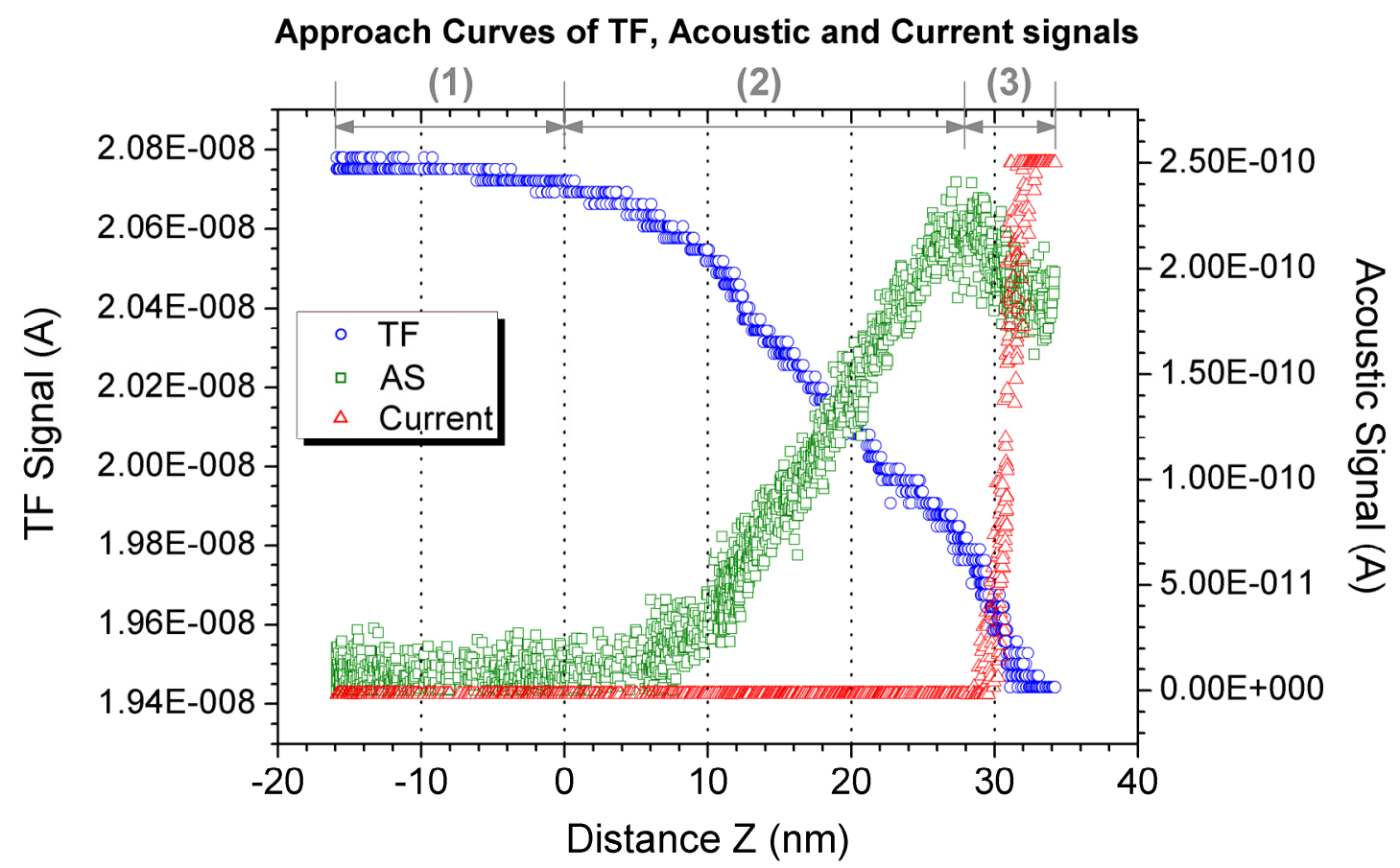

Fig. 5.3.3 Simultaneously measured TF damping, the acoustic response and "contact" current dependences on the tip-sample distance during the approach.

\section{Retraction Curves of TF, Acoustic and Current signals}

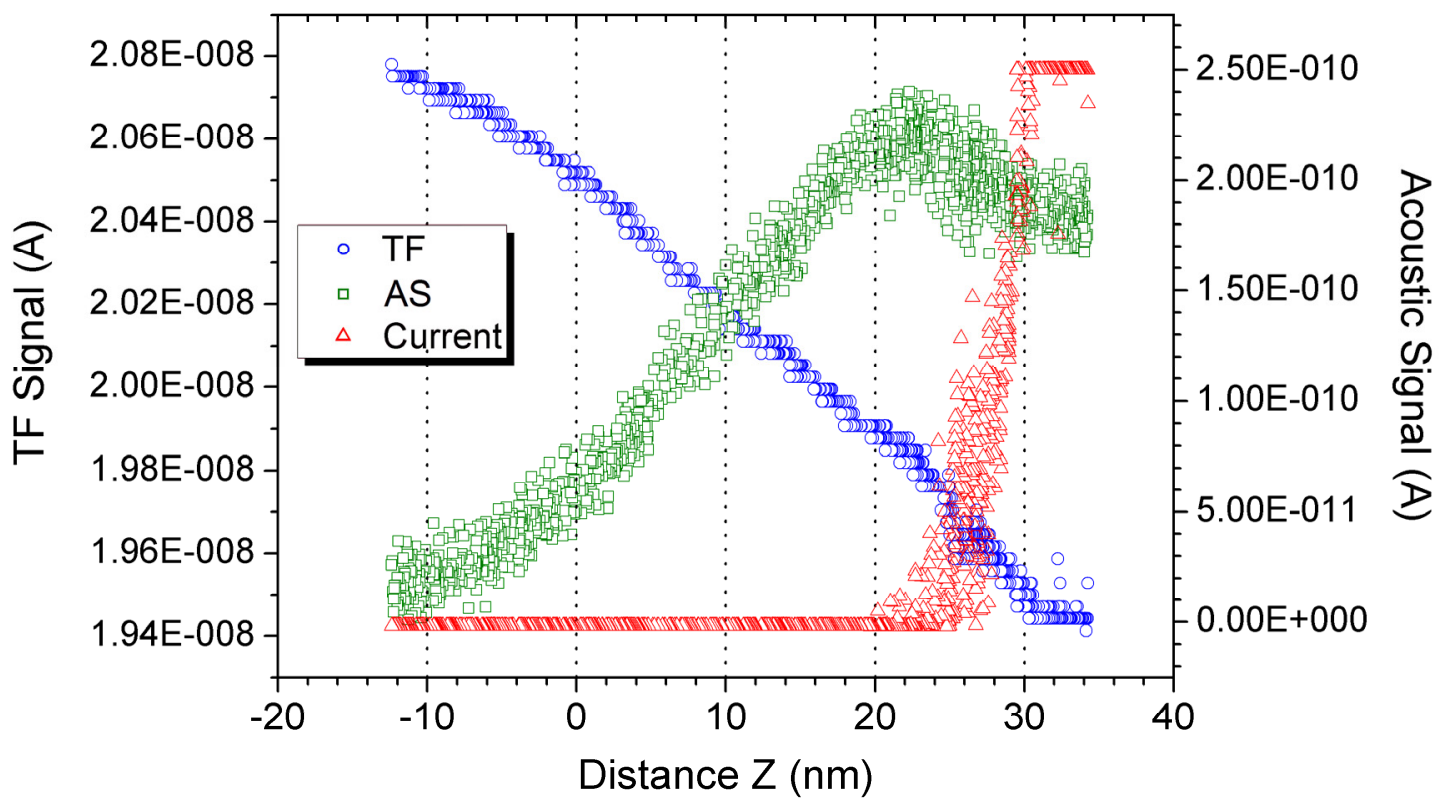

Fig. 5.3.4 Simultaneously measured TF damping, the acoustic response and "contact" current dependences on the tip-sample distance during retraction. 
The respective retraction curves of the TF and acoustic signals, along with the contact current are shown in Fig. 5.3.4. A similar analysis can be applied by separating these curves into three zones, and details will not be repeated here. In both approach and retraction cases, it is difficult to avoid saturation of the current preamplifier when using it to monitor the contact current, but it still gives a qualitative impression on the current behavior that indicates the tip-sample contact.

To support our claims the experiment was repeated with the same tip and sample, but by changing the location of tip to perform the approach/retraction operation. And the resulting curves shown in Fig. 5.3.5 and 5.3.6. Although these plots slightly differ from those obtained previously (see Fig. 5.3.3 and Fig. 5.3.4) because of the complexity of the surface, the main properties of the curves are the same, as we expected.

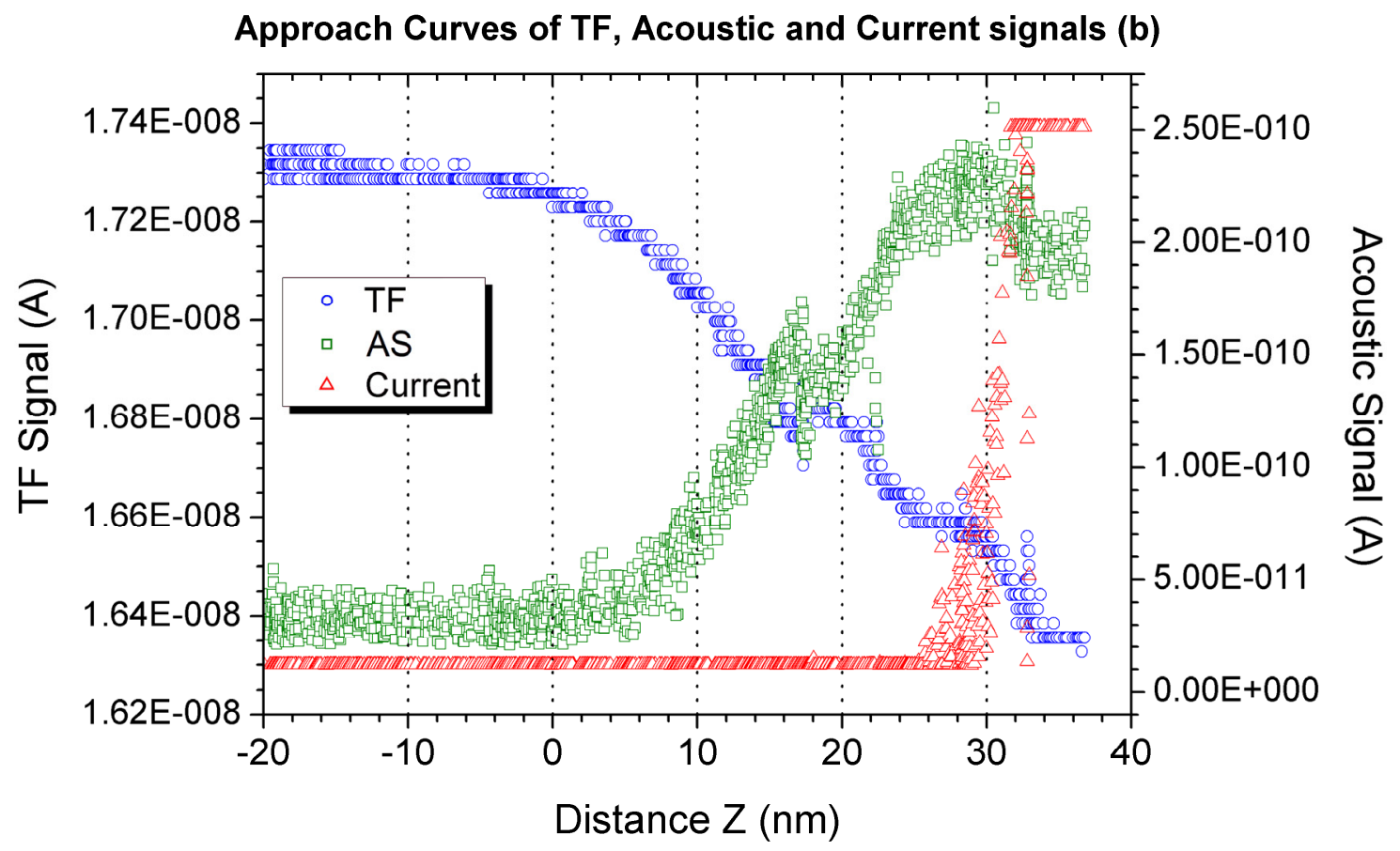

Fig. 5.3.5 Approach curves of TF, acoustic and current signals obtained by repeating the approach/retraction experiment at a different location. 


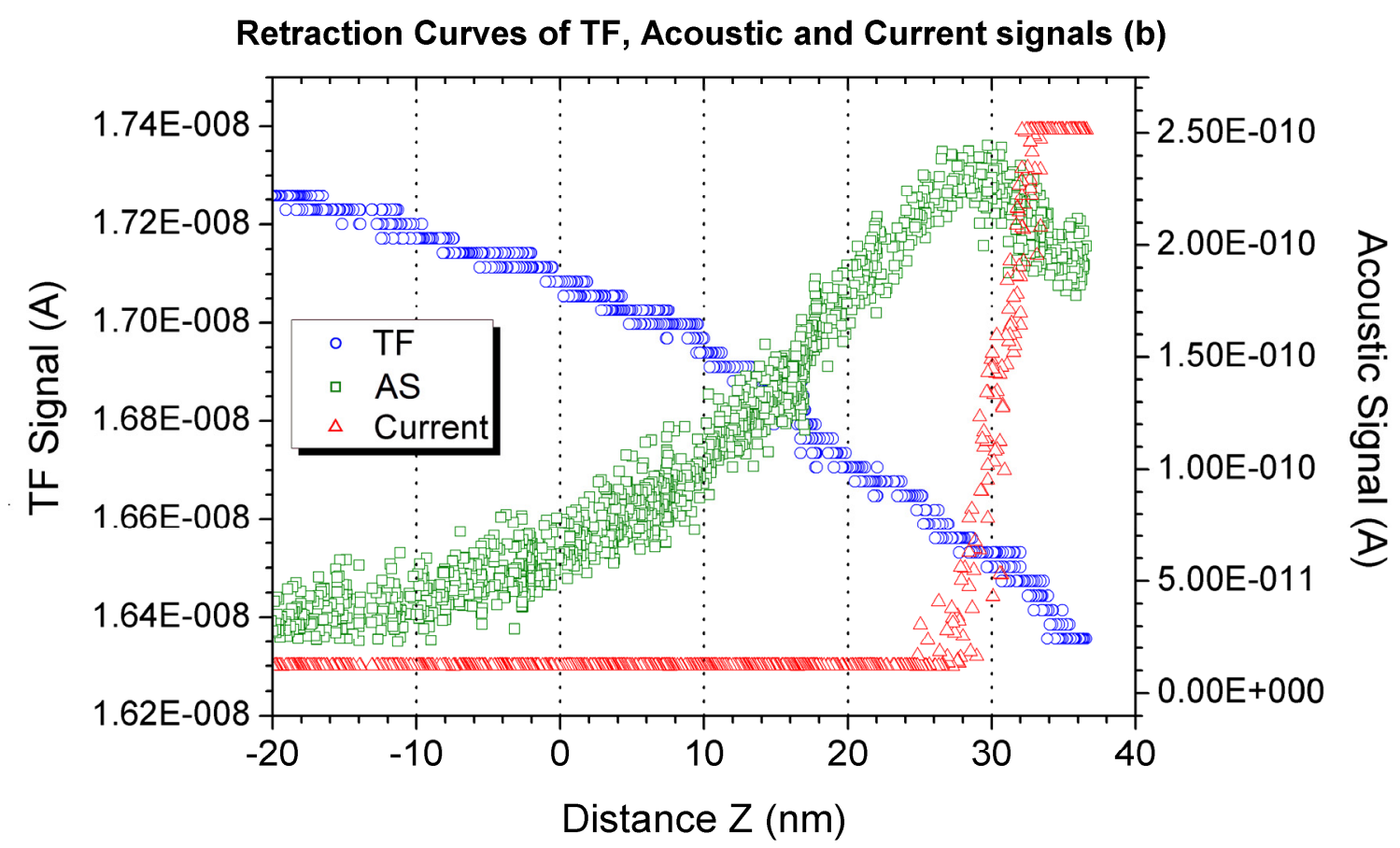

Fig. 5.3.6 Retraction curves of TF, acoustic and current signals obtained by repeating the approach/retraction experiment at a different location.

\subsection{Conclusions}

The experimental results show that the shear-force signal begins to damp before the mechanical contact occurs. The damping effect could be attributed to the interaction forces such as viscous force and capillary force acting on the tip when the tip enters a contamination layer on top of the solid surface. One evidence that supports the existence of the adsorbed layer is the hysteresis displayed in the approach/retraction curves. After eliminating the hysteresis of the piezo stage, the mismatch of the approach and retraction curves probably results from the tip entering and leaving a liquid layer. Furthermore, due to the presence of the fluid-like layer, sound waves could be generated while tip vibrating in this medium, and subsequently received by the acoustic sensor placed under the sample. Additionally, applying a bias voltage between the metallic tip and the conducting 
sample could allows a current signal being detected when the tip gets into contact with the solid surface. Although it is difficult to avoid the saturation of the current signal, this design gives a qualitative impression on the current behavior that indicates the tip-sample contact. 


\section{CHAPTER 6. ELECTRIC FIELD-ASSISTED DIP-PEN NANOLITHOGRAPHY ON P4VP THIN FILMS}

\subsection{Introduction}

Nanolithography comprises a set of technologies capable of etching, writing, or patterning materials with nanometer scale precision, which nowadays has widespread applications in electronic engineering, physics, chemistry and biology. Dip-pen nanolithography (DPN), ${ }^{[45]}$ as a scanning-probe-based lithography, is used to directly deliver atoms or molecules (initially attached by adhesion to an atomic force microscope (AFM) tip) onto substrates of interest in a controlled fashion. A diverse range of molecular species such as small organic molecules, ${ }^{[45]}$ biological macromolecules, ${ }^{[46]}$ conducting polymers, ${ }^{[47]}$ metal ions and nanopaticles, ${ }^{[48,49]}$ have been deposited via DPN process onto surfaces of proper affinities. In addition, the patterning substrates have been expanded to metals, ${ }^{[50]}$ insulators, ${ }^{[50]}$ semiconducting materials, ${ }^{[51]}$ and functional monolayers adsorbed on substrates. ${ }^{[52]}$ Applications of DPN have been envisioned in nanoelectronics, ${ }^{[53]}$ microfluidics, ${ }^{[54]}$ molecular electronics (organic circuits), ${ }^{[55]}$ and nanosensors for biological and chemical species, ${ }^{[56]}$ etc. In most cases, the transport of "ink" molecules from the tip to the substrate occurs in a liquid bridge naturally formed between them through capillary condensation. Hence, this technique has also been used to experimentally study the mechanism of molecular diffusion through nanoscale junctions and the in situ growth of self-assembled monolayers under ambient conditions. ${ }^{[57,58]}$ Furthermore, due to its direct-write capability, DPN is suitable for investigating responsive films at the mesoscopic scale. 
Responsive films made of self-assembled monolayers (SAMs) or polymer thin films are valuable in the development of electronic, analytical and biomedical devices. These films exhibit different properties in response to external signals, such as the changes in temperature, $\mathrm{pH}$, light, and ionic strength. Poly(4-vinylpyridine) (P4VP), a weak polyelectrolyte, can be prepared as polymer thin films tethered to solid supports simply by spin coating and subsequent UV irradiation. ${ }^{[40]}$ The immobilization results from the UV induced cross-linking of polymer leading to the attachment of polymer film to the substrate. Moreover, P4VP film shows a pH-dependent response that causes the structural changes of the polymer and controls surface wettability. ${ }^{[59]}$ The reversible transformation of $\mathrm{P} 4 \mathrm{VP}$ originates from the protonation of pyridine units of the polymer backbone at the acidic $\mathrm{pH}$, thus providing the basis for applications of $\mathrm{P} 4 \mathrm{VP}$ films as responsive coatings. Recently, P4VP polymer-brush-functionalized electrodes have been designed for future "smart" bioelectronic devices ranging from biosensors to biofuel cells. ${ }^{[60,61]}$ Additionally, $\mathrm{P} 4 \mathrm{VP}$ is an interesting material because of its tunable photoluminescence (PL) properties. Vaganova et al. demonstrated that P4VP with protonated polymeric pyridine groups displayed photoactive emission properties, which could be tuned to glow blue, red or green, depending on the pumping wavelength. ${ }^{[62]}$

Herein we report the fabrication of patterns on P4VP thin films based on DPN technique by exploiting the swelling of the polymer. Differing from the feature formation via a direct deposition of ink molecules onto substrates, the underlying principle in our approach is to locally trigger the protonation of $\mathrm{P} 4 \mathrm{VP}$ for creating swollen patterns by delivering acidic ions into the polymer. Moreover, a reliable strategy for well controlling the patterning process results when applying an electric field between the AFM tip and 
the sample. We demonstrate the capability of the electric field-assisted DPN technique for reproducibly and reliably fabricating nanostructures. Our work includes a systematic study of pattern formation under different patterning parameters (i.e., externally applied bias and tip-sample contact force), and more importantly, provides evidence on the reversible characteristic of this process.

\subsection{Experimental Section}

Polymer Thin Film Preparation: P-type silicon (100) wafers were cleaned by immersion in piranha solution $\left(35 \% \mathrm{H}_{2} \mathrm{O}_{2} / \mathrm{H}_{2} \mathrm{SO}_{4}=3: 7(\mathrm{v} / \mathrm{v})\right)$ at $80-90^{\circ} \mathrm{C}$ for $60 \mathrm{~min}$ and were subsequently washed with boiling water for $90 \mathrm{~min}$, then dried under $\mathrm{N}_{2}$. The fleshly cleaned wafers were then dipped into HF aqueous solution to remove the native oxide layer from the silicon surface, rinsed thoroughly with Milli-Q-water, and dried with $\mathrm{N}_{2}$. A $10 \mathrm{mg} / \mathrm{ml}$ solution of P4VP (molecular weight ca. 160000) in n-butanol was spin coated onto silicon substrates at $2000 \mathrm{rpm}$ for $60 \mathrm{~s}$. The polymer-coated substrates were irradiated with a $450 \mathrm{~W}$ medium-pressure mercury lamp for $15 \mathrm{~min}$ (the irradiation time included approximate 2 min warm up for the UV lamp to reach its maximal intensity). The unbound polymers were removed by soaking the irradiated substrates in n-butanol for $12 \mathrm{~h}$. The remaining films were dried with $\mathrm{N}_{2}$, and the thickness was measured on a Gaertner Model L116A ellipsometer with a $632.8 \mathrm{~nm} \mathrm{He} / \mathrm{Ne}$ laser at an incident angle of $70^{\circ}$. The refractive index used in the model to determine the film thickness was 1.581 for P4VP. An average thickness of $40 \mathrm{~nm}$ was obtained after taking measurements at three different locations. 
Force Calibration: Silicon-based AFM tips of high spring constant coated with 15$\mathrm{nm}$ thick wear-resistant platinum (NSC15/Pt, $k \approx 40 \mathrm{~N} / \mathrm{m}$, MikroMasch) were used for surface patterning and imaging. The normal spring constant of each cantilever was calibrated using the Sader method, ${ }^{[63]}$ which states $k=0.1906 \rho_{f} b^{2} L Q_{f} \omega_{f}^{2} \Gamma_{i}^{f}\left(\omega_{f}\right)$, where $L$ and $b$ are the length and width of the cantilever, respectively, $\rho_{f}$ is the density of air $\left(1.18 \mathrm{~kg} / \mathrm{m}^{3}\right.$ at $\left.25^{\circ} \mathrm{C}\right), \omega_{f}$ and $Q_{f}$ are the resonant frequency and quality factor of the fundamental resonance peak in air, respectively, and $\Gamma_{i}^{f}$ is the imaginary part of the hydrodynamic function.

Feature Patterning and Characterization: All the experiments were performed with a commercial AFM (XE-70, Park Systems) in ambient environment $\left(23-24^{\circ} \mathrm{C}\right.$ and $45-$ $55 \%$ relative humidity). Prior to the patterning process, the AFM tip was inked by dipping into a phosphate buffer solution for $1 \mathrm{~min}$ and blown dry with $\mathrm{N}_{2}$. Buffer solutions $(100 \mathrm{mM})$ of $\mathrm{pH} 4.0$ and $\mathrm{pH} 8.3$ were prepared by dissolving $\mathrm{NaH}_{2} \mathrm{PO}_{4}$ and $\mathrm{Na}_{2} \mathrm{HPO}_{4}$ in appropriate proportion in distilled water. The sample was grounded while a bias was applied to the tip. The voltages were quoted by referencing the polarity of the tip. The bias voltage was held constant while the tip transcribed the preprogrammed patterns controlled by the AFM in contact mode. To visualize the resulting patterns, topographic images were subsequently acquired by operating the AFM in non-contact mode with the same tip used for patterning.

\subsection{Results and Discussions}

As outlined in Fig. 6.3.1, a series of systematic experiments were carried out to study the mechanism of pattern formation as well as the effects of patterning parameters 
(e.g., externally applied bias and tip-sample contact force). To explore the role of externally applied bias in the patterning process, a number of bias voltages ranging from $5 \mathrm{~V}$ to $5 \mathrm{~V}$ were applied. The AFM tip was immersed into the acidic phosphate buffer solution of $\mathrm{pH}$ 4.0. During the patterning operation, the inked tip was scanned across the polymer surface at a writing speed of $80 \mathrm{~nm} / \mathrm{s}$ while the contact force (e.g., $1 \mu \mathrm{N}$ ) between the tip and surface was held constant. When no bias voltage was applied (Fig. 6.3.1a), the pattern formation was neither noticeable nor reproducible. When the tip was negatively biased (Fig. 6.3.1b), no structure was present. By contrast, swollen patterns consisting of continuous lines were obtained when the tip was positively biased (Fig. 6.3.1c). Topographic images in Fig. 6.3.2 show that the heights of the selected features are $13 \mathrm{~nm}$ (Fig. 6.3.2a), $6 \mathrm{~nm}$ (Fig. 6.3.2b) and $3 \mathrm{~nm}$ (Fig. 6.3.2c), corresponding to the applied bias voltages of $5 \mathrm{~V}, 3 \mathrm{~V}$ and $0 \mathrm{~V}$, respectively. To test the influence of tipsubstrate contact force on the pattern formation, experiments similar to the one described above were repeated using a fixed bias voltage (e.g., $5 \mathrm{~V}$ ) and varied contact forces ranging from $0.5 \mu \mathrm{N}$ to $1 \mu \mathrm{N}$. As shown in Fig. 6.3.2, the heights of the selected features are $8 \mathrm{~nm}$ (Fig. 6.3.2d) and $3 \mathrm{~nm}$ (Fig. 6.3.2e), corresponding to the contact forces of 0.8 $\mu \mathrm{N}$ and $0.5 \mu \mathrm{N}$, respectively. When the patterning operation was attempted using an uncoated tip (Fig. 6.3.1d), swollen pattern could not be obtained even if the tip was positively biased. 

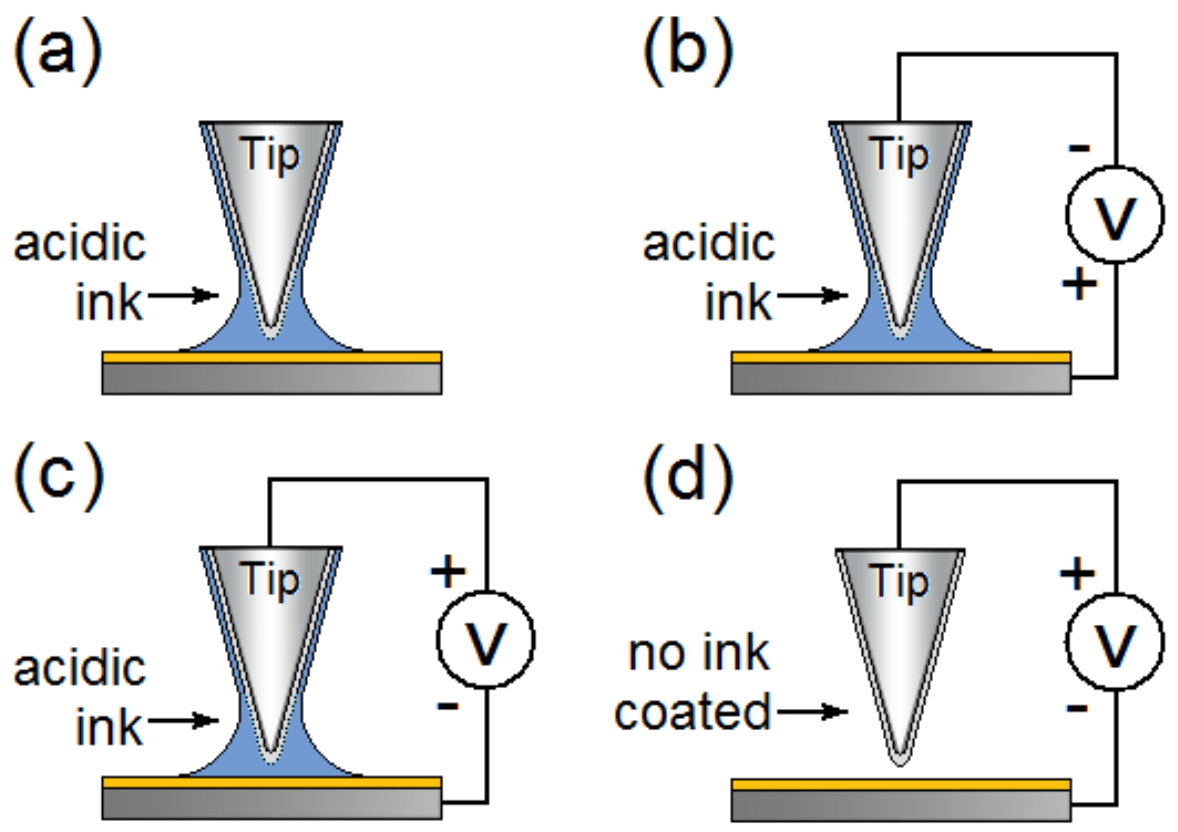

Fig. 6.3.1 Different experimental settings used for patterning features on P4VP polymer films: A tip was inked with the acidic buffer and (a) not biased, (b) negatively biased, (c) positively biased. (d) An uncoated tip while applying a positive bias.

This technique was also able to produce features with sub-100 nm width and complex patterns. A line feature with width down to $60 \mathrm{~nm}$ was created (Fig. 6.3.2f), along with a pattern of the letters: PSU (Fig. 6.3.2g), in a $5 \mu \mathrm{m} \times 5 \mu \mathrm{m}$ square region. In the latter, the average line width is about $180 \mathrm{~nm}$. 

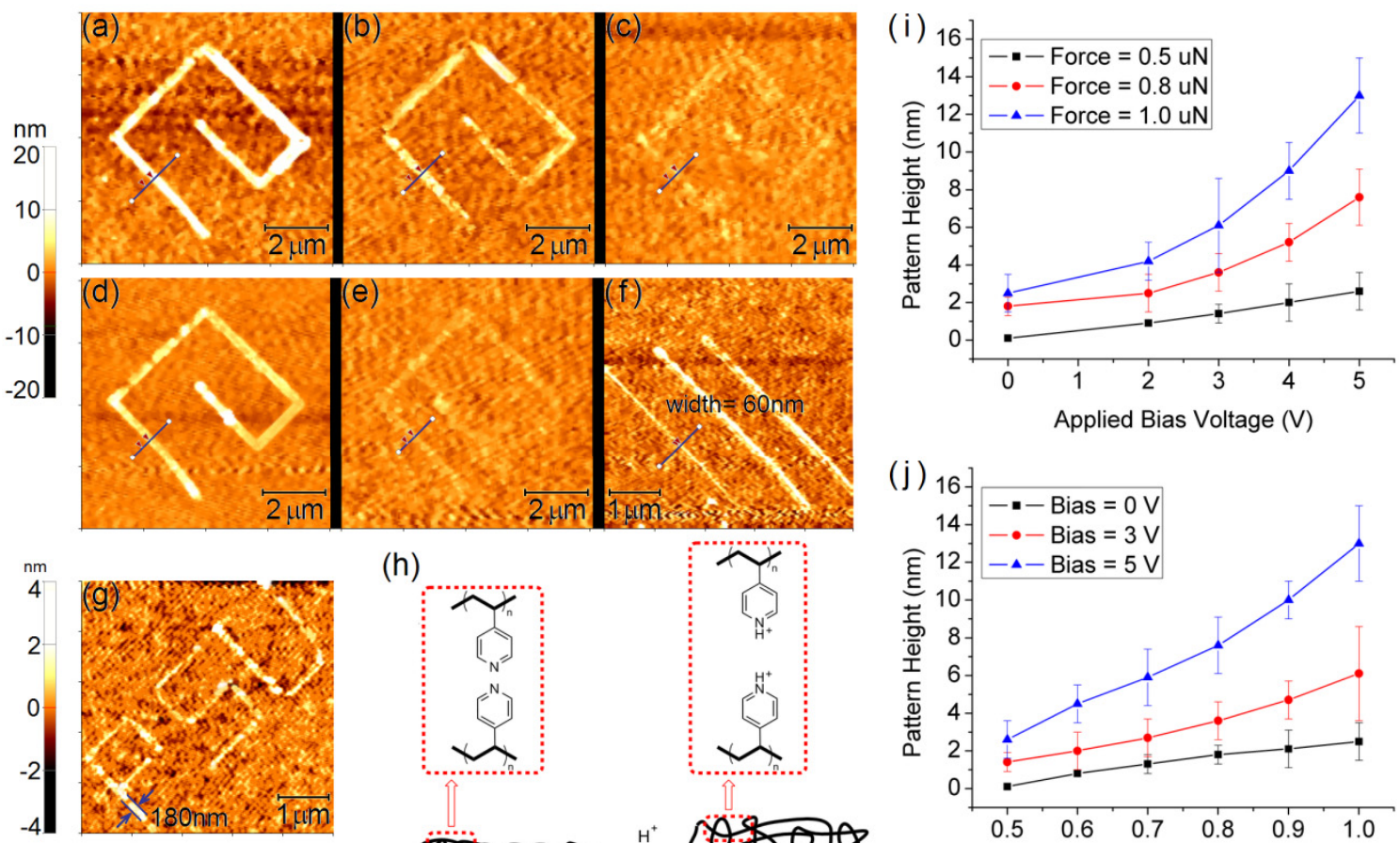

(h)

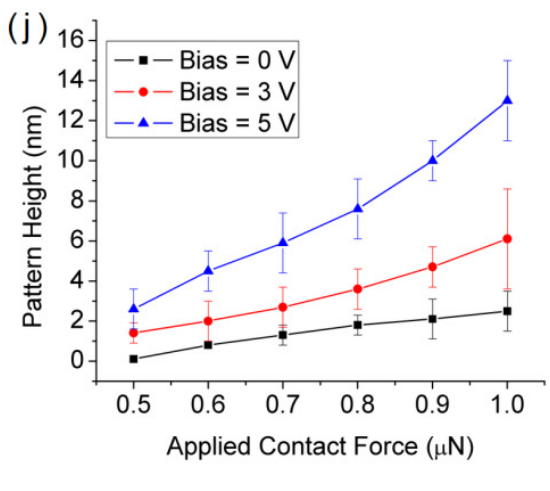

Fig. 6.3.2 (a-e) Topographic images of features drawn using the tip coated with acidic ink. The applied bias voltages $(\mathrm{V})$, contact forces $(\mu \mathrm{N})$ and measured heights of selected lines (nm) are the following: (a) 5, 1, 13; (b) 3, 1, 6; (c) 0, 1, 3; (d) 5, 0.8, 8; (e) 5, 0.5, 3. (f) A line feature with $60 \mathrm{~nm}$ wide. (g) A pattern of the letters: PSU. Image sizes: $8 \mu \mathrm{m} \times 8 \mu \mathrm{m}$ for (a-e); $5 \mu \mathrm{m} \times 5 \mu \mathrm{m}$ for (f-g). (h) Schematic showing that the swelling of P4VP film originates from the protonation of the pyridine units. (i) The pattern height v.s. bias voltages at various fixed contact forces. (j) The pattern height v.s. contact forces under different constant bias voltages.

To further support the claim that the presence of raised patterns was resulted from the swelling response of the P4VP polymer film, ${ }^{[59,64]}$ reversibility of the patterning process was demonstrated by selectively erasing an existing swollen pattern. "Dot" features were initially generated using a tip coated with acidic ink. Only the dot at the center of Fig. 6.3.3 (denoted by the dashed-line square) was then twice subjected to the "erasing" operation, which consisted of using the same tip but inked with a basic phosphate buffer solution of $\mathrm{pH} 8.3$ without applying the bias voltage. The removal of the existing dot was performed by keeping the tip in contact and stationary on top of the dot 
(no scanning movement). The images in Fig. 6.3.3 show the progressive attenuation of the dot height, from $12 \mathrm{~nm}$ to $7 \mathrm{~nm}$ and finally to $3 \mathrm{~nm}$. During the "erasing" process, the applied load exerted by the tip was kept intentionally low (i.e., $0.5 \mu \mathrm{N}$ ) so as to minimize potential damages to the dot pattern. Indeed, there was no change in the feature height when the "erasing" experiment was attempted using an uncoated tip and $0.5 \mu \mathrm{N}$ load. Altogether, this result indicates that the disappearance of the central dot in Fig. 6.3.3 was not due to mechanical damages caused by the tip but the presence of basic buffer solution.

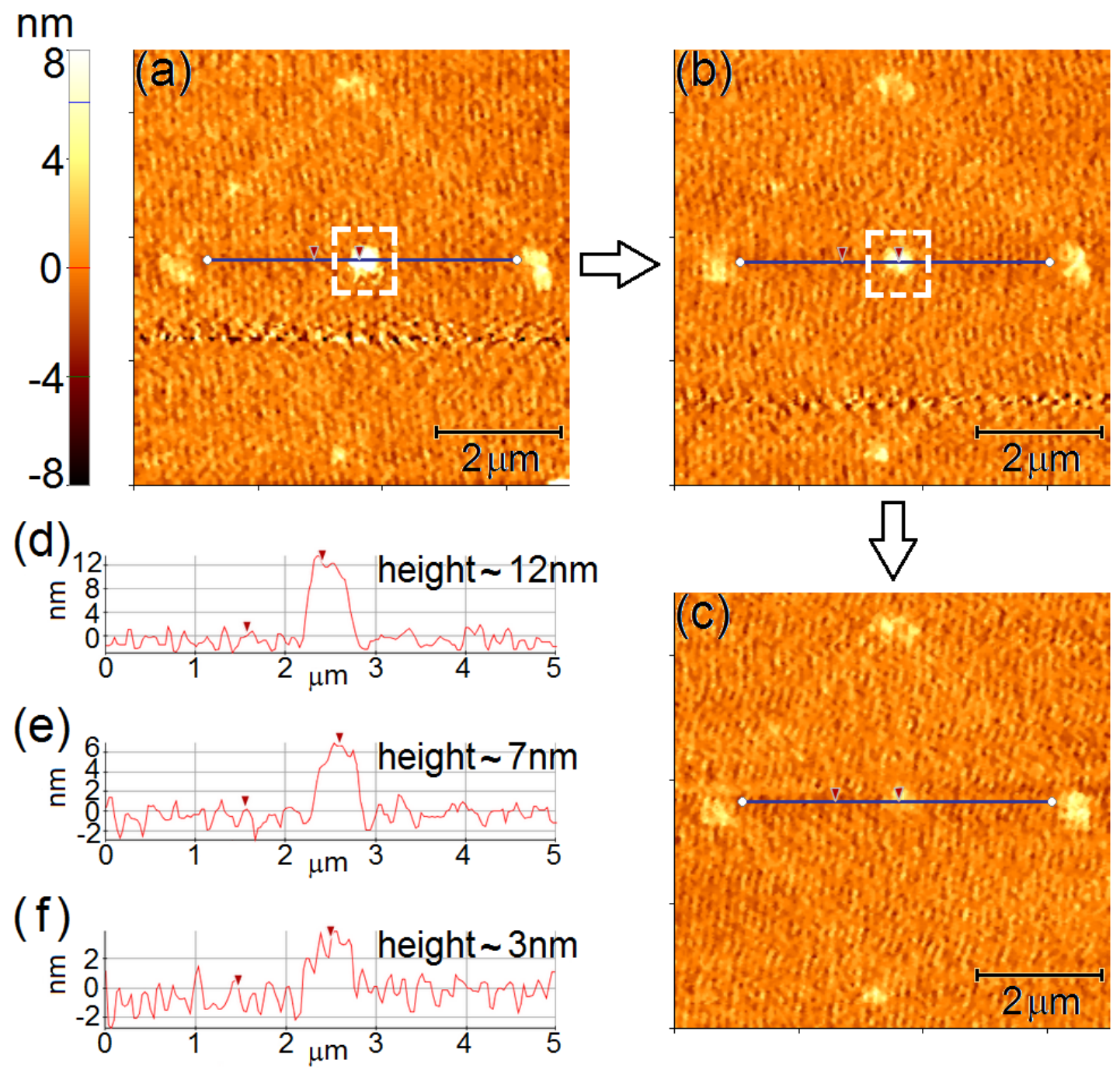

Fig. 6.3.3 Topographic images show the removal of the dot pattern at the center: (a) Initially, the dot height was $12 \mathrm{~nm}$; (b) After the first erasing performance, the height decreased to $7 \mathrm{~nm}$; (c) After the second erasing performance, the remaining height was 
only $3 \mathrm{~nm}$. Line profiles of (d), (e), and (f) correspond to the features selected in (a), (b), and (c), respectively.

Experimental results presented herein suggest that the essence of pattern formation on P4VP film is the local protonation of pyridine units of the polymer with hydronium ions $\left(\mathrm{H}_{3} \mathrm{O}^{+}\right)$. As outlined in Fig. 6.3.2h, the abundant acidic ions (i.e., $\mathrm{H}_{3} \mathrm{O}^{+}$) in the acidic ink delivered into the film cause the protonation of pyridine groups, yielding the pyridinium groups whose Coulomb repulsions between the charged centers make the polymer swell. Moreover, the attenuation of existing feature's height with subsequent deposition of the basic ink reveals that the swollen patterns are generated by polymer protonation rather than residual salt accumulation from the buffer solution. This observation provides the evidence on the reversible characteristic of the patterning process as well.

A conventional DPN process involves two steps: (1) the ink molecules transport from the coated tip to the substrate though the water meniscus that naturally forms between them, and (2) ink is adsorbed onto the surface to form a uniform monolayer. In our experiments, two major factors in controlling the properties of scribed patterns were found: the externally applied bias and tip-sample contact force. Comparison among the patterns in Fig. 6.3.2 indicates that higher bias voltages applied between the tip and sample yield lines with increasing heights at a fixed contact force $(1 \mu \mathrm{N})$. The influence of applied bias on feature height was further verified at different contact forces. Plots shown in Fig. 6.3.2i present the same trend in the height increase along with ascending the external voltage. It is well-known that the electric field across the polymer film is correlated to the bias voltage. A significant increase in the pattern's height is observed as the applied field strength is increased, indicating that the efficiency of acidic ion transport 
is electric-field dependent. Owing to the presence of applied electric field, the electrostatic force acts as a driving force to facilitate the deposition of acidic ions. Furthermore, since the patterning experiments were carried out in ambient conditions and the tip was initially dried with nitrogen, the electric field was required to anchor acidic ions to the substrate, so that the diffusive transport could occur consistently. In addition, the influence of Joule-heating discussed in electrostatic nanolithography ${ }^{[65,66]}$ was taken into consideration. In the experiment presented above, no swollen pattern was obtained by using an uncoated tip when the tip was positively biased. This result indicates that the pattern formation cannot be attributed to the Joule heating alone. However, since the ion mobility is greatly enhanced when the temperature increased ${ }^{[67]}$ we cannot completely eliminate the potential effect of Joule-heating. It could also be considered as a way to facilitate the ion transport.

Although the electric field has been utilized in electrochemical dip-pen nanolithography (E-DPN) ${ }^{[68]}$ the role of electric field played in our approach differs from in E-DPN where metallic or semiconducting structures are fabricated via electrochemical reactions. By contrast, no electrochemical reaction is needed for fabricating patterns on P4VP film, the external electric field applied during the patterning process simply made the surface patterning more reliable and repeatable.

Similar dependence of pattern height on contact force was found in our experiments: higher features arose from stronger contact force, which contrasted to the no effect on contact force reported by Weeks et al.. ${ }^{[69]}$ The dependencies of pattern height on contact forces under different constant bias voltages are summarized in Fig. 6.3.2j. The increase in the contact force exerted on the tip could lead to a greater tip area in contact with the 
polymer. Alternatively, this could result in broadening of the liquid meniscus between the tip and surface. Therefore, the increase in pattern's height with increasing the contact force could be attributed to the wider tip-sample effective contact area, where a greater amount of acidic ions could be transported from the tip to the polymer.

The breakdown of polymer film did not take place under the applied electric fields (bias voltages are from $-5 \mathrm{~V}$ to $5 \mathrm{~V}$ ). Additionally, no effect of external bias voltage on the vertical position of the tip was observed by monitoring the deflection of the AFM cantilever. It reveals that the electric force induced by the bias voltage can be eliminated. The high spring constant of non-contact AFM cantilevers (compared to general contact AFM cantilevers) was of significance for precise control of contact force during the patterning process.

The factors that affect the dimensions of the patterned features can be attributed but not limited to a combination of several variables, such as the writing speed, ${ }^{[52,69,70]}$ the effects of temperature and humidity, ${ }^{[57,69,71]}$ and the geometry and wettability of the tip. ${ }^{[71]}$ The dimensions of dot features as a function of the dwell time and relative humidity has been demonstrated in our previous work. ${ }^{[64]}$ No structures could be created when the humidity was below $30 \%$. It indicates that the transfer of acidic ions takes place when sufficient liquid is present between the tip and the sample. Furthermore, using a sharp tip on which remaining liquids were removed just before the patterning process was critical in achieving finer features. 


\subsection{Conclusions}

In conclusion, we demonstrated the fabrication of nanostructures on cross-linked P4VP thin films based on DPN technique. The protonation of the pyridine units of P4VP responsive to the acidic ions delivered into the film leads to the swollen pattern formation. Furthermore, we reported that the application of an electric field between the tip and the sample provides a simple method to precisely control the patterning process. The acidic ions (i.e., $\mathrm{H}_{3} \mathrm{O}^{+}$) are reliably transferred from the ink-coated tip to the polymer-covered substrate under the applied electric field. The effects of patterning parameters, such as applied bias and contact force, were investigated. The results suggest that both a higher bias voltage and a stronger contact force result in a marked increase in the pattern's height. Significantly, we illustrated that the removal of existing structures on P4VP films can be achieved by taking advantage of the reversible property of pattern formation. 


\section{CHAPTER 7. FINAL CONCLUSIONS}

By integrating acoustic sensing with the shear-force microscopy, a versatile system is being developed by our research group, which is named shear-force/acoustic near-field microscope (SANM). This system allows detection for shear-force damping acting on the microscope's probe as well as acoustic signal reflecting the sample's response. This dual sensing capability offers more information with regard to the probe-sample interaction since the shear-force and acoustic signals are recorded simultaneously.

Shear-force mechanism nowadays has been widely used for the distance control in scanning probe microscopes. However, the origin of shear-force damping cannot be explained well by present theoretical and experimental studies. Herein, the shear-force interaction between probe and sample as a function of the probe-sample distance was investigated in our shear-force/acoustic microscope by performing experiments at ambient conditions. The probe is a tapered gold tip attached to a quartz tuning fork. Gold tips can be reproducibly and reliably obtained using electrochemical etching. The sample is a piece of silicon wafer after appropriate chemical treatments, such as cleaning and chemical functionalization of surface. Based on the approach/retraction measurements, we find that the interaction between the probe and sample is dissipative, conservative, and mediated by a contamination layer existent on top of the solid support. The correlation between the amplitude of tip oscillation and acoustic signal support this conclusion. Furthermore, influences of tip geometry, environmental humidity, water affinity of tip and sample surfaces on the probe-sample interaction are discussed. They provide a chance to have an insight into the role of fluid-like contamination layer played in the shear-force damping. On the other hand, effects of other forces, such as 
electrostatic forces, van der Waals forces and friction forces, on the probe-sample interaction cannot be ruled out completely. We believe that the acoustic measurements can provide more information than the tuning fork on the interface of contamination layer and solid surface, as well as properties of the bulk sample.

The importance of the presence of water layers is also displayed in nanolithography. By delivering the hydronium ions from an AFM tip to a P4VP thin film through the naturally formed liquid bridge between them, swollen patterns are fabricated on the polymer substrate due to the protonation mechanism. Moreover, the application of electric field makes the patterning process reliable and reproducible. 


\section{CHAPTER 8. FUTURE RECOMMENDATION}

It will be interesting to investigate the probe-sample interaction in well controlled conditions, including a vacuum environment. In that way, we could distinguish the effect

of the contamination layer from the influences caused by other forces. Furthermore, if capillary forces caused by the presence of water layer were eliminated (for example, when performing the experiment under vacuum conditions), it would be possible to use the shear-force/acoustic microscope to study chemical forces between two chemically functionalized groups.

It is worth adding component in our present system to measure the normal force acting on the probe. Thus, more quantitative analysis can be applied for better understanding the probe-sample interaction.

It is known that the water affinity of silicon surface can vary from hydrophobicity (pure silicon) to hydrophilicity (silicon with an oxide layer) during the oxidation process of silicon in air. Therefore, the influence of the chemical properties on the adsorbed contamination layer could also be tested on the silicon substrate, which is freshly etched for removing its native oxide layer. With the growth of the oxide layer on silicon, it could be possible to monitor the switching of the wettability of the surface by performing the approach/retraction operations.

The real mechanical response of tuning fork should be obtained associated with acoustic measurements using the sensor placed under the sample. Topographic images of samples taken by implanting the acoustic signal into the feedback control will be achieved in near future. 


\section{REFERENCES}

[1] G. Binnig, H. Rohrer, C. Gerber, E. Weibel, Appl. Phys. Lett. 1982, 40, 178.

[2] G. Binning, H. Rohrer, C. Gerber, E. Weibel, Phys. Rev. Lett. 1982, 49, 57.

[3] I. H. Musselman, P. E. Russell, J. Vac. Sci. Technol., A 1990, 8, 3558.

[4] J. P. Ibe, P. P. Bey, S. L. Brandow, R. A. Brizzolara, N. A. Burnham, D. P. Dilella, K. P. Lee, C. R. K. Marrian, R. J. Colton, J. Vac. Sci. Technol., A 1990, 8, 3570.

[5] R. Kaneko, S. Oguchi, Jpn. J. Appl. Phys. 1990, 29, 1854.

[6] G. Binnig, C. F. Quate, C. Gerber, Phys. Rev. Lett. 1986, 56, 930.

[7] R. Garcia, A. San Paulo, Phys. Rev. B 1999, 60, 4961.

[8] R. Perez, I. Stich, M. C. Payne, K. Terakura, Phys. Rev. B 1998, 58, 10835.

[9] G. Meyer, N. M. Amer, Appl. Phys. Lett. 1988, 53, 1045.

[10] P. K. Hansma, J. P. Cleveland, M. Radmacher, D. A. Walters, P. E. Hillner, M. Bezanilla, M. Fritz, D. Vie, H. G. Hansma, C. B. Prater, J. Massie, L. Fukunaga, J. Gurley, V. Elings, Appl. Phys. Lett. 1994, 64, 1738.

[11] M. D. Kirk, T. R. Albrecht, C. F. Quate, Rev. Sci. Instrum. 1988, 59, 833.

[12] E. Betzig, P. L. Finn, J. S. Weiner, Appl. Phys. Lett. 1992, 60, 2484.

[13] K. Karrai, R. D. Grober, Appl. Phys. Lett. 1995, 66, 1842.

[14] R. A. Lemons, C. F. Quate, Appl. Phys. Lett. 1974, 24, 163.

[15] X. Q. Cui, A. La Rosa, Appl. Phys. Lett. 2005, 87, 231907.

[16] A. La Rosa, X. Cui, J. McCollum, N. Li, R. Nordstrom, Rev. Sci. Instrum. 2005, $76,093707$.

[17] D. Myers, in Surfaces, Interfaces, and Colloids: principles and applications, Wiley-VCH New York, 1991, pp. 21. 
[18] D. Myers, in Surfaces, Interfaces, and Colloids: principles and applications, Wiley-VCH New York, 1991, pp. 89.

[19] D. Myers, in Surfaces, Interfaces, and Colloids: principles and applications, Wiley-VCH New York, 1991, pp. 143.

[20] D. Myers, in Surfaces, Interfaces, and Colloids: principles and applications, Wiley-VCH New York, 1991, pp. 406.

[21] K. L. Johnson, in Contact Mechanics Cambridge University Press, Cambridge, UK, 1985, pp. 84.

[22] K. L. Johnson, K. Kendall, A. D. Roberts, Proc. R. Soc. London A 1971, 324, 301.

[23] J. N. Israelachvili, in Intermolecular and Surface Forces, Academic Press, London, 1991, pp. 326.

[24] J. N. Israelachvili, in Intermolecular and Surface Forces, Academic Press, London, 1991, pp. 330.

[25] J. N. Israelachvili, in Intermolecular and Surface Forces, Academic Press, London, 1991, pp. 319.

[26] J. N. Israelachvili, in Intermolecular and Surface Forces, Academic Press, London, 1991, pp. 322.

[27] J. Israelachvili, R. Pashley, Nature 1982, 300, 341.

[28] B. Ren, G. Picardi, B. Pettinger, Rev. Sci. Instrum. 2004, 75, 837.

[29] W. A. Atia, C. C. Davis, Appl. Phys. Lett. 1997, 70, 405.

[30] A. G. T. Ruiter, J. A. Veerman, K. O. vanderWerf, N. F. vanHulst, Appl. Phys. Lett. 1997, 71, 28.

[31] V. E. Granstaff, S. J. Martin, J. Appl. Phys. 1994, 75, 1319. 
[32] T. R. Albrecht, P. Grutter, D. Horne, D. Rugar, J. Appl. Phys. 1991, 69, 668.

[33] S. Davy, M. Spajer, D. Courjon, Appl. Phys. Lett. 1998, 73, 2594.

[34] F. F. Froehlich, T. D. Milster, Appl. Phys. Lett. 1997, 70, 1500.

[35] J. K. Jang, G. C. Schatz, M. A. Ratner, J. Chem. Phys. 2004, 120, 1157.

[36] G. He, M. H. Muser, M. O. Robbins, Science 1999, 284, 1650.

[37] R. Brunner, O. Marti, O. Hollricher, J. Appl. Phys. 1999, 86, 7100.

[38] P. K. Wei, W. S. Fann, J. Appl. Phys. 2000, 87, 2561.

[39] N. A. Burnham, R. J. Colton, H. M. Pollock, Phys. Rev. Lett. 1992, 69, 144.

[40] M. Yan, B. Harnish, Adv. Mater. 2003, 15, 244.

[41] M. Yan, M. A. Bartlett, Nano Lett. 2002, 2, 275.

[42] Y. Sun, H. Mortensen, S. Schar, A. S. Lucier, Y. Miyahara, P. Grutter, Phys. Rev. B 2005, 71, 193407.

[43] I. I. Smolyaninov, W. A. Atia, S. Pilevar, C. C. Davis, Ultramicroscopy 1998, 71, 177.

[44] J. U. Schmidt, H. Bergander, L. M. Eng, J. Appl. Phys. 2000, 87, 3108.

[45] R. D. Piner, J. Zhu, F. Xu, S. H. Hong, C. A. Mirkin, Science 1999, 283, 661.

[46] K. B. Lee, S. J. Park, C. A. Mirkin, J. C. Smith, M. Mrksich, Science 2002, 295, 1702.

[47] J. H. Lim, C. A. Mirkin, Adv. Mater. 2002, 14, 1474.

[48] H. T. Wang, O. A. Nafday, J. R. Haaheim, E. Tevaarwerk, N. A. Amro, R. G.

Sanedrin, C. Y. Chang, F. Ren, S. J. Pearton, Appl. Phys. Lett. 2008, 93, 143105.

[49] B. W. Maynor, Y. Li, J. Liu, Langmuir 2001, 17, 2575. 
[50] L. M. Demers, D. S. Ginger, S. J. Park, Z. Li, S. W. Chung, C. A. Mirkin, Science 2002, 296, 1836.

[51] A. Ivanisevic, C. A. Mirkin, J. Am. Chem. Soc. 2001, 123, 7887.

[52] C. Y. Huang, G. Q. Jiang, R. Advincula, Macromolecules 2008, 41, 4661.

[53] B. W. Maynor, J. Y. Li, C. G. Lu, J. Liu, J. Am. Chem. Soc. 2004, 126, 6409.

[54] M. S. Anderson, Anal. Chem. 2005, 77, 2907.

[55] M. Yang, P. E. Sheehan, W. P. King, L. J. Whitman, J. Am. Chem. Soc. 2006, 128, 6774.

[56] Y. Cui, Q. Q. Wei, H. K. Park, C. M. Lieber, Science 2001, 293, 1289.

[57] S. Rozhok, R. Piner, C. A. Mirkin, J. Phys. Chem. B 2003, 107, 751.

[58] S. H. Hong, J. Zhu, C. A. Mirkin, Langmuir 1999, 15, 7897.

[59] B. Harnish, J. T. Robinson, Z. C. Pei, O. Ramstrom, M. D. Yan, Chem. Mater. 2005, 17, 4092 .

[60] T. K. Tam, M. Ornatska, M. Pita, S. Minko, E. Katz, J. Phys. Chem. C 2008, 112, 8438.

[61] M. Privman, T. K. Tam, M. Pita, E. Katz, J. Am. Chem. Soc. 2009, 131, 1314.

[62] E. Vaganova, G. Meshulam, Z. Kotler, M. Rozenberg, S. Yitzchaik, J. Fluorescence 2000, 10, 81.

[63] J. E. Sader, J. W. M. Chon, P. Mulvaney, Rev. Sci. Instrum. 1999, 70, 3967.

[64] C. Maedler, S. Chada, X. Cui, M. Taylor, M. Yan, A. La Rosa, J. Appl. Phys. 2008, 104, 014311.

[65] S. F. Lyuksyutov, P. B. Paramonov, S. Juhl, R. A. Vaia, Appl. Phys. Lett. 2003, 83,4405 . 
[66] S. F. Lyuksyutov, R. A. Vaia, P. B. Paramonov, S. Juhl, L. Waterhouse, R. M. Ralich, G. Sigalov, E. Sancaktar, Nat. Mater. 2003, 2, 468.

[67] N. Cho, S. Ryu, B. Kim, G. C. Schatz, S. H. Hong, J. Chem. Phys. 2006, 124, 024714.

[68] Y. Li, B. W. Maynor, J. Liu, J. Am. Chem. Soc. 2001, 123, 2105.

[69] B. L. Weeks, A. Noy, A. E. Miller, J. J. De Yoreo, Phys. Rev. Lett. 2002, 88, 255505.

[70] J. Y. Jang, S. H. Hong, G. C. Schatz, M. A. Ratner, J. Chem. Phys. 2001, 115, 2721.

[71] J. Y. Jang, G. C. Schatz, M. A. Ratner, J. Chem. Phys. 2002, 116, 3875. 